medRxiv preprint doi: https://doi.org/10.1101/2020.08.23.20180299; this version posted August 25, 2020. The copyright holder for this preprint (which was not certified by peer review) is the author/funder, who has granted medRxiv a license to display the preprint in perpetuity.

It is made available under a CC-BY-NC-ND 4.0 International license .

\title{
The health sector cost of different policy responses to COVID-19 in low- and middle- income countries
}

\author{
Sergio Torres-Rueda, Sedona Sweeney, Fiammetta Bozzani and Anna Vassall ${ }^{1}$
}

\begin{abstract}
Much attention has focussed in recent months on the impact that COVID-19 has on health sector capacity, including critical care bed capacity and resources such as personal protective equipment. However, much less attention has focussed on the overall cost to health sectors, including the full human resource costs and the health system costs to address the pandemic. Here we present estimates of the total costs of COVID-19 response in low- and middle-income countries for different scenarios of COVID-19 mitigation over a one year period. We find costs vary substantially by setting, but in some settings even mitigation scenarios place a substantial fiscal impact on the health system. We conclude that the choices facing many low- and middle- income countries, without further rapid emergency financial support, are stark, between fully funding an effective COVID-19 reponse or other core essential health services.
\end{abstract}

This is preliminary report that has not yet been peer reviewed. These estimates should not yet guide policy in specific countries nor be reported as established information. We are placing these in the public domain to inform those who are estimaing Covid costs in low- and middle- income countries about the methods and assumptions required; higlight the broad level of fiscal impact and to invite comments for others working in this field, prior to submission to peer review publication.

These estimates have been subjected to a detailed validation and error checking process internally. Nevertheless, given the dearth of data to inform Covid cost estimates at this time, our results depend on a range of assumptions. Comments and suggestions to improve this work are welcomed

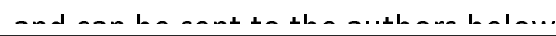

\footnotetext{
${ }^{1}$ All Centre for Health Economics in London (CHiL), London School of Hygiene and Tropical Medicine, Keppel Street, London WC1E 7HT. Comments addressed to: Sergio. TorresRueda@ Ishtm.ac.uk
} 
medRxiv preprint doi: https://doi.org/10.1101/2020.08.23.20180299; this version posted August 25, 2020. The copyright holder for this preprint (which was not certified by peer review) is the author/funder, who has granted medRxiv a license to display the preprint in perpetuity.

It is made available under a CC-BY-NC-ND 4.0 International license. 
medRxiv preprint doi: https://doi.org/10.1101/2020.08.23.20180299; this version posted August 25, 2020. The copyright holder for this preprint (which was not certified by peer review) is the author/funder, who has granted medRxiv a license to display the preprint in perpetuity.

It is made available under a CC-BY-NC-ND 4.0 International license .

\section{Introduction}

Since the declaration of Coronavirus Disease 2019 (COVID-19) as a public health emergency of international concern by the World Health Organization (WHO) in January 2020 [1], the disease has affected more than 22 million people and caused nearly 290,000 deaths worldwide [2]. While only a minority of cases will experience severe $(15 \%)$ or critical $(5 \%)$ disease that requires hospitalisation [3], the estimated costs of implementing WHO pandemic response guidelines in low- and middleincome countries (LMICS) are potentially substantial [4]. These estimates are all the more staggering in the context of over-stretched LMIC health systems, whose capacity to expand the provision of critical care was already limited before the COVID-19 pandemic $[5,6]$.

While there are financial costing tools available for countries to plan their short term resource requirements [7-9], there are few published estimates of the medium-term economic costs of prevention, tracing and treatment of COVID-19 cases for different epidemic scenarios to inform policy choice and longer term investment. Reliable economic cost estimates are essential for economic evaluations and priority setting around COVID-19 interventions. They can be used for example, to estimate potential cost savings from new COVID-19 vaccines as these become available' as well as for informing policy choices seeking to balance the broader macro-economic costs of mitigation strategies against the costs to the health system. Although to date, much of the policy concern rightly focuses on the sizeable health and macro-economic impact of COVID-19 in LMICS, it is important that the health sector costs of COVID-19 are also considered as the opportunity costs of the health sector response to COVID-19 on other health areas may be high $[10,11]$.

Most available cost data to inform these analyses is from high-income countries and/or does not encompass the full COVID-19 response cascade, nor the underlying health system costs [12-14]. To date, there is only one set of economic cost estimates from Kenya that takes into account low-cost case management scenarios appropriate to resource-constrained LMIC settings [15]. This paper presents the first study aiming to project the economic burden of COVID-19 response to LMIC health systems under different pandemic mitigation scenarios in the medium term. 


\section{Methods}

\section{Epidemiological scenarios}

Our estimates of COVID-19 cases for different scenarios come from the epidemiological model produced by the London School of Hygiene and Tropical Medicine, estimating the health impact of COVID-19 for 92 LMICs (https://cmmid.github.io/topics/covid19/LMIC-projection-reports.html). For each country, the model produces estimates on the number of cases, hospitalisations, number of days in hospital for severe cases (general ward) and critical cases (intensive care unit), and deaths for 57 distinct epidemiological scenarios over a one year period [16].

For the costing, four epidemiological scenarios were chosen out of the set of 57 possible scenarios. Scenario 1 represents an unmitigated epidemic. The other three scenarios were chosen because they represent a range of plausible policy options from highly mitigated to more moderate approaches (scenarios 4,14 and 49). Descriptions of the scenarios are presented below in Table 1. Further details can be found in the references above, and the numbers of cases, hospitalisations and bed-days for each country in Supplement S1.

\section{Defining the COVID-19 health sector response}

In line with the World Health Organisation we costed six priority areas of health sector response COVID-19: a) emergency response mechanisms at the national level; b) risk communication and community engagement; c) case finding, contact tracing and management; d) surveillance; e) public health measures; and, f) case management. For each of these we estimated a unit cost as per Table 2 below.

Our aim was to estimate a 'real world' cost that reflects local prices and resource use, and models of service delivery that are considered feasibile given resource constraints in low- and middle-income countries, rather than a full normative costing of national or global guidelines.

To estimate the activity level average (unit) costs for each of the above we used an ingredients based costing approach. Our basis for prices and resource use was recent local cost and resource use data from three countries: Ethiopia (low income country), Pakistan (lower middle income country, and South Africa (upper middle income country). We selected these countries as we were not able to collect primary cost data directly from COVID-19 service delivery points, so chose countries where we had recently conducted large scale costing exercises around either tuberculosis (TB) or general health services. This gave us current local data on actual resource use, input prices and health system unit cost data for services such as outpatient consultations, inpatient bed-days, a range of 
laboratory tests, including PCR tests and contact tracing. In the case of Ethiopia and South Africa, we had recent data from TB studies. In the case of Pakistan, the research team had been working with the Ministry of Health in 2019/20 to collect ingredients costs for all essential services as part of the Disease Control Priorities Project (DCP). While not primary data collection, all costs were subjected to a review by technical working groups as part of DCP that included practitioners at all levels of the health system. Members of the working groups were asked to consider feasibility as they reviewed and adapted the costs of different essential services.

To adapt this prior data on costs to COVID-19, we then used a combination of: review of COVID-19 resource planning tools and budgets, detailed expert consultation both with international and local experts and literature searches on primary cost data collection on the costs of clinical care in LMICs. Clinical management costs were based on expert consultation on essential critical care, including a detailed estimation of oxygen therapy needs; and considering recent cost data collection on critical care in $\mathrm{LMICS}^{20}$; and, length of stay and prevalence of complications from China. While we had access and reviewed length of stay data from different LMICs, this revealed either exceptionally high (early cases) or low (during surge) lengths of stay, and therefore the early data from China across the whole epidemic were felt to be the best estimate of a length of stay (considering those who die during care). Supplement S1 contains the summary level units used for each activity and the unit costs of each, and all assumptions used, including a comparison between normative recommendations for clinical case management and the assumptions used in this costing, based on expert consultation of feasibilty.

We then extrapolated the detailed ingredients costing done for Ethiopia, Pakistan and South Africa to low, lower middle, and upper middle income countries respectively. Each cost input in the ingredients costing was classified as a tradeable good, non-tradeable good, or staff cost. To convert the tradeable good from the base country (e.g. Ethiopia) to a 'second' country (e.g. Afghanistan) we apportioned the percentage of the unit cost that was composed of tradeable goods in 2019 US\$ from the base country to the second country.

Non-tradeable goods include buildings, heavy machinery, and other equipment. To convert these costs from a base country to a second country we used purchasing power parity (PPP) conversion rates. We multiplied the proportion of the unit cost that was defined as non-tradeable (in 2019 US\$) by the ratio of the 2019 GDP per capita (adjusted for PPP) of the second county, divided by the 2019 GDP per capita (adjusted for PPP) of the base country. Data on GDP per capita (adjusted for PPP) can be found in the World Bank database [17]. 
To convert staff costs from a base country to a second country we used conversion rates from Serje et al (2018) [18]. Serje et al (2018) use regression analysis on a dataset containing wages from health workers of different skill levels for 193 countries in order to predict wages by country income level relative to GDP per capita. We used the GDP per capita multipliers presented in the paper in order to convert the staff wages from the base country to the second country.

Finally, we then estimate the total cost of each scenario, against the \% of government and total health expenditure in each country and gross domestic product.

\section{Results}

Our combined primary data and expert reviewed ingreidents costs of managing a mild case with home visits from health care providers ranged from $\$ 13$ (Pakistan) to $\$ 147$ (South Africa) per case. Hospital-based care for a severe case ranged from $\$ 33.32$ (Pakistan) to $\$ 106$ (South Africa) per day, while costs for a critical case were much higher at \$221 (Pakistan) - \$1,082 (South Africa) per day in hospital (see supplmentary results appendix Table SR1). Table 2 shows our estimates of the mean unit cost per activity when these costs are extrapolated for low-income, lower-middle income, and upper-middle income countries. Table 2 includes the costs of managing the national emergency response mechanisms, risk communication and community engagement, case finding and surveillance, public health measures, screening and diagnosis, and case management.

Average cost per capita by country income category are reported in Table 3, considering the total number of cases, and other units. Table SR2 and SR3 show the underlying costs per capita and total estimated annual costs per country, by intervention scenario. For example, an unmitigated epidemic would cost an average of $\$ 5.2$ billion per country, ranging from $\$ 11.3$ million in Sao Tome and Principe to $\$ 127.8$ billion in India. This is equivalent to an average of $\$ 50$ per capita in LIC, $\$ 62$ in LMIC, and \$84 in UMIC (Table SR2). A 30-day lockdown would reduce these annual costs to an average of $\$ 4.66$ billion $(10.3 \mathrm{M}-115.1 \mathrm{~B})$, or $\$ 44-\$ 74 /$ capita. An intervention resulting in social distancing of $20 \%$ year-round would further reduce costs to 3.84 billion $(8.86 \mathrm{M}-96.08 \mathrm{~B})$, or $\$ 37$ $\$ 60 /$ capita. Finally, an intervention leading to generalized social distancing of $60 \%$ year-round would to costs that are on average less than half those of the unmitigated epidemic (5.15M-58.56B or \$2136 percapita).

In all scenarios, costs of the COVID-19 response were predominantly attributable to screening and diagnosis and case management, particularly management of critical cases (Table 4). Case finding, contact tracing and surveillance, and public health measures in contrast made up less than $5 \%$ of the 
medRxiv preprint doi: https://doi.org/10.1101/2020.08.23.20180299; this version posted August 25, 2020. The copyright holder for this preprint (which was not certified by peer review) is the author/funder, who has granted medRxiv a license to display the preprint in perpetuity.

It is made available under a CC-BY-NC-ND 4.0 International license .

total response costs. National-level costs of coordinating the emergency response and risk communication likewise constituted a small proportion of overall costs, amounting to less than $1 \%$ of total costs on average. The maps in Figures $1 \mathrm{a}$ and $1 \mathrm{~b}$ show the extent to which total costs increase with less stringent social distancing policies, and how social distancing can be more effective than temporary lockdown at containing costs.

Our sensitivity analysis (presented in Table SR8) shows that our estimates are partricularly sensitive to our assumptions on the number of symptomatic cases tested. Doubling the estimated number of symptomatic cases tested from $10 \%$ to $20 \%$ increased our 'unmitigated' cost estimates from $\$ 50$ $\$ 84 /$ capita to $\$ 57-\$ 90 /$ capita. A ten-fold increase in the number of symptomatic cases tested, from $10 \%$ to $100 \%$, resulted in nearly doubling our 'unmitigated' cost estimates to $\$ 121-137$ per capita. The effect of this assumption was constant across all scenarios.

\section{Discussion}

We find that the costs to health sector of responding to COVID-19 are substantial in LMICs, even when estimating the costs of essential critical care only. High levels of social distancing, however, may halve these costs within a one year period compared to allowing the pandemic to proceed in an unmitigated manner in the first year. The total cost and the costs as a proportion of health expenditue and GDP vary substantially across countries, with some countries likely to be able to 'afford' the costs, and for others the financial impact of COVID-19 on the health sector in its first year being higher than normal annual costs for the health sector in totality.

Our methods are subject to many limitations. Normally we would estimate 'real world' costs collecting extensive primary cost data on actual service delivery. In the case of COVID-19, we have not been able to do this. We have therefore had to rely substantially on data collected for other purposes and on expert opinion from LMICs to make key assumptions on how services may be delivered. We aimed to include the total costs to the health system and our aim was to estimate 'real world costs' in terms of the resource needs to deliver the most essential care. However, our costs are unlikely to reflect actual expenditures, as countries may either provide more care to specific patients, or struggle to provide even the most essential care given the current restrictions on expenditure. Likewise, the case numbers that our estimates rely upon are unlikely to match the real case numbers currently being observed in many LMICs, as they represent single policy options, and a time period of one year from the start of the epidemic. Finally, we do not include the costs of protecting health care workers delivering other essential services outside the COVID-19 response. 
medRxiv preprint doi: https://doi.org/10.1101/2020.08.23.20180299; this version posted August 25, 2020. The copyright holder for this preprint (which was not certified by peer review) is the author/funder, who has granted medRxiv a license to display the preprint in perpetuity.

It is made available under a CC-BY-NC-ND 4.0 International license.

Despite these limitations, our work highlights several critical qualitative recommendations for those working in COVID-19 policy. First, it is imperative, that global agencies and funders continue to act to ensure sufficient resources are made available globally for LMICS to respond, as the epidemic evolves. While much of the focus is on the macro-economic impact of COVID-19 and mortality impact, the fiscal impact on the health sector is likely to be substantial also needs to be considered if health systems in LMICs are to continue to deliver health services effectively. Second, it is clear that some countries are much more vulnerable to fiscal impact on the health sector than others. While specific short term financing needs for COVID-19 will fluctuate considerably over time, we highlight that some countries fundamentally will struggle to cope with almost any COVID-19 scenario, whereas others may be less vulnerable; which could help target support. Finally, our results demonstrate the myriad decisions about care, protection and patient experience that are required to plan resource needs of the response for which there is little discussion or data on what is feasible in LMICs. This is a task that cannot be met using a global perspective, but need country specific input to reflect the specific health system characteristics of each country. We therefore also call for urgent support to encourage interaction of economists, planners, service managers and epidemiological modellers to inform COVID-19 policy at the country level accross LMICs. 
medRxiv preprint doi: https://doi.org/10.1101/2020.08.23.20180299; this version posted August 25, 2020. The copyright holder for this preprint (which was not certified by peer review) is the author/funder, who has granted medRxiv a license to display the preprint in perpetuity.

It is made available under a CC-BY-NC-ND 4.0 International license .

\section{References}

1. WHO. Fact Sheet 15A. Addendum to Fact Sheet 15 on National Implementation Measures for the International Health Regulations 2005 (IHR). COVID-19 as a Public Health Emergency of International Concern (PHEIC) under the IHR. May 2020; Available from: https://extranet.who.int/sph/sites/default/files/documentlibrary/document/FS15A IHR COVID19 EN MAY 2020.pdf.

2. Dong, E., H. Du, and L. Gardner, An interactive web-based dashboard to track COVID-19 in real time. Lancet Infect Dis, 2020. 20(5): p. 533-534.

3. WHO. COVID-19 STRATEGY UPDATE. April 14 2020. Available from: https://www.who.int/docs/default-source/coronaviruse/covid-strategy-update14april2020.pdf.

4. Davies, N., et al. The impact of Coronavirus disease 2019 (COVID-19) on health systems and household resources in Africa and South Asia. 2020. DOI:

https://doi.org/10.1101/2020.05.06.20092734.

5. Baker, T., et al., Essential care of critical illness must not be forgotten in the COVID-19 pandemic. The Lancet, 2020.

6. Turner, H.C., et al., Achieving affordable critical care in low-income and middle-income countries. BMJ Glob Health, 2019. 4(3): p. e001675.

7. WHO. COVID-19 Essential Supplies Forecasting Tool. 29 April 2020. Available from: https://www.who.int/publications/m/item/covid-19-essential-supplies-forecasting-tool\#.

8. WHO. Emergency Global Supply Chain System (COVID-19) catalogue. August 142020. Available from: https://www.who.int/publications/i/item/emergency-global-supply-chainsystem-(covid-19)-catalogue.

9. WHO. Health Workforce Estimator (HWFE). May 15 2020. Available from: https://www.euro.who.int/en/health-topics/Health-systems/pages/strengthening-thehealth-system-response-to-covid-19/surge-planning-tools/health-workforce-estimatorhwfe.

10. Abbas, K., et al., Routine childhood immunisation during the COVID-19 pandemic in Africa: a benefit-risk analysis of health benefits versus excess risk of SARS-CoV-2 infection. Lancet Glob Health, 2020.

11. McQuaid, C.F., et al., The potential impact of COVID-19-related disruption on tuberculosis burden. The European respiratory journal, 2020: p. 2001718.

12. Gandjour, A. How much reserve capacity is justifiable for hospital pandemic preparedness? A cost-effectiveness analysis for COVID-19 in Germany. 2020. DOI:

https://doi.org/10.1101/2020.07.27.20162743.

13. Jin, H., et al. Estimating the cost-of-illness associated with the COVID-19 outbreak in China from January to March 2020. DOI: https://doi.org/10.1101/2020.05.15.20102863.

14. Li, X.Z., et al., Treatment of coronavirus disease 2019 in Shandong, China: a cost and affordability analysis. Infect Dis Poverty, 2020. 9(1): p. 78.

15. Barasa, E. and A. Kairu. What does it cost to treat a COVID-19 patient in Kenya? KEMRI Wellcome Trust Health Economics Research Unit, July 2020. . 21 August 2020]; Available from: https://kemri-wellcome.org/zp-content/uploads/2020/07/HERU-Policy-briefTreatment-costs-for-COVID-19-patients-in-Kenya.pdf.

16. Pearson, C., et al., Projections of COVID-19 epidemics in LMIC countries. 2020.

17. World Bank, World Bank Open Data. 2020.

18. Serje, J., et al., Global health worker salary estimates: an econometric analysis of global earnings data. Cost Eff Resour Alloc, 2018. 16: p. 10.

19. Cao, B., et al., A Trial of Lopinavir-Ritonavir in Adults Hospitalized with Severe Covid-19. N Engl J Med, 2020. 382 (19): p. 1787-1799. 
medRxiv preprint doi: https://doi.org/10.1101/2020.08.23.20180299; this version posted August 25, 2020. The copyright holder for this preprint (which was not certified by peer review) is the author/funder, who has granted medRxiv a license to display the preprint in perpetuity.

It is made available under a CC-BY-NC-ND 4.0 International license .

20. NHSDigital. Hospital admitted patient care activity 2018--19. [cited 29 March 2020]; Available from:

https://digital.nhs.uk/data-andinformation/publications/statistical/hospital-admitted-patien t-care-activity/2018-19.

21. Li, J. and e. al., Epidemiology of COVID-19: A Systematic Review and Meta-analysis of Clinical Characteristics, Risk factors and Outcomes. Journal of Medical Virology.

22. Srivastava, U., Anaesthesia gas supply: gas cylinders. Indian J Anaesth, 2013. 57(5): p. 500-6.

23. Afrox. Medical Oxygen. 2020 [cited 202010 July]; Available from: https://www.afroxshop.co.za/shop/en/za/medical-oxygen.

24. World Health Organization. Global Health Observatory (GHO) data. 27 March 2020]; Available from: http://www9.who.int/gho/en/.

25. Nishiura, H., N.M. Linton, and A.R. Akhmetzhanov Serial interval of novel coronavirus (2019nCoV) infections. 2020. DOI: https://doi.org/10.1101/2020.02.03.20019497.

26. Ministry of Health, Kenya. COVID-19 Outbreak in Kenya. Daily Situation Report-78. 3 June 202021 August 20020]; Available from: https://www.health.go.ke/wpcontent/uploads/2020/06/Kenya-COVID-19-SITREP-078-03-Jun-2020.pdf.

27. Government of South Africa. COVID-19 Corona Virus South African Resource Porta. 202021 August 2020]; Available from: https://sacoronavirus.co.za/.

28. Ministry of Health, Ethiopia. Ethiopia COVID 19 Monitoring Platform. 202021 August 2020]; Available from: http://www.moh.gov.et/ejcc/en/node/196.

29. Government of India. COVID Statewide Status. 21 August 2020]; Available from: https://www.mygov.in/corona-data/covid19-statewise-status/.

30. Pakistan, G.o. COVID19 Health Advisory Platform. 21 August 2020]; Available from: http://covid.gov.pk/.

31. WHO, WHO Global Health Expenditure Database. 


\section{Main Table and Figures}

Table 1 Scenario Descriptions

\begin{tabular}{|c|c|}
\hline Scenario 1 & Unmitigated epidemic \\
\hline Scenario 4 & $\begin{array}{l}\text { The whole population is covered in this intervention scenario. The intervention is } \\
\text { triggered by daily incidence reaching } 1 \text { per } 10,000 \text {. The intervention includes self- } \\
\text { isolation of symptomatic persons for duration of symptoms, modelled as an } \\
\text { additional reduction in contacts among symptomatic people of } 75 \% \text {. The } \\
\text { intervention includes distancing measures that reduce contacts at school by } 20 \\
\% \text {.The intervention includes distancing measures that reduce contacts at work by } \\
20 \% \text {.The intervention includes distancing measures that reduce contacts in other } \\
\text { settings by } 20 \% \text {. The intervention includes distancing measures that reduce } \\
\text { contacts in the home setting by } 0 \% \text {. There is no difference in intervention by age. }\end{array}$ \\
\hline Scenario 14 & $\begin{array}{l}\text { The whole population is covered in this intervention scenario. The intervention is } \\
\text { triggered by daily incidence reaching } 1 \text { per } 10,000 \text {. The intervention includes self- } \\
\text { isolation of symptomatic persons for duration of symptoms, modelled as an } \\
\text { additional reduction in contacts among symptomatic people of } 25 \% \text {. The } \\
\text { intervention includes distancing measures that reduce contacts at school by } 60 \\
\% \text {.The intervention includes distancing measures that reduce contacts at work by } \\
60 \% \text {. The intervention includes distancing measures that reduce contacts in other } \\
\text { settings by } 60 \% \text {. The intervention includes distancing measures that reduce } \\
\text { contacts in the home setting by } 0 \% \text {. There is no difference in intervention by age. }\end{array}$ \\
\hline Scenario 49 & $\begin{array}{l}\text { 49. The intervention is temporary lockdown which leads to } 100 \% \text { of the } \\
\text { population reducing their contacts through school, home, work and other settings } \\
\text { by } 100 \%, 0 \%, 37.5 \% \text { and } 37.5 \% \text { respectively for whilst in lockdown. Lockdown } \\
\text { occurs for the first } 30 \text { days. After lockdown is lifted } 100 \% \text { of the population } \\
\text { reducing their contacts through school, home, work and other settings by } 20 \%, 0 \\
\%, 20 \% \text { and } 20 \% \text { respectively for whilst not in lockdown }\end{array}$ \\
\hline
\end{tabular}


Table 2. Weighted average (mean) unit cost per activity by country income category (2019 US\$)

\begin{tabular}{|l|r|r|r|}
\hline & Low Income & \multicolumn{1}{|c|}{$\begin{array}{c}\text { Lower-Middle } \\
\text { Income } \\
\text { Countries } \\
\text { (LMIC) }\end{array}$} & \multicolumn{1}{|c|}{$\begin{array}{c}\text { Upper-Middle } \\
\text { Income } \\
\text { Countries } \\
\text { (UMIC) }\end{array}$} \\
\hline $\begin{array}{l}\text { 1.a. Emergency Response Mechanisms: } \\
\text { National level }\end{array}$ & $1,150.10$ & $2,697.74$ & $6,243.97$ \\
\hline $\begin{array}{l}\text { 1.b. Emergency Response Mechanisms: } \\
\text { Training of health staff }\end{array}$ & $7,917.32$ & $17,602.46$ & $44,470.55$ \\
\hline $\begin{array}{l}\text { 2. } \text { Risk communication \& community } \\
\text { engagement }\end{array}$ & 101.44 & 240.58 & 551.17 \\
\hline $\begin{array}{l}\text { 3.a. Case finding, contact tracing and } \\
\text { management: Contact tracing }\end{array}$ & 2.93 & 9.84 & 19.24 \\
\hline $\begin{array}{l}\text { 3.b. Case finding, contact tracing and } \\
\text { management: Quarantine of contacts }\end{array}$ & 2.83 & 6.36 & 15.81 \\
\hline 4.a. Surveillance: Case notification & 2.83 & 6.36 & 15.81 \\
\hline 4.b. Surveillance: Reporting (national level) & 6.99 & 14.89 & 40.47 \\
\hline $\begin{array}{l}\text { 5. Public health measures: Hygiene } \\
\text { education }\end{array}$ & 61.29 & 145.82 & 333.91 \\
\hline 6. Screening and diagnosis & 30.86 & 37.85 & 63.98 \\
\hline 7.a. Case Management: Home-based care & 17.56 & 51.53 & 202.55 \\
\hline $\begin{array}{l}\text { 7.b. Case Management: Hospital-based } \\
\text { (severe case) }\end{array}$ & 34.74 & 42.68 & 136.44 \\
\hline $\begin{array}{l}\text { 7.c. Case Management: Hospital-based } \\
\text { (critical case) }\end{array}$ & 299.28 & 329.74 & $1,379.74$ \\
\hline 7.d. Case Management: Death & 64.52 & 64.52 & 64.52 \\
\hline
\end{tabular}

Table 3. Average Cost per Capita by Country Income Category (2019 US\$)

\begin{tabular}{|l|r|r|r|r|}
\hline \multirow{2}{*}{} & \multicolumn{4}{|l|}{$\begin{array}{l}\text { Average Cost per Capita by Country Income Category } \\
\text { (2019 US\$) }\end{array}$} \\
\cline { 2 - 5 } & Scenario 1 & Scenario 4 & Scenario 14 & Scenario 49 \\
\hline Low Income Countries (LIC) & 49.21 & 36.73 & 20.52 & 43.60 \\
\hline $\begin{array}{l}\text { Lower-Middle Income Countries } \\
\text { (LMIC) }\end{array}$ & 61.14 & 45.96 & 27.34 & 54.98 \\
\hline $\begin{array}{l}\text { Upper-Middle Income Countries } \\
\text { (UMIC) }\end{array}$ & 82.87 & 59.02 & 35.49 & 72.84 \\
\hline
\end{tabular}


Table 4. Average \% of Total Costs by Activity by Country Income Category by Scenario

\begin{tabular}{|c|c|c|c|c|c|c|c|c|c|c|c|c|}
\hline & \multicolumn{3}{|c|}{ Scenario 1} & \multicolumn{3}{|c|}{ Scenario 4} & \multicolumn{3}{|c|}{ Scenario 14} & \multicolumn{3}{|c|}{ Scenario 49} \\
\hline & LIC & LMIC & UMIC & LIC & LMIC & UMIC & LIC & LMIC & UMIC & LIC & LMIC & UMIC \\
\hline $\begin{array}{l}\text { 1.a. Emergency Response } \\
\text { Mechanisms: National level }\end{array}$ & $0.03 \%$ & $0.01 \%$ & $0.02 \%$ & $0.04 \%$ & $0.02 \%$ & $0.02 \%$ & $0.07 \%$ & $0.03 \%$ & $0.04 \%$ & $0.03 \%$ & $0.01 \%$ & $0.02 \%=$ \\
\hline $\begin{array}{l}\text { 1.b. Emergency Response } \\
\text { Mechanisms: Training of health } \\
\text { staff }\end{array}$ & $0.05 \%$ & $0.12 \%$ & $0.18 \%$ & $0.07 \%$ & $0.16 \%$ & $0.27 \%$ & $0.13 \%$ & $0.27 \%$ & $0.44 \%$ & $0.06 \%$ & $0.13 \%$ & 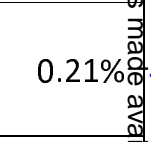 \\
\hline $\begin{array}{l}\text { 2. Risk communication } \& \\
\text { community engagement }\end{array}$ & $0.00 \%$ & $0.00 \%$ & $0.00 \%$ & $0.00 \%$ & $0.00 \%$ & $0.00 \%$ & $0.01 \%$ & $0.00 \%$ & $0.00 \%$ & $0.00 \%$ & $0.00 \%$ & $0.00 \% \frac{\overline{0}}{\frac{\overline{0}}{(1)}}$ \\
\hline $\begin{array}{l}\text { 3.a. Case finding, contact tracing } \\
\text { and management: Contact tracing }\end{array}$ & $0.96 \%$ & $2.19 \%$ & $1.24 \%$ & $0.98 \%$ & $2.27 \%$ & $1.31 \%$ & $0.96 \%$ & $2.23 \%$ & $1.25 \%$ & $0.96 \%$ & $2.22 \%$ & $1.25 \% \frac{\overline{ }}{\mathrm{\Phi}}$ \\
\hline $\begin{array}{l}\text { 3.b. Case finding, contact tracing } \\
\text { and management: Quarantine of } \\
\text { contacts }\end{array}$ & $0.93 \%$ & $1.17 \%$ & $1.04 \%$ & $0.95 \%$ & $1.21 \%$ & $1.10 \%$ & $0.93 \%$ & $1.17 \%$ & $1.05 \%$ & $0.93 \%$ & $1.18 \%$ & $1.06 \% \frac{\text { ค }}{\text { 苗 }}$ \\
\hline 4.a. Surveillance: Case notification & $0.13 \%$ & $0.17 \%$ & $0.15 \%$ & $0.14 \%$ & $0.17 \%$ & $0.16 \%$ & $0.13 \%$ & $0.17 \%$ & $0.15 \%$ & $0.13 \%$ & $0.17 \%$ & 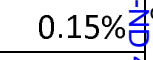 \\
\hline $\begin{array}{l}\text { 4.b. Surveillance: Reporting } \\
\text { (national level) }\end{array}$ & $0.00 \%$ & $0.00 \%$ & $0.01 \%$ & $0.00 \%$ & $0.01 \%$ & $0.01 \%$ & $0.01 \%$ & $0.01 \%$ & $0.02 \%$ & $0.00 \%$ & $0.01 \%$ & $0.01 \% \frac{\stackrel{A}{0}}{\frac{\vec{D}}{\vec{\oplus}}}$ \\
\hline $\begin{array}{l}\text { 5. Public health measures: } \\
\text { Hygiene education }\end{array}$ & $0.00 \%$ & $0.00 \%$ & $0.00 \%$ & $0.00 \%$ & $0.00 \%$ & $0.00 \%$ & $0.00 \%$ & $0.00 \%$ & $0.00 \%$ & $0.00 \%$ & $0.00 \%$ & $0.00 \% \frac{\text { a }}{\bar{y}}$ \\
\hline 6. Screening and diagnosis & \multirow{2}{*}{$\begin{array}{r}58.42 \% \\
1.19 \% \\
\end{array}$} & \multirow{2}{*}{$\begin{array}{r}48.42 \% \\
2.37 \%\end{array}$} & \multirow{2}{*}{$\begin{array}{r}25.10 \% \\
2.56 \%\end{array}$} & $59.71 \%$ & $50.07 \%$ & $26.54 \%$ & \multirow{2}{*}{$\begin{array}{r}58.25 \% \\
1.19 \%\end{array}$} & \multirow{2}{*}{$\begin{array}{r}48.58 \% \\
2.40 \%\end{array}$} & \multirow{2}{*}{$\begin{array}{r}25.26 \% \\
2.59 \%\end{array}$} & $58.78 \%$ & $48.91 \%$ & 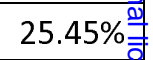 \\
\hline $\begin{array}{l}\text { 7.a. Case Management: Home- } \\
\text { based care }\end{array}$ & & & & $1.22 \%$ & $2.45 \%$ & $2.72 \%$ & & & & $1.20 \%$ & $2.39 \%$ & 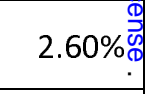 \\
\hline $\begin{array}{l}\text { 7.b. Case Management: Hospital- } \\
\text { based (severe case) }\end{array}$ & $6.65 \%$ & $8.50 \%$ & $10.72 \%$ & $6.41 \%$ & $8.14 \%$ & $10.44 \%$ & $6.67 \%$ & $8.40 \%$ & $10.67 \%$ & $6.58 \%$ & $8.39 \%$ & $10.65 \%$ \\
\hline $\begin{array}{l}\text { 7.c. Case Management: Hospital- } \\
\text { based (critical case) }\end{array}$ & $31.23 \%$ & $36.62 \%$ & $58.79 \%$ & $30.10 \%$ & $35.10 \%$ & $57.25 \%$ & $31.26 \%$ & $36.33 \%$ & $58.34 \%$ & $30.92 \%$ & $36.16 \%$ & $58.40 \%$ \\
\hline 7.d. Case Management: Death & $0.40 \%$ & $0.43 \%$ & $0.19 \%$ & $0.39 \%$ & $0.41 \%$ & $0.18 \%$ & $0.40 \%$ & $0.42 \%$ & $0.18 \%$ & $0.40 \%$ & $0.42 \%$ & $0.18 \%$ \\
\hline
\end{tabular}


medRxiv preprint doi: https://doi.org/10.1101/2020.08.23.20180299; this version posted August 25, 2020. The copyright holder for this preprint (which was not certified by peer review) is the author/funder, who has granted medRxiv a license to display the preprint in perpetuity.

\begin{abstract}
It is made available under a CC-BY-NC-ND 4.0 International license.
\end{abstract}
Figure 1a. Total costs as a proportion of GDP per capita

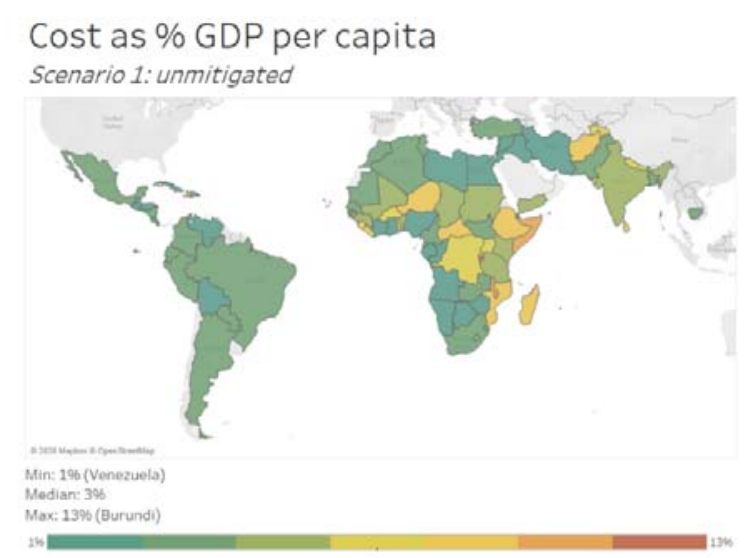

Cost as \% GDP per capita

Scenario 14:60\% distancing

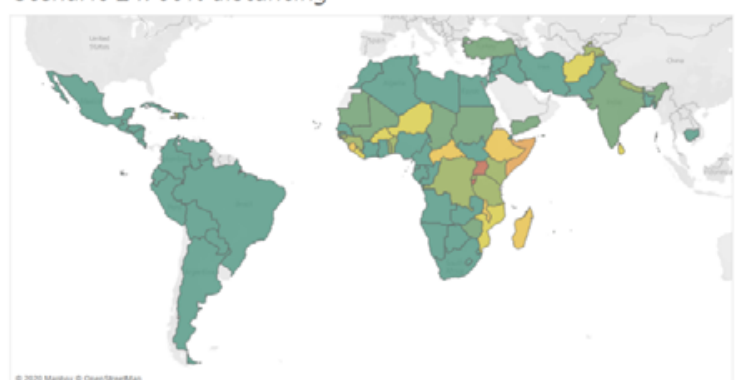

Min: $0.4 \%$ (Venezuels)

Median: 196

Max: 69s (Burundi)
Cost as \% GDP per capita

Scenario 4: $20 \%$ distancing

Min: 196 (Venezuela)

Median: 296

Bar: 109 (Burundi)

Cost as \% GDP per capita

Scenario 49: 30-day lockdown

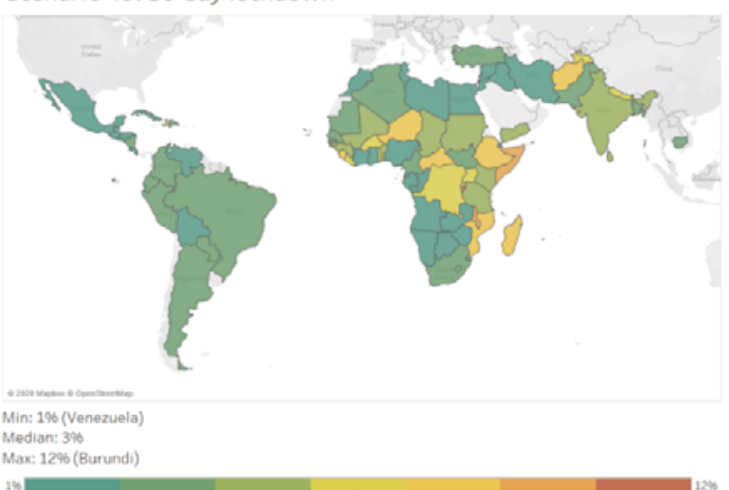


medRxiv preprint doi: https://doi.org/10.1101/2020.08.23.20180299; this version posted August 25, 2020. The copyright holder for this preprint (which was not certified by peer review) is the author/funder, who has granted medRxiv a license to display the preprint in perpetuity.

It is made available under a CC-BY-NC-ND 4.0 International license.

Figure $1 \mathrm{~b}$. Total costs as a proportion of total health spending, including out-of-pocket payments

Cost as \% total health spending (incl. OOP)

Scenario 1: unmitigated

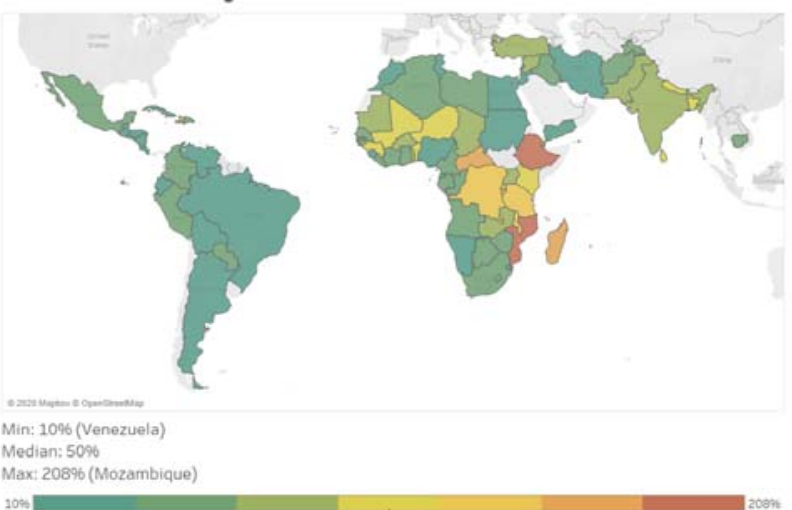

Cost as \% total health spending (incl. OOP) Scenario 4: $20 \%$ distancing

Cost as \% total health spending (incl. OOP) Scenario 14: $60 \%$ distancing
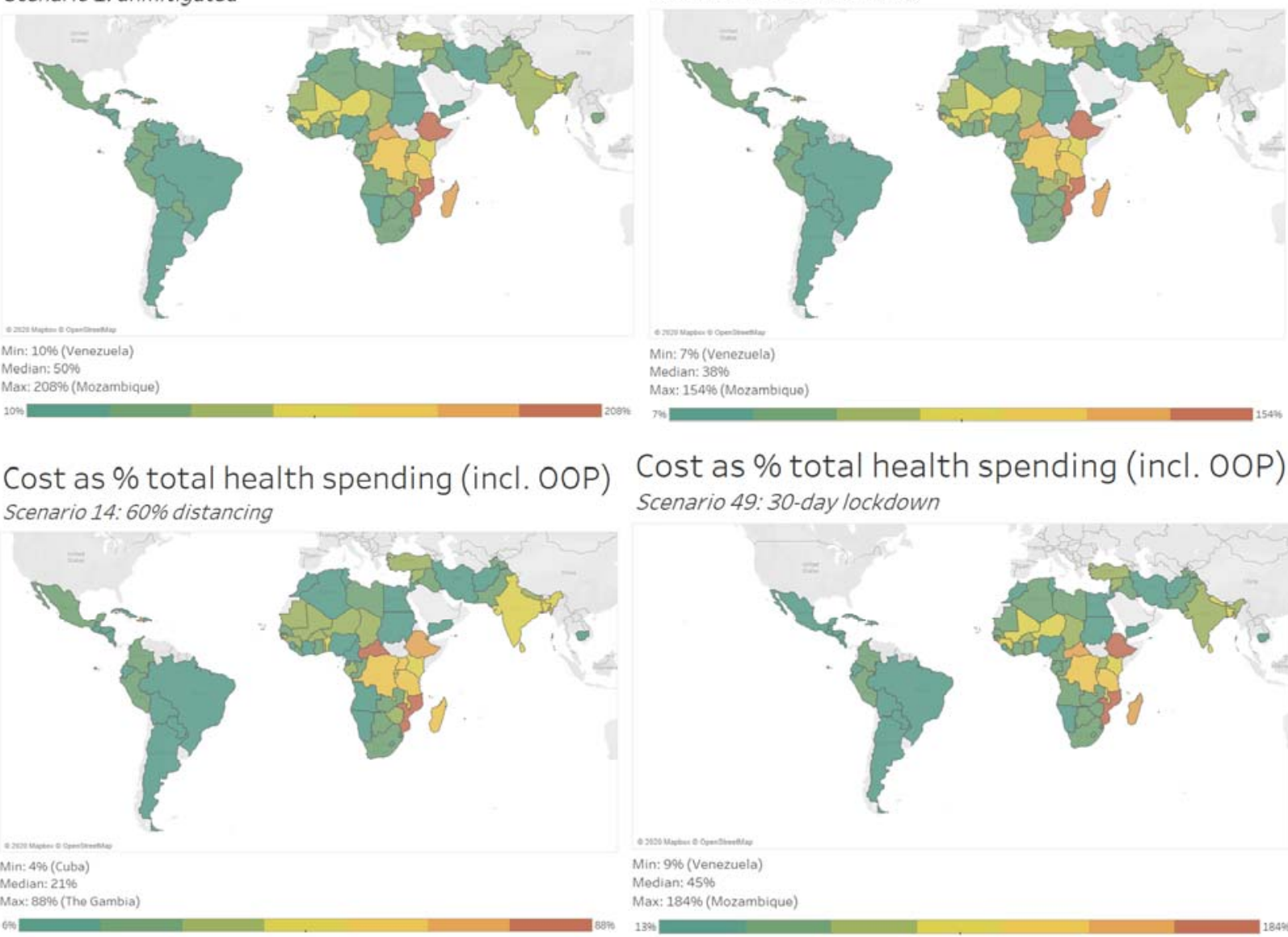

Cost as \% total health spending (incl. OOP) Scenario 49: 30-day lockdown
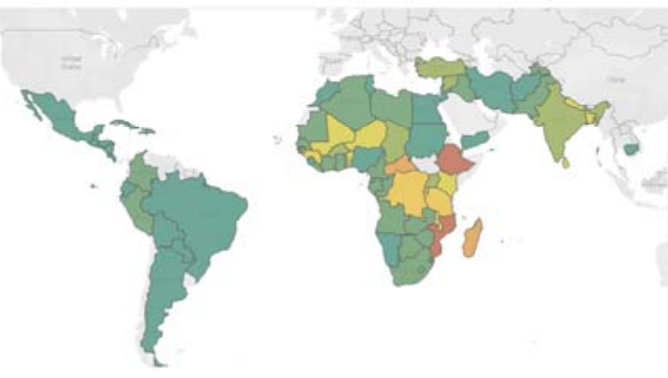

Max: 8896 (The Gambia) 
medRxiv preprint doi: https://doi.org/10.1101/2020.08.23.20180299; this version posted August 25, 2020. The copyright holder for this preprint (which was not certified by peer review) is the author/funder, who has granted medRxiv a license to display the preprint in perpetuity.

It is made available under a CC-BY-NC-ND 4.0 International license.

\section{Supplemental Results Tables}

Table SR1. Unit costs per activity for base countries (2019 US\$)

\begin{tabular}{|l|l|r|r|r|}
\hline & & \multicolumn{2}{|l|}{ Unit Cost (2019 US\$) } \\
\cline { 3 - 5 } Activity & Unit Type & $\begin{array}{l}\text { Ethio } \\
\text { pia }\end{array}$ & \multicolumn{1}{|l|}{$\begin{array}{l}\text { Pakist } \\
\text { an }\end{array}$} & $\begin{array}{l}\text { South } \\
\text { Africa }\end{array}$ \\
\hline 1.a. Emergency Response Mechanisms: National level & Per country per day & $\begin{array}{r}559.2 \\
6\end{array}$ & $\begin{array}{r}778.9 \\
0\end{array}$ & $\begin{array}{r}7,697.1 \\
6\end{array}$ \\
\hline $\begin{array}{l}\text { 1.b. Emergency Response Mechanisms: Training of } \\
\text { health staff }\end{array}$ & One-off per site & $\begin{array}{r}4,813 . \\
58\end{array}$ & $\begin{array}{r}8,096 . \\
53\end{array}$ & $\begin{array}{r}68,141 . \\
36\end{array}$ \\
\hline 2. Risk communication \& community engagement & Per country per day & 74.14 & 91.67 & $\begin{array}{r}1,133.4 \\
4\end{array}$ \\
\hline $\begin{array}{l}\text { 3.a. Case finding, contact tracing and management: } \\
\text { Contact tracing }\end{array}$ & Per person contacted & 3.48 & 2.54 & 26.23 \\
\hline $\begin{array}{l}\text { 3.b. Case finding, contact tracing and management: } \\
\text { Quarantine of contacts }\end{array}$ & Per person quarantined & 1.72 & 2.35 & 29.22 \\
\hline 4.a. Surveillance: Case notification & Per positive case & 1.72 & 2.35 & 29.22 \\
\hline 4.b. Surveillance: Reporting (national level) & Per country per week & 3.69 & 6.52 & 68.26 \\
\hline 5. Public health measures: Hygiene education & $\begin{array}{l}\text { Per education campaign per } \\
\text { month }\end{array}$ & 44.58 & 54.66 & 682.05 \\
\hline 6. Screening and diagnosis & $\begin{array}{l}\text { Per person screened and } \\
\text { tested }\end{array}$ & 36.97 & 26.98 & 73.12 \\
\hline 7.a. Case Management: Home-based care & $\begin{array}{l}\text { Per person requiring home- } \\
\text { based care }\end{array}$ & 22.90 & 12.45 & 146.57 \\
\hline 7.b. Case Management: Hospital-based (severe case) & $\begin{array}{l}\text { Per day of hospitalisation } \\
\text { (severe case) }\end{array}$ & 35.29 & 33.32 & 105.88 \\
\hline 7.c. Case Management: Hospital-based (critical case) & $\begin{array}{l}\text { Per day of hospitalisation } \\
\text { (critical case) }\end{array}$ & $\begin{array}{r}505.5 \\
6\end{array}$ & $\begin{array}{r}221.1 \\
8\end{array}$ & $\begin{array}{r}1,081.9 \\
4\end{array}$ \\
\hline 7.d. Case Management: Death & Per CovID-related death & 64.52 & 64.52 & 64.52 \\
\hline
\end{tabular}

Table SR2: Cost per Capita by Country per Scenario (2019 US\$)

\begin{tabular}{|c|c|c|c|c|c|c|}
\hline Country & & & & & Scenario 14 & Scenario 49 \\
\hline Afghanistan & $\$$ & 42.91 & $\$$ & 31.69 & $\begin{array}{l}\$ \\
17.89\end{array}$ & $\begin{array}{l}\$ \\
37.66\end{array}$ \\
\hline Algeria & $\$$ & 170.48 & $\$$ & 113.61 & $\begin{array}{l}\$ \\
59.33\end{array}$ & $\begin{array}{l}\$ \\
144.09\end{array}$ \\
\hline Angola & $\$$ & 55.30 & $\$$ & 43.35 & $\begin{array}{l}\$ \\
21.15\end{array}$ & $\begin{array}{l}\$ \\
50.86\end{array}$ \\
\hline Argentina & $\$$ & 381.86 & $\$$ & 272.68 & $\begin{array}{l}\$ \\
172.94\end{array}$ & $\begin{array}{l}\$ \\
337.16\end{array}$ \\
\hline Bangladesh & $\$$ & 60.48 & $\$$ & 45.53 & $\begin{array}{c}\$ \\
25.80\end{array}$ & $\begin{array}{c}\$ \\
54.30\end{array}$ \\
\hline Benin & $\$$ & 53.05 & $\$$ & 40.34 & $\begin{array}{l}\$ \\
21.95\end{array}$ & $\begin{array}{l}\$ \\
47.44\end{array}$ \\
\hline Bolivia & $\$$ & 73.01 & $\$$ & 53.55 & $\begin{array}{l}\$ \\
23.96\end{array}$ & $\begin{array}{l}\$ \\
65.06\end{array}$ \\
\hline Botswana & $\$$ & 186.86 & $\$$ & 134.47 & $\begin{array}{l}\$ \\
75.96\end{array}$ & $\begin{array}{l}\$ \\
163.93\end{array}$ \\
\hline Brazil & $\$$ & 272.86 & $\$$ & 191.02 & $\begin{array}{l}\$ \\
109.30\end{array}$ & $\begin{array}{c}\$ \\
237.90\end{array}$ \\
\hline Burkina Faso & $\$$ & 47.56 & $\$$ & 36.44 & $\begin{array}{l}\$ \\
21.13\end{array}$ & $\begin{array}{l}\$ \\
42.53\end{array}$ \\
\hline Burundi & $\$$ & 36.54 & $\$$ & 27.25 & $\begin{array}{l}\$ \\
14.96\end{array}$ & $\begin{array}{l}\$ \\
32.19\end{array}$ \\
\hline Cabo Verde & $\$$ & 72.18 & $\$$ & 51.79 & $\begin{array}{l}\$ \\
29.02\end{array}$ & $\begin{array}{l}\$ \\
63.29\end{array}$ \\
\hline Cambodia & $\$$ & 49.37 & $\$$ & 34.85 & $\begin{array}{l}\$ \\
14.09\end{array}$ & $\begin{array}{l}\$ \\
42.52\end{array}$ \\
\hline Cameroon & $\$$ & 45.79 & $\$$ & 35.08 & $\begin{array}{c}\$ \\
19.23\end{array}$ & $\begin{array}{c}\$ \\
41.36\end{array}$ \\
\hline Central African Republic & $\$$ & 39.10 & $\$$ & 29.37 & $\begin{array}{l}\$ \\
17.99\end{array}$ & $\begin{array}{l}\$ \\
34.60\end{array}$ \\
\hline Chad & $\$$ & 42.57 & $\$$ & 30.45 & $\begin{array}{c}\$ \\
15.46\end{array}$ & $\begin{array}{c}\$ \\
36.92 \\
\end{array}$ \\
\hline Colombia & $\$$ & 243.23 & $\$$ & 170.71 & $\begin{array}{l}\$ \\
95.34\end{array}$ & $\begin{array}{c}\$ \\
212.16\end{array}$ \\
\hline Comoros & $\$$ & 47.50 & $\$$ & 35.66 & $\begin{array}{l}\$ \\
19.64\end{array}$ & $\begin{array}{c}\$ \\
42.39 \\
\end{array}$ \\
\hline Congo, Dem. Rep. & $\$$ & 41.27 & $\$$ & 30.99 & $\$$ & $\$$ \\
\hline
\end{tabular}


medRxiv preprint doi: https://doi.org/10.1101/2020.08.23.20180299; this version posted August 25, 2020. The copyright holder for this preprint (which was not certified by peer review) is the author/funder, who has granted medRxiv a license to display the preprint in perpetuity.

It is made available under a CC-BY-NC-ND 4.0 International license.

\begin{tabular}{|c|c|c|c|c|c|c|}
\hline & & & & & 15.05 & 36.49 \\
\hline Congo, Rep. & $\$$ & 52.39 & $\$$ & 40.41 & $\begin{array}{l}\$ \\
24.93\end{array}$ & $\begin{array}{l}\$ \\
47.65\end{array}$ \\
\hline Costa Rica & $\$$ & 300.45 & $\$$ & 208.46 & $\begin{array}{l}\$ \\
121.44\end{array}$ & $\begin{array}{l}\$ \\
261.18\end{array}$ \\
\hline Cote d'Ivoire & $\$$ & 47.48 & $\$$ & 35.51 & $\begin{array}{l}\$ \\
16.05\end{array}$ & $\begin{array}{l} \\
42.28\end{array}$ \\
\hline Cuba & $\$$ & 177.94 & $\$$ & 124.79 & $\begin{array}{l}\$ \\
74.54\end{array}$ & $\begin{array}{l}\$ \\
155.92\end{array}$ \\
\hline Djibouti & $\$$ & 63.38 & $\$$ & 48.87 & $\begin{array}{c}\$ \\
27.93\end{array}$ & $\begin{array}{l}\$ \\
57.73\end{array}$ \\
\hline Dominican Republic & $\$$ & 241.43 & $\$$ & 172.09 & $\begin{array}{l}\$ \\
100.59\end{array}$ & $\begin{array}{l}\$ \\
212.38\end{array}$ \\
\hline Ecuador & $\$$ & 192.89 & $\$$ & 139.37 & $\begin{array}{c}\$ \\
80.49\end{array}$ & $\begin{array}{l}\$ \\
170.24\end{array}$ \\
\hline Egypt, Arab Rep. & $\$$ & 65.48 & $\$$ & 46.51 & $\begin{array}{l}\$ \\
24.28\end{array}$ & $\begin{array}{l}\$ \\
57.61\end{array}$ \\
\hline El Salvador & $\$$ & 92.14 & $\$$ & 67.64 & $\begin{array}{l}\$ \\
38.64\end{array}$ & $\begin{array}{l}\$ \\
82.62\end{array}$ \\
\hline Equatorial Guinea & $\$$ & 176.53 & $\$$ & 136.72 & $\begin{array}{l}\$ \\
76.69\end{array}$ & $\begin{array}{l}\$ \\
160.93\end{array}$ \\
\hline Eswatini & $\$$ & 65.63 & $\$$ & 49.31 & $\begin{array}{c}\$ \\
23.26\end{array}$ & $\begin{array}{c}\$ \\
59.24 \\
\end{array}$ \\
\hline Ethiopia & $\$$ & 69.49 & $\$$ & 51.53 & $\begin{array}{l}\$ \\
28.21\end{array}$ & $\begin{array}{l}\$ \\
61.19\end{array}$ \\
\hline Gabon & $\$$ & 168.26 & $\$$ & 126.68 & $\begin{array}{l}\$ \\
80.05\end{array}$ & $\begin{array}{l}\$ \\
151.45\end{array}$ \\
\hline Gambia, The & $\$$ & 52.29 & $\$$ & 39.81 & $\begin{array}{l}\$ \\
22.82\end{array}$ & $\begin{array}{l}\$ \\
46.54\end{array}$ \\
\hline Ghana & $\$$ & 54.47 & $\$$ & 41.05 & $\begin{array}{l}\$ \\
18.71\end{array}$ & $\begin{array}{l}\$ \\
48.84\end{array}$ \\
\hline Guatemala & $\$$ & 118.41 & $\$$ & 86.00 & $\begin{array}{l}\$ \\
49.53\end{array}$ & $\begin{array}{l}\$ \\
104.09\end{array}$ \\
\hline Guinea & $\$$ & 54.11 & $\$$ & 41.04 & $\begin{array}{l}\$ \\
22.89\end{array}$ & $\begin{array}{l}\$ \\
48.41\end{array}$ \\
\hline Guinea-Bissau & $\$$ & 48.97 & $\$$ & 37.35 & $\begin{array}{l}\$ \\
22.37\end{array}$ & $\begin{array}{l}\$ \\
43.93\end{array}$ \\
\hline Haiti & $\$$ & 58.37 & $\$$ & 43.34 & $\begin{array}{l}\$ \\
25.19\end{array}$ & $\begin{array}{l} \\
51.95\end{array}$ \\
\hline Honduras & $\$$ & 61.56 & $\$$ & 46.89 & $\begin{array}{c}\$ \\
28.43\end{array}$ & $\begin{array}{c}\$ \\
55.70\end{array}$ \\
\hline India & $\$$ & 93.33 & $\$$ & 70.12 & $\begin{array}{l}\$ \\
42.75 \\
\end{array}$ & $\begin{array}{l}\$ \\
84.06\end{array}$ \\
\hline Iran, Islamic Rep. & $\$$ & 89.86 & $\$$ & 60.68 & $\begin{array}{l}\$ \\
34.65\end{array}$ & $\begin{array}{c} \\
76.81\end{array}$ \\
\hline 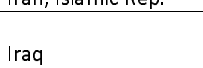 & $\$$ & 151.13 & $\$$ & 112.54 & $\begin{array}{l}\$ \$ \\
62.28\end{array}$ & $\begin{array}{l}\$ \\
134.73\end{array}$ \\
\hline Jordan & $\$$ & 104.75 & $\$$ & 75.06 & $\begin{array}{l}\$ \\
46.21\end{array}$ & $\begin{array}{l}\$ \\
91.30\end{array}$ \\
\hline Kenya & $\$$ & 80.49 & $\$$ & 65.63 & $\begin{array}{c}\$ \\
42.54 \\
\end{array}$ & $\begin{array}{c}\$ \\
74.51\end{array}$ \\
\hline Lebanon & $\$$ & 233.83 & $\$$ & 170.83 & $\begin{array}{l}\$ \\
115.21\end{array}$ & $\begin{array}{l}\$ \\
207.94\end{array}$ \\
\hline Lesotho & $\$$ & 48.88 & $\$$ & 35.07 & $\begin{array}{l}\$ \\
16.17\end{array}$ & $\begin{array}{l}\$ \\
42.60\end{array}$ \\
\hline Liberia & $\$$ & 47.76 & $\$$ & 36.12 & $\begin{array}{c}\$ \\
21.52\end{array}$ & $\begin{array}{l}\$ \\
42.59\end{array}$ \\
\hline Libya & $\$$ & 197.28 & $\$$ & 137.16 & $\begin{array}{l}\$ \\
72.53\end{array}$ & $\begin{array}{l}\$ \\
170.74\end{array}$ \\
\hline Madagascar & $\$$ & 47.93 & $\$$ & 35.39 & $\begin{array}{l}\$ \\
18.97\end{array}$ & $\begin{array}{l}\$ \\
42.20\end{array}$ \\
\hline Malawi & $\$$ & 40.95 & $\$$ & 30.27 & $\begin{array}{l}\$ \\
16.15\end{array}$ & $\begin{array}{l}\$ \$ \\
35.92\end{array}$ \\
\hline Mali & $\$$ & 45.49 & $\$$ & 32.63 & $\begin{array}{l}\$ \\
16.59\end{array}$ & $\begin{array}{l}\$ \\
39.60\end{array}$ \\
\hline Mauritania & $\$$ & 46.96 & $\$$ & 35.86 & $\begin{array}{l}\$ \\
22.55\end{array}$ & $\begin{array}{l}\$ \\
42.28\end{array}$ \\
\hline Mauritius & $\$$ & 424.52 & $\$$ & 293.12 & $\begin{array}{l}\$ \\
185.65\end{array}$ & $\begin{array}{l}\$ \\
367.56\end{array}$ \\
\hline Morocco & $\$$ & 90.69 & $\$$ & 67.48 & $\begin{array}{l}\$ \\
\$ 2.16\end{array}$ & $\begin{array}{l}\$ \\
81.61\end{array}$ \\
\hline Mozambique & $\$$ & 43.10 & $\$$ & 31.90 & $\begin{array}{l}\$ \\
17.03\end{array}$ & $\begin{array}{l}\$ \\
37.95\end{array}$ \\
\hline Namibia & $\$$ & 107.73 & $\$$ & 79.92 & $\begin{array}{l}\$ \\
37.34\end{array}$ & $\begin{array}{c}\$ \\
95.34\end{array}$ \\
\hline Nepal & $\$$ & 73.35 & $\$$ & 54.18 & $\begin{array}{l}\$ \\
27.55\end{array}$ & $\begin{array}{l}\$ \\
65.05\end{array}$ \\
\hline Nicaragua & $\$$ & 59.49 & $\$$ & 44.13 & $\begin{array}{l}\$ \\
24.50\end{array}$ & $\begin{array}{l}\$ \\
52.93\end{array}$ \\
\hline
\end{tabular}


medRxiv preprint doi: https://doi.org/10.1101/2020.08.23.20180299; this version posted August 25, 2020. The copyright holder for this preprint (which was not certified by peer review) is the author/funder, who has granted medRxiv a license to display the preprint in perpetuity.

It is made available under a CC-BY-NC-ND 4.0 International license .

\begin{tabular}{|c|c|c|c|c|c|c|}
\hline Niger & $\$$ & 37.52 & $\$$ & 26.71 & $\begin{array}{l}\$ \\
13.53 \\
\end{array}$ & $\begin{array}{c}\$ \\
32.35 \\
\end{array}$ \\
\hline Nigeria & $\$$ & 52.31 & $\$$ & 40.65 & $\begin{array}{l}\$ \\
22.70\end{array}$ & $\begin{array}{l}\$ \\
47.61 \\
\end{array}$ \\
\hline Pakistan & $\$$ & 44.49 & $\$$ & 32.37 & $\begin{array}{l}\$ \\
18.38\end{array}$ & $\begin{array}{l}\$ \\
38.84\end{array}$ \\
\hline Paraguay & $\$$ & 173.69 & $\$$ & 122.32 & $\begin{array}{c}\$ \\
63.04\end{array}$ & $\begin{array}{l}\$ \\
151.38\end{array}$ \\
\hline Peru & $\$$ & 223.50 & $\$$ & 155.85 & $\begin{array}{c}\$ \\
79.73\end{array}$ & $\begin{array}{c}\$ \\
193.43\end{array}$ \\
\hline Rwanda & $\$$ & 51.04 & $\$$ & 38.32 & $\begin{array}{l}\$ \\
21.12\end{array}$ & $\begin{array}{l}\$ \\
45.36\end{array}$ \\
\hline Sao Tome and Principe & $\$$ & 52.24 & $\$$ & 41.08 & $\begin{array}{l}\$ \\
23.90\end{array}$ & $\begin{array}{l}\$ \$ \\
47.59\end{array}$ \\
\hline Senegal & $\$$ & 48.94 & $\$$ & 37.55 & $\begin{array}{l}\$ \\
21.67\end{array}$ & $\begin{array}{l}\$ \\
44.10\end{array}$ \\
\hline Sierra Leone & $\$$ & 46.26 & $\$$ & 35.06 & $\begin{array}{c}\$ \\
20.91\end{array}$ & $\begin{array}{c}\$ \\
41.31\end{array}$ \\
\hline Somalia & $\$$ & 34.07 & $\$$ & 25.41 & $\begin{array}{l}\$ \\
13.44\end{array}$ & $\begin{array}{c}\$ \\
29.90\end{array}$ \\
\hline South Africa & $\$$ & 192.41 & $\$$ & 144.25 & $\begin{array}{c}\$ \\
85.86\end{array}$ & $\begin{array}{l}\$ \\
174.88\end{array}$ \\
\hline South Sudan & $\$$ & 41.66 & $\$$ & 31.55 & $\begin{array}{l}\$ \\
15.04\end{array}$ & $\begin{array}{l}\$ \\
37.25\end{array}$ \\
\hline Sri Lanka & $\$$ & 262.03 & $\$$ & 187.59 & $\begin{array}{l}\$ \\
121.06\end{array}$ & $\begin{array}{c}\$ \\
230.21\end{array}$ \\
\hline Sudan & $\$$ & 46.53 & $\$$ & 35.05 & $\begin{array}{l}\$ \\
19.11\end{array}$ & $\begin{array}{l}\$ \\
41.44\end{array}$ \\
\hline Syrian Arab Republic & $\$$ & 51.82 & $\$$ & 40.43 & $\begin{array}{l}\$ \\
24.75\end{array}$ & $\begin{array}{l}\$ \\
47.54 \\
\end{array}$ \\
\hline Tajikistan & $\$$ & 56.95 & $\$$ & 40.38 & $\begin{array}{l}\$ \\
20.72\end{array}$ & $\begin{array}{l}\$ \\
49.42\end{array}$ \\
\hline Tanzania & $\$$ & 57.34 & $\$$ & 43.62 & $\begin{array}{l}\$ \\
24.19\end{array}$ & $\begin{array}{c}4.42 \\
\$ \\
51.55\end{array}$ \\
\hline Togo & $\$$ & 47.51 & $\$$ & 36.17 & $\begin{array}{c}\$ \\
19.77\end{array}$ & $\begin{array}{l}\$ \\
42.43\end{array}$ \\
\hline Tunisia & $\$$ & 96.55 & $\$$ & 68.87 & $\begin{array}{l}\$ \\
40.42\end{array}$ & $\begin{array}{c}\$ \\
85.31\end{array}$ \\
\hline Turkey & $\$$ & 396.71 & $\$$ & 287.41 & $\begin{array}{l}\$ \\
189.07\end{array}$ & $\begin{array}{l}\$ \\
351.45\end{array}$ \\
\hline Uganda & $\$$ & 47.00 & $\$$ & 37.19 & $\begin{array}{l}\$ \\
32.95\end{array}$ & $\begin{array}{l}\$ \\
43.30\end{array}$ \\
\hline Venezuela, RB & $\$$ & 151.61 & $\$$ & 110.73 & $\begin{array}{l}\$ \\
70.13\end{array}$ & $\begin{array}{l}\$ \\
136.26\end{array}$ \\
\hline West Bank and Gaza & $\$$ & 56.41 & $\$$ & 42.82 & $\begin{array}{l}\$ \\
26.25\end{array}$ & $\begin{array}{c}\$ \\
51.07\end{array}$ \\
\hline Yemen, Rep. & $\$$ & 46.60 & $\$$ & 32.94 & $\begin{array}{l}\$ \$ \\
16.37\end{array}$ & $\begin{array}{l}\$ \\
40.04\end{array}$ \\
\hline Zambia & $\$$ & 43.13 & $\$$ & 33.22 & $\begin{array}{l}\$ \\
16.20\end{array}$ & $\begin{array}{c}\$ \\
38.91\end{array}$ \\
\hline Zimbabwe & $\$$ & 52.44 & $\$$ & 42.80 & $\begin{array}{l}\$ \\
40.49\end{array}$ & $\begin{array}{c}\$ \\
49.55\end{array}$ \\
\hline
\end{tabular}

Table SR3. Total annual costs per country (2019 US\$)

\begin{tabular}{|c|c|c|c|c|}
\hline \multirow{2}{*}{ Country } & \multicolumn{4}{|c|}{ Total Annual Costs per Country (2019 US\$) } \\
\hline & Scenario 1 & Scenario 4 & Scenario 14 & Scenario 49 \\
\hline Afghanistan & $1,595,006,688$ & $1,177,904,724$ & $664,951,614$ & $1,399,789,895$ \\
\hline Algeria & $7,198,927,335$ & $4,797,609,340$ & $2,505,281,318$ & $6,084,882,367$ \\
\hline Angola & $1,703,804,507$ & $1,335,741,419$ & $651,587,956$ & $1,567,094,020$ \\
\hline Argentina & $16,990,791,939$ & $12,132,966,200$ & $7,694,951,524$ & $15,001,801,214$ \\
\hline Bangladesh & $9,758,429,693$ & $7,346,626,409$ & $4,162,921,283$ & $8,762,131,868$ \\
\hline Benin & $609,231,361$ & $463,323,321$ & $252,112,394$ & $544,803,973$ \\
\hline Bolivia & $828,941,402$ & $607,984,156$ & $272,020,642$ & $738,627,623$ \\
\hline
\end{tabular}


medRxiv preprint doi: https://doi.org/10.1101/2020.08.23.20180299; this version posted August 25, 2020. The copyright holder for this preprint (which was not certified by peer review) is the author/funder, who has granted medRxiv a license to display the preprint in perpetuity.

It is made available under a CC-BY-NC-ND 4.0 International license.

\begin{tabular}{|c|c|c|c|c|}
\hline Botswana & $421,205,098$ & $303,116,161$ & $171,230,132$ & $369,522,644$ \\
\hline Brazil & $57,156,312,626$ & $40,013,304,222$ & $22,894,275,352$ & $49,832,168,384$ \\
\hline Burkina Faso & $939,419,926$ & $719,748,836$ & $417,278,324$ & $840,119,232$ \\
\hline Burundi & $408,399,996$ & $304,544,873$ & $167,162,430$ & $359,734,439$ \\
\hline Cabo Verde & $39,247,227$ & $28,162,920$ & $15,780,012$ & $34,414,567$ \\
\hline Cambodia & $802,267,483$ & $566,274,013$ & $229,000,547$ & $690,985,182$ \\
\hline Cameroon & $1,154,599,786$ & $884,467,637$ & $484,883,954$ & $1,043,034,826$ \\
\hline Central African Republic & $182,433,804$ & $137,035,951$ & $83,939,501$ & $161,434,468$ \\
\hline Chad & $658,884,973$ & $471,303,516$ & $239,244,463$ & $571,410,658$ \\
\hline Colombia & $12,075,940,244$ & $8,475,322,329$ & $4,733,454,628$ & $10,533,617,075$ \\
\hline Comoros & $39,533,969$ & $29,677,244$ & $16,343,351$ & $35,281,984$ \\
\hline Congo, Dem. Rep. & $3,469,615,019$ & $2,605,619,705$ & $1,265,377,537$ & $3,067,476,753$ \\
\hline Congo, Rep. & $274,760,274$ & $211,912,215$ & $130,725,594$ & $249,919,561$ \\
\hline Costa Rica & $1,502,087,635$ & $1,042,161,571$ & $607,146,919$ & $1,305,750,553$ \\
\hline Cote d'Ivoire & $1,190,399,892$ & $890,204,132$ & $402,280,265$ & $1,059,989,337$ \\
\hline Cuba & $2,017,494,968$ & $1,414,934,979$ & $845,099,624$ & $1,767,876,988$ \\
\hline Djibouti & $60,775,939$ & $46,862,116$ & $26,784,302$ & $55,355,583$ \\
\hline Dominican Republic & $2,565,684,173$ & $1,828,777,716$ & $1,068,959,610$ & $2,256,950,992$ \\
\hline Ecuador & $3,295,395,303$ & $2,380,985,925$ & $1,375,043,118$ & $2,908,422,164$ \\
\hline Egypt, Arab Rep. & $6,445,190,291$ & $4,577,530,923$ & $2,389,669,661$ & $5,670,659,187$ \\
\hline EI Salvador & $591,579,720$ & $434,268,407$ & $248,098,581$ & $530,457,154$ \\
\hline Equatorial Guinea & $231,075,219$ & $178,959,540$ & $100,391,379$ & $210,653,340$ \\
\hline Eswatini & $74,567,166$ & $56,029,505$ & $26,432,806$ & $67,309,663$ \\
\hline Ethiopia & $7,589,595,244$ & $5,627,795,574$ & $3,081,548,357$ & $6,683,485,989$ \\
\hline Gabon & $356,596,194$ & $268,464,549$ & $169,638,184$ & $320,961,919$ \\
\hline Gambia, The & $119,218,198$ & $90,763,358$ & $52,035,644$ & $106,107,646$ \\
\hline Ghana & $1,621,297,684$ & $1,221,891,384$ & $556,920,614$ & $1,453,846,283$ \\
\hline Guatemala & $2,042,320,674$ & $1,483,251,542$ & $854,210,602$ & $1,795,402,698$ \\
\hline Guinea & $671,793,449$ & $509,527,263$ & $284,208,408$ & $600,953,264$ \\
\hline Guinea-Bissau & $91,777,597$ & $70,013,569$ & $41,923,342$ & $82,337,549$ \\
\hline Haiti & $649,227,561$ & $482,130,225$ & $280,151,525$ & $577,852,090$ \\
\hline Honduras & $590,251,293$ & $449,603,344$ & $272,545,259$ & $534,058,049$ \\
\hline India & $126,241,607,727$ & $94,845,714,832$ & $57,820,205,064$ & $113,697,199,394$ \\
\hline Iran, Islamic Rep. & $7,350,914,852$ & $4,963,443,606$ & $2,834,585,552$ & $6,283,486,292$ \\
\hline Iraq & $5,808,461,053$ & $4,325,177,524$ & $2,393,641,505$ & $5,178,286,305$ \\
\hline Jordan & $1,042,846,812$ & $747,309,105$ & $460,019,255$ & $908,958,207$ \\
\hline Kenya & $4,136,465,948$ & $3,372,960,785$ & $2,186,416,908$ & $3,829,322,849$ \\
\hline Lebanon & $1,601,468,785$ & $1,169,981,518$ & $789,069,252$ & $1,424,189,709$ \\
\hline Lesotho & $103,041,897$ & $73,935,288$ & $34,089,165$ & $89,815,951$ \\
\hline Liberia & $230,138,172$ & $174,077,339$ & $103,699,634$ & $205,216,814$ \\
\hline Libya & $1,317,562,603$ & $916,036,535$ & $484,376,095$ & $1,140,287,493$ \\
\hline
\end{tabular}


medRxiv preprint doi: https://doi.org/10.1101/2020.08.23.20180299; this version posted August 25, 2020. The copyright holder for this preprint (which was not certified by peer review) is the author/funder, who has granted medRxiv a license to display the preprint in perpetuity.

It is made available under a CC-BY-NC-ND 4.0 International license .

\begin{tabular}{|c|c|c|c|c|}
\hline Madagascar & $1,258,737,705$ & $929,396,816$ & $498,242,703$ & $1,108,391,195$ \\
\hline Malawi & $742,886,055$ & $549,184,096$ & $293,026,156$ & $651,787,586$ \\
\hline Mali & $867,812,732$ & $622,492,840$ & $316,411,256$ & $755,421,554$ \\
\hline Mauritania & $206,771,925$ & $157,899,063$ & $99,305,744$ & $186,164,959$ \\
\hline Mauritius & $537,143,681$ & $370,884,267$ & $234,900,728$ & $465,076,712$ \\
\hline Mexico & $34,115,201,144$ & $24,625,827,163$ & $15,782,810,293$ & $30,216,622,750$ \\
\hline Morocco & $3,267,515,013$ & $2,431,152,726$ & $1,519,075,135$ & $2,940,380,785$ \\
\hline Mozambique & $1,271,233,930$ & $940,866,088$ & $502,228,251$ & $1,119,434,522$ \\
\hline Namibia & $263,758,483$ & $195,667,209$ & $91,410,118$ & $233,404,625$ \\
\hline Nepal & $2,060,381,058$ & $1,521,796,884$ & $773,785,915$ & $1,827,252,564$ \\
\hline Nicaragua & $384,638,289$ & $285,335,078$ & $158,382,871$ & $342,217,217$ \\
\hline Niger & $842,039,860$ & $599,341,108$ & $303,663,836$ & $725,945,647$ \\
\hline Nigeria & $10,247,169,054$ & $7,962,255,548$ & $4,445,505,322$ & $9,326,514,000$ \\
\hline Pakistan & $9,441,146,849$ & $6,868,761,344$ & $3,901,350,552$ & $8,242,469,426$ \\
\hline Paraguay & $1,208,234,685$ & $850,884,034$ & $438,489,428$ & $1,053,013,278$ \\
\hline Peru & $7,149,625,157$ & $4,985,660,537$ & $2,550,356,751$ & $6,187,688,798$ \\
\hline Rwanda & $627,950,026$ & $471,398,249$ & $259,768,907$ & $558,014,561$ \\
\hline Sao Tome and Principe & $11,024,075$ & $8,669,252$ & $5,044,582$ & $10,042,513$ \\
\hline Senegal & $775,913,554$ & $595,256,052$ & $343,558,437$ & $699,179,799$ \\
\hline Sierra Leone & $353,894,747$ & $268,216,325$ & $159,990,783$ & $316,044,465$ \\
\hline Somalia & $511,309,434$ & $381,354,484$ & $201,668,843$ & $448,722,521$ \\
\hline South Africa & $11,117,131,659$ & $8,334,446,288$ & $4,960,920,248$ & $10,104,268,308$ \\
\hline South Sudan & $457,247,980$ & $346,307,159$ & $165,112,181$ & $408,857,053$ \\
\hline Sri Lanka & $5,678,100,834$ & $4,065,040,082$ & $2,623,300,200$ & $4,988,596,480$ \\
\hline Sudan & $1,944,960,100$ & $1,465,299,051$ & $798,971,465$ & $1,732,370,209$ \\
\hline Syrian Arab Republic & $876,059,081$ & $683,467,982$ & $418,397,444$ & $803,740,032$ \\
\hline Tajikistan & $518,284,390$ & $367,451,171$ & $188,545,177$ & $449,773,206$ \\
\hline Tanzania & $3,229,078,738$ & $2,456,724,397$ & $1,362,187,767$ & $2,903,091,232$ \\
\hline Togo & $374,774,447$ & $285,340,906$ & $155,932,903$ & $334,768,272$ \\
\hline Tunisia & $1,116,665,762$ & $796,503,718$ & $467,489,081$ & $986,577,100$ \\
\hline Turkey & $32,657,064,433$ & $23,659,504,785$ & $15,564,550,540$ & $28,931,632,252$ \\
\hline Uganda & $2,007,915,732$ & $1,588,879,797$ & $1,407,932,788$ & $1,849,941,504$ \\
\hline Venezuela, RB & $4,377,122,572$ & $3,196,922,891$ & $2,024,556,702$ & $3,933,821,378$ \\
\hline West Bank and Gaza & $257,722,952$ & $195,636,416$ & $119,932,408$ & $233,328,972$ \\
\hline Yemen, Rep. & $1,328,176,781$ & $938,682,001$ & $466,480,766$ & $1,141,026,798$ \\
\hline Zambia & $748,338,033$ & $576,387,647$ & $281,151,582$ & $675,092,378$ \\
\hline Zimbabwe & $757,131,633$ & $617,966,425$ & $584,680,182$ & $715,488,782$ \\
\hline
\end{tabular}


medRxiv preprint doi: https://doi.org/10.1101/2020.08.23.20180299; this version posted August 25, 2020. The copyright holder for this preprint (which was not certified by peer review) is the author/funder, who has granted medRxiv a license to display the preprint in perpetuity.

It is made available under a CC-BY-NC-ND 4.0 International license .

Table SR4: Health System Costs of COVID 19 Response per capita as \% of GDP per capita (nominal)

\begin{tabular}{|c|c|c|c|c|}
\hline Country & Scenario 1 & Scenario 4 & Scenario 14 & Scenario 49 \\
\hline Afghanistan & $8.24 \%$ & $6.08 \%$ & $3.43 \%$ & $7.23 \%$ \\
\hline Algeria & $4.14 \%$ & $2.76 \%$ & $1.44 \%$ & $3.50 \%$ \\
\hline Angola & $1.61 \%$ & $1.26 \%$ & $0.62 \%$ & $1.48 \%$ \\
\hline Argentina & $3.27 \%$ & $2.33 \%$ & $1.48 \%$ & $2.89 \%$ \\
\hline Bangladesh & $3.56 \%$ & $2.68 \%$ & $1.52 \%$ & $3.20 \%$ \\
\hline Benin & $5.88 \%$ & $4.47 \%$ & $2.43 \%$ & $5.26 \%$ \\
\hline Bolivia & $2.06 \%$ & $1.51 \%$ & $0.68 \%$ & $1.83 \%$ \\
\hline Botswana & $2.26 \%$ & $1.63 \%$ & $0.92 \%$ & $1.98 \%$ \\
\hline Brazil & $3.06 \%$ & $2.14 \%$ & $1.23 \%$ & $2.67 \%$ \\
\hline Burkina Faso & $6.65 \%$ & $5.10 \%$ & $2.95 \%$ & $5.95 \%$ \\
\hline Burundi & $13.45 \%$ & $10.03 \%$ & $5.50 \%$ & $11.85 \%$ \\
\hline Cabo Verde & $1.99 \%$ & $1.42 \%$ & $0.80 \%$ & $1.74 \%$ \\
\hline Cambodia & $3.27 \%$ & $2.31 \%$ & $0.93 \%$ & $2.82 \%$ \\
\hline Cameroon & $2.99 \%$ & $2.29 \%$ & $1.25 \%$ & $2.70 \%$ \\
\hline Central African Republic & $8.22 \%$ & $6.17 \%$ & $3.78 \%$ & $7.27 \%$ \\
\hline Chad & $5.84 \%$ & $4.18 \%$ & $2.12 \%$ & $5.07 \%$ \\
\hline Colombia & $3.65 \%$ & $2.56 \%$ & $1.43 \%$ & $3.18 \%$ \\
\hline Comoros & $3.36 \%$ & $2.52 \%$ & $1.39 \%$ & $3.00 \%$ \\
\hline Congo, Dem. Rep. & $7.35 \%$ & $5.52 \%$ & $2.68 \%$ & $6.50 \%$ \\
\hline Congo, Rep. & $2.44 \%$ & $1.88 \%$ & $1.16 \%$ & $2.22 \%$ \\
\hline Costa Rica & $2.50 \%$ & $1.73 \%$ & $1.01 \%$ & $2.17 \%$ \\
\hline Cote d'Ivoire & $2.77 \%$ & $2.07 \%$ & $0.94 \%$ & $2.46 \%$ \\
\hline Cuba & $2.02 \%$ & $1.41 \%$ & $0.84 \%$ & $1.77 \%$ \\
\hline Djibouti & $2.06 \%$ & $1.59 \%$ & $0.91 \%$ & $1.87 \%$ \\
\hline Dominican Republic & $3.00 \%$ & $2.14 \%$ & $1.25 \%$ & $2.64 \%$ \\
\hline Ecuador & $3.04 \%$ & $2.20 \%$ & $1.27 \%$ & $2.68 \%$ \\
\hline Egypt, Arab Rep. & $2.57 \%$ & $1.82 \%$ & $0.95 \%$ & $2.26 \%$ \\
\hline El Salvador & $2.27 \%$ & $1.67 \%$ & $0.95 \%$ & $2.04 \%$ \\
\hline Equatorial Guinea & $1.72 \%$ & $1.33 \%$ & $0.75 \%$ & $1.57 \%$ \\
\hline Eswatini & $1.58 \%$ & $1.19 \%$ & $0.56 \%$ & $1.43 \%$ \\
\hline Ethiopia & $9.00 \%$ & $6.67 \%$ & $3.65 \%$ & $7.92 \%$ \\
\hline Gabon & $2.12 \%$ & $1.59 \%$ & $1.01 \%$ & $1.90 \%$ \\
\hline Gambia, The & $7.30 \%$ & $5.56 \%$ & $3.19 \%$ & $6.50 \%$ \\
\hline Ghana & $2.47 \%$ & $1.86 \%$ & $0.85 \%$ & $2.22 \%$ \\
\hline Guatemala & $2.60 \%$ & $1.89 \%$ & $1.09 \%$ & $2.29 \%$ \\
\hline Guinea & $6.16 \%$ & $4.67 \%$ & $2.61 \%$ & $5.51 \%$ \\
\hline Guinea-Bissau & $6.29 \%$ & $4.80 \%$ & $2.88 \%$ & $5.65 \%$ \\
\hline Haiti & $6.72 \%$ & $4.99 \%$ & $2.90 \%$ & $5.98 \%$ \\
\hline Honduras & $2.46 \%$ & $1.88 \%$ & $1.14 \%$ & $2.23 \%$ \\
\hline India & $4.64 \%$ & $3.49 \%$ & $2.13 \%$ & $4.18 \%$ \\
\hline Iran, Islamic Rep. & $1.60 \%$ & $1.08 \%$ & $0.62 \%$ & $1.36 \%$ \\
\hline Jordan & $2.47 \%$ & $1.77 \%$ & $1.09 \%$ & $2.15 \%$ \\
\hline Kenya & $4.71 \%$ & $3.84 \%$ & $2.49 \%$ & $4.36 \%$ \\
\hline Lebanon & $2.83 \%$ & $2.07 \%$ & $1.39 \%$ & $2.51 \%$ \\
\hline Lesotho & $3.76 \%$ & $2.70 \%$ & $1.24 \%$ & $3.28 \%$ \\
\hline Liberia & $7.05 \%$ & $5.33 \%$ & $3.18 \%$ & $6.29 \%$ \\
\hline Libya & $2.72 \%$ & $1.89 \%$ & $1.00 \%$ & $2.36 \%$ \\
\hline Madagascar & $9.09 \%$ & $6.71 \%$ & $3.60 \%$ & $8.00 \%$ \\
\hline Malawi & $10.52 \%$ & $7.77 \%$ & $4.15 \%$ & $9.23 \%$ \\
\hline
\end{tabular}


medRxiv preprint doi: https://doi.org/10.1101/2020.08.23.20180299; this version posted August 25, 2020. The copyright holder for this preprint (which was not certified by peer review) is the author/funder, who has granted medRxiv a license to display the preprint in perpetuity.

It is made available under a CC-BY-NC-ND 4.0 International license.

\begin{tabular}{|c|c|c|c|c|}
\hline Mali & $5.06 \%$ & $3.63 \%$ & $1.84 \%$ & $4.40 \%$ \\
\hline Mauritania & $3.95 \%$ & $3.02 \%$ & $1.90 \%$ & $3.56 \%$ \\
\hline Mauritius & $3.78 \%$ & $2.61 \%$ & $1.65 \%$ & $3.27 \%$ \\
\hline Mexico & $2.79 \%$ & $2.02 \%$ & $1.29 \%$ & $2.48 \%$ \\
\hline Morocco & $2.80 \%$ & $2.08 \%$ & $1.30 \%$ & $2.52 \%$ \\
\hline Mozambique & $8.64 \%$ & $6.39 \%$ & $3.41 \%$ & $7.61 \%$ \\
\hline Namibia & $1.82 \%$ & $1.35 \%$ & $0.63 \%$ & $1.61 \%$ \\
\hline Nepal & $7.09 \%$ & $5.24 \%$ & $2.66 \%$ & $6.29 \%$ \\
\hline Nicaragua & $2.93 \%$ & $2.18 \%$ & $1.21 \%$ & $2.61 \%$ \\
\hline Niger & $9.06 \%$ & $6.45 \%$ & $3.27 \%$ & $7.81 \%$ \\
\hline Nigeria & $2.58 \%$ & $2.00 \%$ & $1.12 \%$ & $2.35 \%$ \\
\hline Pakistan & $3.00 \%$ & $2.18 \%$ & $1.24 \%$ & $2.62 \%$ \\
\hline Paraguay & $2.98 \%$ & $2.10 \%$ & $1.08 \%$ & $2.60 \%$ \\
\hline Peru & $3.22 \%$ & $2.25 \%$ & $1.15 \%$ & $2.79 \%$ \\
\hline Rwanda & $6.60 \%$ & $4.96 \%$ & $2.73 \%$ & $5.87 \%$ \\
\hline Sao Tome and Principe & $2.61 \%$ & $2.05 \%$ & $1.19 \%$ & $2.38 \%$ \\
\hline Senegal & $3.22 \%$ & $2.47 \%$ & $1.42 \%$ & $2.90 \%$ \\
\hline Sierra Leone & $8.66 \%$ & $6.57 \%$ & $3.92 \%$ & $7.74 \%$ \\
\hline South Africa & $3.02 \%$ & $2.26 \%$ & $1.35 \%$ & $2.74 \%$ \\
\hline Sri Lanka & $6.39 \%$ & $4.57 \%$ & $2.95 \%$ & $5.61 \%$ \\
\hline Sudan & $4.76 \%$ & $3.59 \%$ & $1.96 \%$ & $4.24 \%$ \\
\hline Syrian Arab Republic & $2.55 \%$ & $1.99 \%$ & $1.22 \%$ & $2.34 \%$ \\
\hline Tajikistan & $6.89 \%$ & $4.88 \%$ & $2.51 \%$ & $5.98 \%$ \\
\hline Tanzania & $5.46 \%$ & $4.15 \%$ & $2.30 \%$ & $4.91 \%$ \\
\hline Togo & $6.99 \%$ & $5.32 \%$ & $2.91 \%$ & $6.25 \%$ \\
\hline Tunisia & $2.80 \%$ & $2.00 \%$ & $1.17 \%$ & $2.47 \%$ \\
\hline Turkey & $4.23 \%$ & $3.07 \%$ & $2.02 \%$ & $3.75 \%$ \\
\hline Venezuela, RB & $0.94 \%$ & $0.69 \%$ & $0.44 \%$ & $0.85 \%$ \\
\hline Yemen, Rep. & $4.93 \%$ & $3.49 \%$ & $1.73 \%$ & $4.24 \%$ \\
\hline Zambia & $2.80 \%$ & $2.16 \%$ & $1.05 \%$ & $2.53 \%$ \\
\hline Zimbabwe & $2.44 \%$ & $1.99 \%$ & $1.89 \%$ & $2.31 \%$ \\
\hline
\end{tabular}


medRxiv preprint doi: https://doi.org/10.1101/2020.08.23.20180299; this version posted August 25, 2020. The copyright holder for this preprint (which was not certified by peer review) is the author/funder, who has granted medRxiv a license to display the preprint in perpetuity.

It is made available under a CC-BY-NC-ND 4.0 International license.

Table SR5: Health System Costs of COVID 19 Response as \% of total health spending (excl. OOP)

\begin{tabular}{|c|c|c|c|c|}
\hline Country & Scenario 1 & Scenario 4 & Scenario 14 & Scenario 49 \\
\hline Afghanistan & $74.95 \%$ & $55.35 \%$ & $31.25 \%$ & $65.78 \%$ \\
\hline Algeria & $65.46 \%$ & $43.63 \%$ & $22.78 \%$ & $55.33 \%$ \\
\hline Angola & $58.08 \%$ & $45.53 \%$ & $22.21 \%$ & $53.42 \%$ \\
\hline Argentina & $39.98 \%$ & $28.55 \%$ & $18.11 \%$ & $35.30 \%$ \\
\hline Bangladesh & $176.74 \%$ & $133.06 \%$ & $75.40 \%$ & $158.70 \%$ \\
\hline Benin & $174.48 \%$ & $132.70 \%$ & $72.20 \%$ & $156.03 \%$ \\
\hline Bolivia & $34.28 \%$ & $25.14 \%$ & $11.25 \%$ & $30.54 \%$ \\
\hline Botswana & $49.18 \%$ & $35.39 \%$ & $19.99 \%$ & $43.15 \%$ \\
\hline Brazil & $26.86 \%$ & $18.80 \%$ & $10.76 \%$ & $23.42 \%$ \\
\hline Burkina Faso & $116.17 \%$ & $89.01 \%$ & $51.60 \%$ & $103.89 \%$ \\
\hline Burundi & $197.95 \%$ & $147.61 \%$ & $81.02 \%$ & $174.36 \%$ \\
\hline Cabo Verde & $45.37 \%$ & $32.56 \%$ & $18.24 \%$ & $39.79 \%$ \\
\hline Cambodia & $63.56 \%$ & $44.86 \%$ & $18.14 \%$ & $54.75 \%$ \\
\hline Cameroon & $71.03 \%$ & $54.41 \%$ & $29.83 \%$ & $64.16 \%$ \\
\hline Central African Republic & $238.96 \%$ & $179.50 \%$ & $109.95 \%$ & $211.46 \%$ \\
\hline Chad & $134.35 \%$ & $96.10 \%$ & $48.78 \%$ & $116.51 \%$ \\
\hline Colombia & $71.46 \%$ & $50.15 \%$ & $28.01 \%$ & $62.33 \%$ \\
\hline Comoros & $80.51 \%$ & $60.43 \%$ & $33.28 \%$ & $71.85 \%$ \\
\hline Congo, Dem. Rep. & $201.15 \%$ & $151.06 \%$ & $73.36 \%$ & $177.84 \%$ \\
\hline Congo, Rep. & $74.44 \%$ & $57.42 \%$ & $35.42 \%$ & $67.71 \%$ \\
\hline Costa Rica & $33.80 \%$ & $23.45 \%$ & $13.66 \%$ & $29.38 \%$ \\
\hline Cote d'Ivoire & $70.27 \%$ & $52.55 \%$ & $23.75 \%$ & $62.58 \%$ \\
\hline Cuba & $18.33 \%$ & $12.86 \%$ & $7.68 \%$ & $16.06 \%$ \\
\hline Djibouti & $90.30 \%$ & $69.62 \%$ & $39.79 \%$ & $82.24 \%$ \\
\hline Dominican Republic & $58.29 \%$ & $41.55 \%$ & $24.29 \%$ & $51.28 \%$ \\
\hline Ecuador & $38.21 \%$ & $27.61 \%$ & $15.94 \%$ & $33.73 \%$ \\
\hline Egypt, Arab Rep. & $49.99 \%$ & $35.51 \%$ & $18.54 \%$ & $43.99 \%$ \\
\hline El Salvador & $31.35 \%$ & $23.02 \%$ & $13.15 \%$ & $28.11 \%$ \\
\hline Equatorial Guinea & $62.74 \%$ & $48.59 \%$ & $27.26 \%$ & $57.19 \%$ \\
\hline Eswatini & $29.75 \%$ & $22.36 \%$ & $10.55 \%$ & $26.86 \%$ \\
\hline Ethiopia & $252.46 \%$ & $187.20 \%$ & $102.50 \%$ & $222.32 \%$ \\
\hline Gabon & $76.36 \%$ & $57.49 \%$ & $36.33 \%$ & $68.73 \%$ \\
\hline Gambia, The & $249.81 \%$ & $190.19 \%$ & $109.04 \%$ & $222.34 \%$ \\
\hline Ghana & $80.68 \%$ & $60.80 \%$ & $27.71 \%$ & $72.35 \%$ \\
\hline Guatemala & $49.06 \%$ & $35.63 \%$ & $20.52 \%$ & $43.13 \%$ \\
\hline Guinea & $144.46 \%$ & $109.57 \%$ & $61.12 \%$ & $129.23 \%$ \\
\hline Guinea-Bissau & $125.38 \%$ & $95.65 \%$ & $57.27 \%$ & $112.48 \%$ \\
\hline Haiti & $154.72 \%$ & $114.90 \%$ & $66.77 \%$ & $137.71 \%$ \\
\hline Honduras & $30.85 \%$ & $23.50 \%$ & $14.25 \%$ & $27.91 \%$ \\
\hline India & $148.82 \%$ & $111.81 \%$ & $68.16 \%$ & $134.03 \%$ \\
\hline Iran, Islamic Rep. & $21.63 \%$ & $14.61 \%$ & $8.34 \%$ & $18.49 \%$ \\
\hline Jordan & $46.86 \%$ & $33.58 \%$ & $20.67 \%$ & $40.84 \%$ \\
\hline Kenya & $121.56 \%$ & $99.13 \%$ & $64.25 \%$ & $112.54 \%$ \\
\hline Lebanon & $35.31 \%$ & $25.80 \%$ & $17.40 \%$ & $31.41 \%$ \\
\hline Lesotho & $57.16 \%$ & $41.01 \%$ & $18.91 \%$ & $49.82 \%$ \\
\hline Liberia & $69.91 \%$ & $52.88 \%$ & $31.50 \%$ & $62.34 \%$ \\
\hline Libya & $63.11 \%$ & $43.88 \%$ & $23.20 \%$ & $54.62 \%$ \\
\hline Madagascar & $198.72 \%$ & $146.73 \%$ & $78.66 \%$ & $174.98 \%$ \\
\hline Malawi & $138.39 \%$ & $102.30 \%$ & $54.59 \%$ & $121.42 \%$ \\
\hline
\end{tabular}


medRxiv preprint doi: https://doi.org/10.1101/2020.08.23.20180299; this version posted August 25, 2020. The copyright holder for this preprint (which was not certified by peer review) is the author/funder, who has granted medRxiv a license to display the preprint in perpetuity.

\section{It is made available under a CC-BY-NC-ND 4.0 International license .}

\begin{tabular}{|c|c|c|c|c|}
\hline Mali & $152.71 \%$ & $109.54 \%$ & $55.68 \%$ & $132.94 \%$ \\
\hline Mauritania & $100.40 \%$ & $76.67 \%$ & $48.22 \%$ & $90.39 \%$ \\
\hline Mauritius & $76.75 \%$ & $53.00 \%$ & $33.57 \%$ & $66.46 \%$ \\
\hline Mexico & $58.54 \%$ & $42.26 \%$ & $27.08 \%$ & $51.85 \%$ \\
\hline Morocco & $52.90 \%$ & $39.36 \%$ & $24.59 \%$ & $47.60 \%$ \\
\hline Mozambique & $224.38 \%$ & $166.07 \%$ & $88.65 \%$ & $197.59 \%$ \\
\hline Namibia & $26.75 \%$ & $19.84 \%$ & $9.27 \%$ & $23.67 \%$ \\
\hline Nepal & $161.39 \%$ & $119.20 \%$ & $60.61 \%$ & $143.13 \%$ \\
\hline Nicaragua & $31.62 \%$ & $23.45 \%$ & $13.02 \%$ & $28.13 \%$ \\
\hline Niger & $165.40 \%$ & $117.73 \%$ & $59.65 \%$ & $142.60 \%$ \\
\hline Nigeria & $65.94 \%$ & $51.23 \%$ & $28.61 \%$ & $60.01 \%$ \\
\hline Pakistan & $112.40 \%$ & $81.78 \%$ & $46.45 \%$ & $98.13 \%$ \\
\hline Paraguay & $53.08 \%$ & $37.38 \%$ & $19.26 \%$ & $46.26 \%$ \\
\hline Peru & $70.63 \%$ & $49.25 \%$ & $25.19 \%$ & $61.13 \%$ \\
\hline Rwanda & $106.17 \%$ & $79.70 \%$ & $43.92 \%$ & $94.35 \%$ \\
\hline Sao Tome and Principe & $49.69 \%$ & $39.07 \%$ & $22.74 \%$ & $45.26 \%$ \\
\hline Senegal & $93.02 \%$ & $71.37 \%$ & $41.19 \%$ & $83.83 \%$ \\
\hline Sierra Leone & $53.60 \%$ & $40.62 \%$ & $24.23 \%$ & $47.87 \%$ \\
\hline South Africa & $44.94 \%$ & $33.69 \%$ & $20.05 \%$ & $40.84 \%$ \\
\hline Sri Lanka & $171.15 \%$ & $122.53 \%$ & $79.07 \%$ & $150.37 \%$ \\
\hline Sudan & $30.61 \%$ & $23.06 \%$ & $12.57 \%$ & $27.26 \%$ \\
\hline Syrian Arab Republic & $78.34 \%$ & $61.12 \%$ & $37.42 \%$ & $71.88 \%$ \\
\hline Tajikistan & $102.25 \%$ & $72.49 \%$ & $37.20 \%$ & $88.73 \%$ \\
\hline Tanzania & $161.51 \%$ & $122.88 \%$ & $68.13 \%$ & $145.21 \%$ \\
\hline Togo & $122.53 \%$ & $93.29 \%$ & $50.98 \%$ & $109.45 \%$ \\
\hline Tunisia & $37.64 \%$ & $26.85 \%$ & $15.76 \%$ & $33.26 \%$ \\
\hline Turkey & $84.65 \%$ & $61.33 \%$ & $40.34 \%$ & $74.99 \%$ \\
\hline Venezuela, RB & $9.61 \%$ & $7.02 \%$ & $4.44 \%$ & $8.63 \%$ \\
\hline Yemen, Rep. & $64.69 \%$ & $45.72 \%$ & $22.72 \%$ & $55.58 \%$ \\
\hline Zambia & $76.27 \%$ & $58.75 \%$ & $28.66 \%$ & $68.81 \%$ \\
\hline Zimbabwe & $55.82 \%$ & $45.56 \%$ & $43.10 \%$ & $52.75 \%$ \\
\hline
\end{tabular}


medRxiv preprint doi: https://doi.org/10.1101/2020.08.23.20180299; this version posted August 25, 2020. The copyright holder for this preprint (which was not certified by peer review) is the author/funder, who has granted medRxiv a license to display the preprint in perpetuity.

It is made available under a CC-BY-NC-ND 4.0 International license.

Table SR6: Health System Costs of COVID 19 Response as \% of total health spending (incl. OOP)

\begin{tabular}{|c|c|c|c|c|}
\hline Country & Scenario 1 & Scenario 4 & Scenario 14 & Scenario 49 \\
\hline Afghanistan & $42.25 \%$ & $31.20 \%$ & $17.61 \%$ & $37.08 \%$ \\
\hline Algeria & $50.02 \%$ & $33.33 \%$ & $17.41 \%$ & $42.28 \%$ \\
\hline Angola & $42.95 \%$ & $33.67 \%$ & $16.43 \%$ & $39.51 \%$ \\
\hline Argentina & $34.52 \%$ & $24.65 \%$ & $15.64 \%$ & $30.48 \%$ \\
\hline Bangladesh & $102.82 \%$ & $77.41 \%$ & $43.86 \%$ & $92.33 \%$ \\
\hline Benin & $121.61 \%$ & $92.48 \%$ & $50.32 \%$ & $108.75 \%$ \\
\hline Bolivia & $26.77 \%$ & $19.64 \%$ & $8.79 \%$ & $23.86 \%$ \\
\hline Botswana & $46.73 \%$ & $33.63 \%$ & $19.00 \%$ & $41.00 \%$ \\
\hline Brazil & $18.71 \%$ & $13.10 \%$ & $7.49 \%$ & $16.31 \%$ \\
\hline Burkina Faso & $88.42 \%$ & $67.74 \%$ & $39.27 \%$ & $79.07 \%$ \\
\hline Burundi & $151.67 \%$ & $113.10 \%$ & $62.08 \%$ & $133.59 \%$ \\
\hline Cabo Verde & $36.01 \%$ & $25.84 \%$ & $14.48 \%$ & $31.57 \%$ \\
\hline Cambodia & $40.09 \%$ & $28.30 \%$ & $11.44 \%$ & $34.53 \%$ \\
\hline Cameroon & $41.90 \%$ & $32.10 \%$ & $17.60 \%$ & $37.85 \%$ \\
\hline Central African Republic & $167.01 \%$ & $125.45 \%$ & $76.84 \%$ & $147.79 \%$ \\
\hline Chad & $83.37 \%$ & $59.63 \%$ & $30.27 \%$ & $72.30 \%$ \\
\hline Colombia & $59.47 \%$ & $41.74 \%$ & $23.31 \%$ & $51.88 \%$ \\
\hline Comoros & $46.50 \%$ & $34.91 \%$ & $19.22 \%$ & $41.50 \%$ \\
\hline Congo, Dem. Rep. & $146.36 \%$ & $109.92 \%$ & $53.38 \%$ & $129.40 \%$ \\
\hline Congo, Rep. & $49.72 \%$ & $38.35 \%$ & $23.66 \%$ & $45.23 \%$ \\
\hline Costa Rica & $27.67 \%$ & $19.20 \%$ & $11.19 \%$ & $24.06 \%$ \\
\hline Cote d'Ivoire & $50.14 \%$ & $37.50 \%$ & $16.95 \%$ & $44.65 \%$ \\
\hline Cuba & $16.62 \%$ & $11.66 \%$ & $6.96 \%$ & $14.56 \%$ \\
\hline Djibouti & $71.80 \%$ & $55.36 \%$ & $31.64 \%$ & $65.39 \%$ \\
\hline Dominican Republic & $40.31 \%$ & $28.73 \%$ & $16.79 \%$ & $35.46 \%$ \\
\hline Ecuador & $27.20 \%$ & $19.65 \%$ & $11.35 \%$ & $24.01 \%$ \\
\hline Egypt, Arab Rep. & $30.86 \%$ & $21.92 \%$ & $11.44 \%$ & $27.15 \%$ \\
\hline EI Salvador & $24.66 \%$ & $18.10 \%$ & $10.34 \%$ & $22.11 \%$ \\
\hline Equatorial Guinea & $36.30 \%$ & $28.11 \%$ & $15.77 \%$ & $33.09 \%$ \\
\hline Eswatini & $27.07 \%$ & $20.34 \%$ & $9.60 \%$ & $24.44 \%$ \\
\hline Ethiopia & $183.71 \%$ & $136.22 \%$ & $74.59 \%$ & $161.78 \%$ \\
\hline Gabon & $62.33 \%$ & $46.92 \%$ & $29.65 \%$ & $56.10 \%$ \\
\hline Gambia, The & $202.13 \%$ & $153.88 \%$ & $88.22 \%$ & $179.90 \%$ \\
\hline Ghana & $58.54 \%$ & $44.12 \%$ & $20.11 \%$ & $52.49 \%$ \\
\hline Guatemala & $32.00 \%$ & $23.24 \%$ & $13.38 \%$ & $28.13 \%$ \\
\hline Guinea & $96.46 \%$ & $73.16 \%$ & $40.81 \%$ & $86.29 \%$ \\
\hline Guinea-Bissau & $92.60 \%$ & $70.64 \%$ & $42.30 \%$ & $83.07 \%$ \\
\hline Haiti & $109.17 \%$ & $81.07 \%$ & $47.11 \%$ & $97.17 \%$ \\
\hline Honduras & $21.27 \%$ & $16.21 \%$ & $9.82 \%$ & $19.25 \%$ \\
\hline India & $90.42 \%$ & $67.94 \%$ & $41.42 \%$ & $81.44 \%$ \\
\hline Iran, Islamic Rep. & $15.59 \%$ & $10.52 \%$ & $6.01 \%$ & $13.32 \%$ \\
\hline Jordan & $36.61 \%$ & $26.24 \%$ & $16.15 \%$ & $31.91 \%$ \\
\hline Kenya & $95.18 \%$ & $77.62 \%$ & $50.31 \%$ & $88.12 \%$ \\
\hline Lebanon & $26.73 \%$ & $19.52 \%$ & $13.17 \%$ & $23.77 \%$ \\
\hline Lesotho & $48.08 \%$ & $34.50 \%$ & $15.90 \%$ & $41.90 \%$ \\
\hline Liberia & $47.47 \%$ & $35.91 \%$ & $21.39 \%$ & $42.33 \%$ \\
\hline Libya & $63.11 \%$ & $43.88 \%$ & $23.20 \%$ & $54.62 \%$ \\
\hline Madagascar & $162.41 \%$ & $119.91 \%$ & $64.29 \%$ & $143.01 \%$ \\
\hline Malawi & $124.24 \%$ & $91.85 \%$ & $49.01 \%$ & $109.00 \%$ \\
\hline
\end{tabular}


medRxiv preprint doi: https://doi.org/10.1101/2020.08.23.20180299; this version posted August 25, 2020. The copyright holder for this preprint (which was not certified by peer review) is the author/funder, who has granted medRxiv a license to display the preprint in perpetuity.

\section{It is made available under a CC-BY-NC-ND 4.0 International license .}

\begin{tabular}{|c|c|c|c|c|}
\hline Mali & $112.89 \%$ & $80.98 \%$ & $41.16 \%$ & $98.27 \%$ \\
\hline Mauritania & $66.53 \%$ & $50.81 \%$ & $31.95 \%$ & $59.90 \%$ \\
\hline Mauritius & $51.80 \%$ & $35.77 \%$ & $22.65 \%$ & $44.85 \%$ \\
\hline Mexico & $41.70 \%$ & $30.10 \%$ & $19.29 \%$ & $36.94 \%$ \\
\hline Morocco & $35.59 \%$ & $26.48 \%$ & $16.55 \%$ & $32.03 \%$ \\
\hline Mozambique & $208.39 \%$ & $154.24 \%$ & $82.33 \%$ & $183.51 \%$ \\
\hline Namibia & $24.83 \%$ & $18.42 \%$ & $8.61 \%$ & $21.97 \%$ \\
\hline Nepal & $103.83 \%$ & $76.69 \%$ & $38.99 \%$ & $92.08 \%$ \\
\hline Nicaragua & $23.91 \%$ & $17.74 \%$ & $9.85 \%$ & $21.27 \%$ \\
\hline Niger & $104.35 \%$ & $74.27 \%$ & $37.63 \%$ & $89.96 \%$ \\
\hline Nigeria & $37.63 \%$ & $29.24 \%$ & $16.33 \%$ & $34.25 \%$ \\
\hline Pakistan & $68.03 \%$ & $49.49 \%$ & $28.11 \%$ & $59.39 \%$ \\
\hline Paraguay & $38.50 \%$ & $27.12 \%$ & $13.97 \%$ & $33.56 \%$ \\
\hline Peru & $55.06 \%$ & $38.39 \%$ & $19.64 \%$ & $47.65 \%$ \\
\hline Rwanda & $99.81 \%$ & $74.92 \%$ & $41.29 \%$ & $88.69 \%$ \\
\hline Sao Tome and Principe & $43.43 \%$ & $34.16 \%$ & $19.87 \%$ & $39.57 \%$ \\
\hline Senegal & $61.29 \%$ & $47.02 \%$ & $27.14 \%$ & $55.23 \%$ \\
\hline Sierra Leone & $37.86 \%$ & $28.70 \%$ & $17.12 \%$ & $33.82 \%$ \\
\hline South Africa & $41.70 \%$ & $31.27 \%$ & $18.61 \%$ & $37.90 \%$ \\
\hline Sri Lanka & $114.01 \%$ & $81.62 \%$ & $52.67 \%$ & $100.16 \%$ \\
\hline Sudan & $17.60 \%$ & $13.26 \%$ & $7.23 \%$ & $15.68 \%$ \\
\hline Syrian Arab Republic & $78.34 \%$ & $61.12 \%$ & $37.42 \%$ & $71.88 \%$ \\
\hline Tajikistan & $61.57 \%$ & $43.65 \%$ & $22.40 \%$ & $53.43 \%$ \\
\hline Tanzania & $132.50 \%$ & $100.81 \%$ & $55.90 \%$ & $119.13 \%$ \\
\hline Togo & $81.46 \%$ & $62.02 \%$ & $33.89 \%$ & $72.76 \%$ \\
\hline Tunisia & $26.91 \%$ & $19.19 \%$ & $11.26 \%$ & $23.77 \%$ \\
\hline Turkey & $72.68 \%$ & $52.66 \%$ & $34.64 \%$ & $64.39 \%$ \\
\hline Venezuela, RB & $9.61 \%$ & $7.02 \%$ & $4.44 \%$ & $8.63 \%$ \\
\hline Yemen, Rep. & $35.75 \%$ & $25.27 \%$ & $12.56 \%$ & $30.71 \%$ \\
\hline Zambia & $68.03 \%$ & $52.40 \%$ & $25.56 \%$ & $61.37 \%$ \\
\hline Zimbabwe & $46.04 \%$ & $37.58 \%$ & $35.55 \%$ & $43.51 \%$ \\
\hline
\end{tabular}


medRxiv preprint doi: https://doi.org/10.1101/2020.08.23.20180299; this version posted August 25, 2020. The copyright holder for this preprint (which was not certified by peer review) is the author/funder, who has granted medRxiv a license to display the preprint in perpetuity.

It is made available under a CC-BY-NC-ND 4.0 International license.

Table SR7: Health System Costs of COVID 19 Response as \% of government health spending

\begin{tabular}{|c|c|c|c|c|}
\hline Country & Scenario 1 & Scenario 4 & Scenario 14 & Scenario 49 \\
\hline Afghanistan & $1461.58 \%$ & $1079.37 \%$ & $609.33 \%$ & $1282.69 \%$ \\
\hline Algeria & $96.72 \%$ & $81.75 \%$ & $33.66 \%$ & $81.75 \%$ \\
\hline Angola & $131.58 \%$ & $121.02 \%$ & $50.32 \%$ & $121.02 \%$ \\
\hline Argentina & $53.71 \%$ & $47.42 \%$ & $24.33 \%$ & $47.42 \%$ \\
\hline Bangladesh & $984.27 \%$ & $883.78 \%$ & $419.89 \%$ & $883.78 \%$ \\
\hline Benin & $849.59 \%$ & $759.74 \%$ & $351.58 \%$ & $759.74 \%$ \\
\hline Bolivia & $52.17 \%$ & $46.49 \%$ & $17.12 \%$ & $46.49 \%$ \\
\hline Botswana & $87.93 \%$ & $77.14 \%$ & $35.75 \%$ & $77.14 \%$ \\
\hline Brazil & $80.84 \%$ & $70.48 \%$ & $32.38 \%$ & $70.48 \%$ \\
\hline Burkina Faso & $289.72 \%$ & $259.10 \%$ & $128.69 \%$ & $259.10 \%$ \\
\hline Burundi & $679.59 \%$ & $598.61 \%$ & $278.16 \%$ & $598.61 \%$ \\
\hline Cabo Verde & $79.91 \%$ & $70.07 \%$ & $32.13 \%$ & $70.07 \%$ \\
\hline Cambodia & $291.48 \%$ & $251.05 \%$ & $83.20 \%$ & $251.05 \%$ \\
\hline Cameroon & $532.62 \%$ & $481.16 \%$ & $223.68 \%$ & $481.16 \%$ \\
\hline Central African Republic & $1607.91 \%$ & $1422.83 \%$ & $739.81 \%$ & $1422.83 \%$ \\
\hline Chad & $712.01 \%$ & $617.48 \%$ & $258.53 \%$ & $617.48 \%$ \\
\hline Colombia & $112.68 \%$ & $98.29 \%$ & $44.17 \%$ & $98.29 \%$ \\
\hline Comoros & $553.08 \%$ & $493.60 \%$ & $228.65 \%$ & $493.60 \%$ \\
\hline Congo, Dem. Rep. & $1641.60 \%$ & $1451.34 \%$ & $598.70 \%$ & $1451.34 \%$ \\
\hline Congo, Rep. & $176.09 \%$ & $160.17 \%$ & $83.78 \%$ & $160.17 \%$ \\
\hline Costa Rica & $45.22 \%$ & $39.31 \%$ & $18.28 \%$ & $39.31 \%$ \\
\hline Cote d'Ivoire & $272.68 \%$ & $242.80 \%$ & $92.15 \%$ & $242.80 \%$ \\
\hline Cuba & $20.46 \%$ & $17.93 \%$ & $8.57 \%$ & $17.93 \%$ \\
\hline Djibouti & $197.29 \%$ & $179.69 \%$ & $86.95 \%$ & $179.69 \%$ \\
\hline Dominican Republic & $127.48 \%$ & $112.14 \%$ & $53.11 \%$ & $112.14 \%$ \\
\hline Ecuador & $74.65 \%$ & $65.88 \%$ & $31.15 \%$ & $65.88 \%$ \\
\hline Egypt, Arab Rep. & $170.63 \%$ & $150.12 \%$ & $63.26 \%$ & $150.12 \%$ \\
\hline El Salvador & $48.63 \%$ & $43.61 \%$ & $20.40 \%$ & $43.61 \%$ \\
\hline Equatorial Guinea & $266.82 \%$ & $243.24 \%$ & $115.92 \%$ & $243.24 \%$ \\
\hline Eswatini & $42.93 \%$ & $38.75 \%$ & $15.22 \%$ & $38.75 \%$ \\
\hline Ethiopia & $914.16 \%$ & $805.02 \%$ & $371.17 \%$ & $805.02 \%$ \\
\hline Gabon & $118.23 \%$ & $106.41 \%$ & $56.24 \%$ & $106.41 \%$ \\
\hline Gambia, The & $1345.47 \%$ & $1197.51 \%$ & $587.26 \%$ & $1197.51 \%$ \\
\hline Ghana & $210.35 \%$ & $188.62 \%$ & $72.25 \%$ & $188.62 \%$ \\
\hline Guatemala & $131.83 \%$ & $115.89 \%$ & $55.14 \%$ & $115.89 \%$ \\
\hline Guinea & $1174.62 \%$ & $1050.76 \%$ & $496.94 \%$ & $1050.76 \%$ \\
\hline Guinea-Bissau & $283.34 \%$ & $254.19 \%$ & $129.43 \%$ & $254.19 \%$ \\
\hline Haiti & $1008.94 \%$ & $898.02 \%$ & $435.37 \%$ & $898.02 \%$ \\
\hline Honduras & $67.20 \%$ & $60.80 \%$ & $31.03 \%$ & $60.80 \%$ \\
\hline India & $585.28 \%$ & $527.12 \%$ & $268.06 \%$ & $527.12 \%$ \\
\hline Iran, Islamic Rep. & $39.68 \%$ & $33.91 \%$ & $15.30 \%$ & $33.91 \%$ \\
\hline Jordan & $74.11 \%$ & $64.60 \%$ & $32.69 \%$ & $64.60 \%$ \\
\hline Kenya & $336.03 \%$ & $311.08 \%$ & $177.61 \%$ & $311.08 \%$ \\
\hline Lebanon & $67.74 \%$ & $60.24 \%$ & $33.38 \%$ & $60.24 \%$ \\
\hline Lesotho & $89.58 \%$ & $78.08 \%$ & $29.63 \%$ & $78.08 \%$ \\
\hline Liberia & $490.72 \%$ & $437.58 \%$ & $221.12 \%$ & $437.58 \%$ \\
\hline Libya & $99.72 \%$ & $86.30 \%$ & $36.66 \%$ & $86.30 \%$ \\
\hline Madagascar & $417.01 \%$ & $367.20 \%$ & $165.06 \%$ & $367.20 \%$ \\
\hline Malawi & $493.40 \%$ & $432.89 \%$ & $194.62 \%$ & $432.89 \%$ \\
\hline
\end{tabular}


medRxiv preprint doi: https://doi.org/10.1101/2020.08.23.20180299; this version posted August 25, 2020. The copyright holder for this preprint (which was not certified by peer review) is the author/funder, who has granted medRxiv a license to display the preprint in perpetuity. It is made available under a CC-BY-NC-ND 4.0 International license.

\begin{tabular}{|c|c|c|c|c|}
\hline Mali & $483.29 \%$ & $420.70 \%$ & $176.21 \%$ & $420.70 \%$ \\
\hline Mauritania & $273.93 \%$ & $246.63 \%$ & $131.56 \%$ & $246.63 \%$ \\
\hline Mauritius & $173.99 \%$ & $150.65 \%$ & $76.09 \%$ & $150.65 \%$ \\
\hline Mexico & $112.26 \%$ & $99.44 \%$ & $51.94 \%$ & $99.44 \%$ \\
\hline Morocco & $112.89 \%$ & $101.59 \%$ & $52.48 \%$ & $101.59 \%$ \\
\hline Mozambique & $420.65 \%$ & $370.42 \%$ & $166.19 \%$ & $370.42 \%$ \\
\hline Namibia & $43.22 \%$ & $38.24 \%$ & $14.98 \%$ & $38.24 \%$ \\
\hline Nepal & $868.62 \%$ & $770.34 \%$ & $326.22 \%$ & $770.34 \%$ \\
\hline Nicaragua & $51.54 \%$ & $45.85 \%$ & $21.22 \%$ & $45.85 \%$ \\
\hline Niger & $680.99 \%$ & $587.10 \%$ & $245.58 \%$ & $587.10 \%$ \\
\hline Nigeria & $506.26 \%$ & $460.77 \%$ & $219.63 \%$ & $460.77 \%$ \\
\hline Pakistan & $402.92 \%$ & $351.76 \%$ & $166.50 \%$ & $351.76 \%$ \\
\hline Paraguay & $102.82 \%$ & $89.61 \%$ & $37.32 \%$ & $89.61 \%$ \\
\hline Peru & $110.23 \%$ & $95.40 \%$ & $39.32 \%$ & $95.40 \%$ \\
\hline Rwanda & $313.36 \%$ & $278.46 \%$ & $129.63 \%$ & $278.46 \%$ \\
\hline Sao Tome and Principe & $124.55 \%$ & $113.46 \%$ & $56.99 \%$ & $113.46 \%$ \\
\hline Senegal & $269.12 \%$ & $242.51 \%$ & $119.16 \%$ & $242.51 \%$ \\
\hline Sierra Leone & $479.73 \%$ & $428.42 \%$ & $216.88 \%$ & $428.42 \%$ \\
\hline South Africa & $83.63 \%$ & $76.01 \%$ & $37.32 \%$ & $76.01 \%$ \\
\hline Sri Lanka & $397.17 \%$ & $348.94 \%$ & $183.49 \%$ & $348.94 \%$ \\
\hline Sudan & $157.07 \%$ & $139.90 \%$ & $64.52 \%$ & $139.90 \%$ \\
\hline Syrian Arab Republic & $173.02 \%$ & $158.74 \%$ & $82.63 \%$ & $158.74 \%$ \\
\hline Tajikistan & $357.52 \%$ & $310.26 \%$ & $130.06 \%$ & $310.26 \%$ \\
\hline Tanzania & $397.58 \%$ & $357.44 \%$ & $167.72 \%$ & $357.44 \%$ \\
\hline Togo & $611.16 \%$ & $545.92 \%$ & $254.28 \%$ & $545.92 \%$ \\
\hline Tunisia & $66.46 \%$ & $58.72 \%$ & $27.83 \%$ & $58.72 \%$ \\
\hline Turkey & $107.91 \%$ & $95.60 \%$ & $51.43 \%$ & $95.60 \%$ \\
\hline Venezuela, RB & $25.19 \%$ & $22.64 \%$ & $11.65 \%$ & $22.64 \%$ \\
\hline Yemen, Rep. & $635.34 \%$ & $545.82 \%$ & $223.14 \%$ & $545.82 \%$ \\
\hline Zambia & $199.20 \%$ & $179.70 \%$ & $74.84 \%$ & $179.70 \%$ \\
\hline Zimbabwe & $120.03 \%$ & $113.43 \%$ & $92.69 \%$ & $113.43 \%$ \\
\hline
\end{tabular}

Table SR8. Average Cost per Capita by Country Income Category (2019 US\$): Sensitivity Analysis on \% of symptomatic cases tested

\begin{tabular}{|c|c|c|c|c|}
\hline & Scenario 1 & Scenario 4 & Scenario 14 & Scenario 49 \\
\hline \multicolumn{5}{|c|}{ Average Cost per Capita by Country Income Category (2019 US\$): 20\% of Symptomatic Cases Tested } \\
\hline Low Income Countries (LIC) & 49.81 & 37.18 & 20.77 & 44. 12 \\
\hline Lower-Middle Income Countries (LMIC) & 62.64 & 47.09 & 28.00 & 56.30 \\
\hline Upper-Middle Income Countries (UMIC) & 85.05 & 60.62 & 36.41 & 74.74 \\
\hline \multicolumn{5}{|c|}{ Average Cost per Capita by Country Income Category (2019 US\$): 40\% of Symptomatic Cases Tested } \\
\hline Low Income Countries (LIC) & 51.00 & 38.07 & 21.26 & 45. 16 \\
\hline Lower-Middle Income Countries (LMIC) & 65.63 & 49.34 & 29.31 & 58.93 \\
\hline Upper-Middle Income Countries (UMIC) & 89.40 & 63.83 & 38.25 & 78.53 \\
\hline \multicolumn{5}{|c|}{ Average Cost per Capita by Country Income Category (2019 US\$): 60\% of Symptomatic Cases Tested } \\
\hline Low Income Countries (LIC) & 52.19 & 38.97 & 21.74 & 46.21 \\
\hline Lower-Middle Income Countries (LMIC) & 68.63 & 51.59 & 30.63 & 61.56 \\
\hline Upper-Middle Income Countries (UMIC) & 93.74 & 67.03 & 40.09 & 82.32 \\
\hline
\end{tabular}


medRxiv preprint doi: https://doi.org/10.1101/2020.08.23.20180299; this version posted August 25, 2020. The copyright holder for this preprint (which was not certified by peer review) is the author/funder, who has granted medRxiv a license to display the preprint in perpetuity.

\begin{abstract}
It is made available under a CC-BY-NC-ND 4.0 International license.
\end{abstract}

\begin{tabular}{|l|r|r|r|r|}
\hline \multicolumn{5}{|c|}{ Average Cost per Capita by Country Income Category (2019 US\$): 80\% of Symptomatic Cases Tested } \\
\hline Low Income Countries (LIC) & 53.39 & 39.86 & 22.23 & 47.25 \\
\hline Lower-Middle Income Countries (LMIC) & 71.62 & 53.85 & 31.94 & 64.19 \\
\hline Upper-Middle Income Countries (UMIC) & 98.09 & 70.24 & 41.93 & 86.11 \\
\hline \multicolumn{2}{|c|}{ Average Cost per Capita by Country Income Category (2019 US\$): 100\% of Symptomatic Cases Tested } \\
\hline Low Income Countries (LIC) & 54.58 & 40.75 & 22.72 & 48.30 \\
\hline Lower-Middle Income Countries (LMIC) & 74.62 & 56.10 & 33.25 & 66.82 \\
\hline Upper-Middle Income Countries (UMIC) & 102.44 & 73.44 & 43.77 & 89.90 \\
\hline
\end{tabular}


medRxiv preprint doi: https://doi.org/10.1101/2020.08.23.20180299; this version posted August 25, 2020. The copyright holder for this preprint (which was not certified by peer review) is the author/funder, who has granted medRxiv a license to display the preprint in perpetuity.

It is made available under a CC-BY-NC-ND 4.0 International license .

\section{Supplemental Material Methods}

Table of Contents

Supplement S1. Epidemiological model. 32

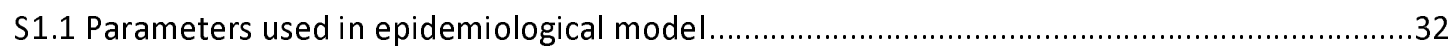

Figure S1.1.1 Flow diagram showing compartments and flows in the epidemiological model........32

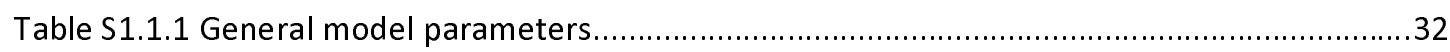

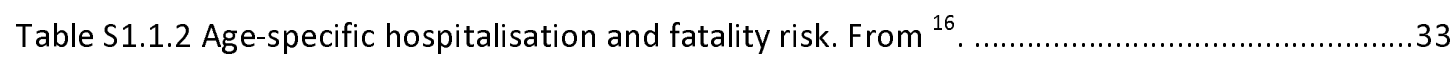

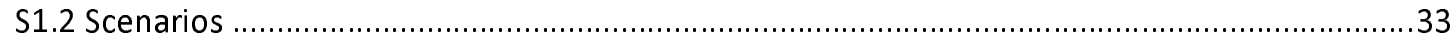

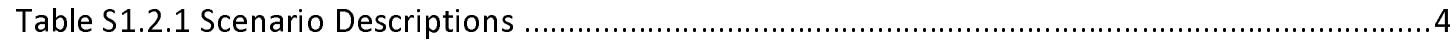

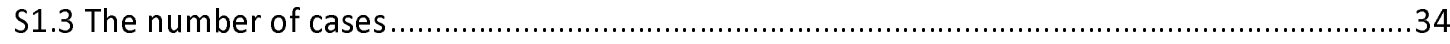

The total number of expected cases, deaths, and days of hospitalisation in ICU and general ward per country per scenario used in this study are in the table below (1). Table S1.3.1 Number of cases, deaths, and days of hospitalisation per country per scenario .......................................3

Supplemental S2. Parameters and process used in health resource and costing model..................46

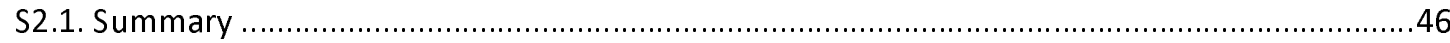

S2.2 Calculation of unit costs per activity for three base countries.....................................46

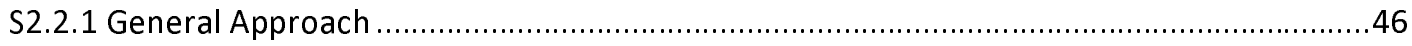

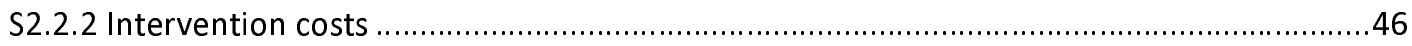

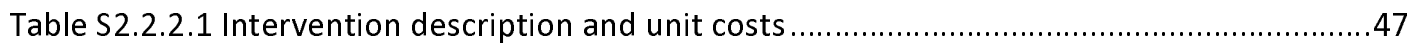

S.2.2.3. Defining Inputs, Costs per Input and Quantities of Inputs ....................................47

Table S2.2.3.1 Quantities and Unit Costs per Input per Activity per Country .........................48

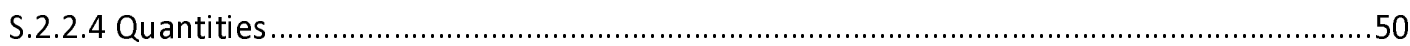

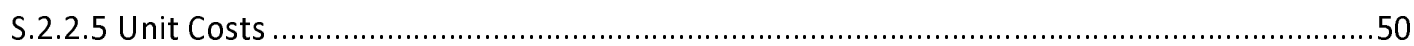

S2.2.4. Estimation of non-bed-day costs: Pakistan (ingredients costing) .............................50

S2.2.5. Estimation of non-bed-day costs: Ethiopia and South Africa (recently collected primary

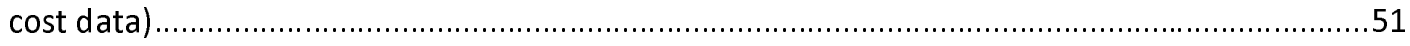

S2.2.6. Price adjustments and initial extrapolations .................... Error! Bookmark not defined.

Table S.2.2.6.1Inputs for which unit costs were partially or entirely extrapolated from Pakistan

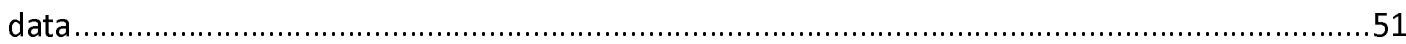

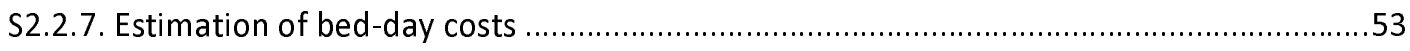

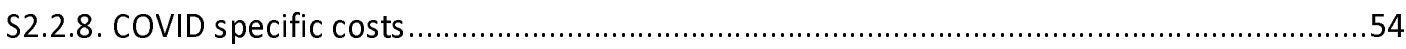

S2.3 Extrapolation of unit costs in base countries to calculate unit costs across 84 LICS, LMICs and

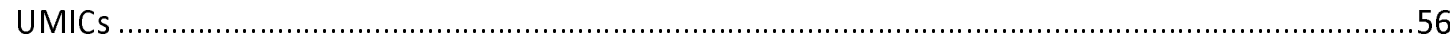

Table S2.3.1 Health worker earnings as a multiple of GDP per capita (Serje et al 2018)...............57 
medRxiv preprint doi: https://doi.org/10.1101/2020.08.23.20180299; this version posted August 25, 2020. The copyright holder for this preprint (which was not certified by peer review) is the author/funder, who has granted medRxiv a license to display the preprint in perpetuity. It is made available under a CC-BY-NC-ND 4.0 International license.

S2.4 Calculation of total costs per country using country-specific unit costs, modelled data on the number of cases, hospitalisations and deaths, as well as other epidemiological and economic assumptions

S2.5 Calculation of country-specific per capita costs, as well as per capita costs as a proportion of gross domestic product (GDP) per capita and various measures of health expenditure per capita.

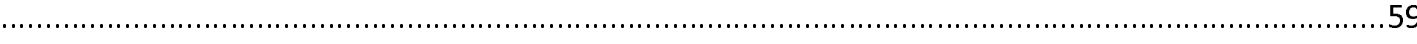

S.2.5.1 Total number of cases, ICU bed days, non-ICU bed days and deaths per country ...............60

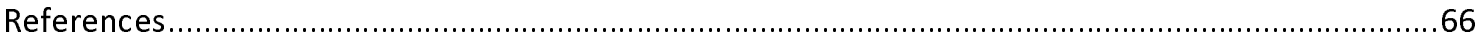


Supplement S1. Epidemiological model

\section{S1.1 Parameters used in epidemiological model}

Figure S1.1.1 Flow diagram showing compartments and flows in the epidemiological model.

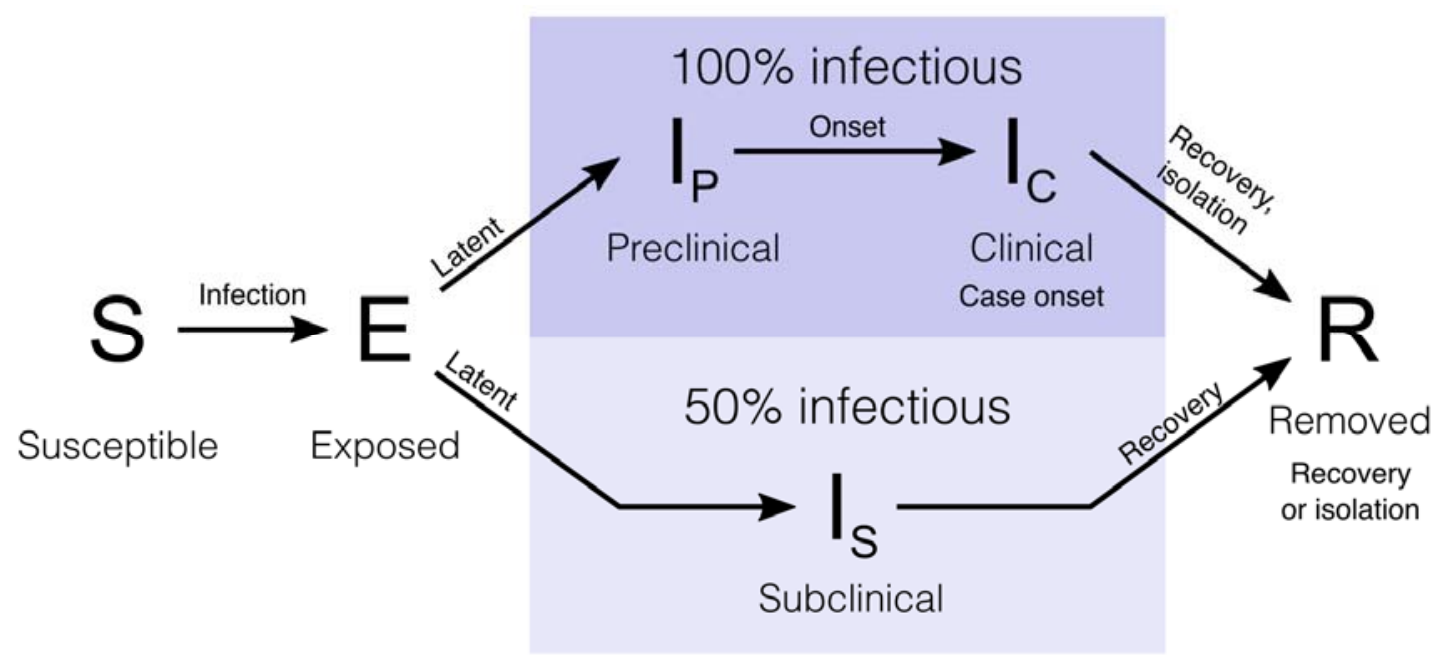

Table S1.1.1 General model parameters

\begin{tabular}{|c|c|c|c|}
\hline $\begin{array}{l}\text { Paramet } \\
\text { er }\end{array}$ & Description & Value & Reference \\
\hline$d_{E}$ & Latent period ( $E$ to $I_{P}$ and $E$ to $I_{S} ;$ days) & $\begin{array}{l}\text { Gamma distribution } \\
(\mu=4.0, k=4)\end{array}$ & 1 \\
\hline$d_{P}$ & $\begin{array}{l}\text { Duration of preclinical infectiousness } \\
\text { (I } I_{p} \text { to } I_{C} ; \text { days) }\end{array}$ & $\begin{array}{l}\text { Gamma distribution } \\
(\mu=2.4, \mathrm{k}=4)\end{array}$ & 1 \\
\hline$d_{C}$ & $\begin{array}{l}\text { Duration of clinical infectiousness (Ic } \\
\text { to } R \text {; days) }\end{array}$ & $\begin{array}{l}\text { Gamma distribution } \\
(\mu=3.2, \mathrm{k}=3.7)\end{array}$ & 2 \\
\hline \multirow[t]{3}{*}{$d_{S}$} & $\begin{array}{l}\text { Duration of subclinical infectiousness } \\
\text { (I } I_{s} \text { to } R \text {; days) }\end{array}$ & $\begin{array}{l}\text { Gamma distribution } \\
(\mu=7.0, k=4.0)\end{array}$ & Assumed \\
\hline & Incubation period (E to $I_{C}$; days) & $d_{E}+d_{P} ;$ mean 6.4 days & Derived \\
\hline & Serial interval (days) & $\begin{array}{l}d_{E}+\left(y_{i}\left(d_{P}+d_{C}\right)+\right. \\
\left.\left(1-y_{i}\right) d_{S}\right) / 2 ; \text { mean } \\
\text { approximately } 7 \text { days }\end{array}$ & Derived \\
\hline$u$ & Susceptibility to infection on contact & Calculated from $R_{0}$ & Derived \\
\hline$y_{i}$ & $\begin{array}{l}\text { Probability of clinical symptoms on } \\
\text { infection for age group } i\end{array}$ & $\begin{array}{l}\text { Estimated from case } \\
\text { distributions across } 6 \\
\text { countries }\end{array}$ & 3 \\
\hline$f$ & $\begin{array}{l}\text { Relative infectiousness of subclinical } \\
\text { cases }\end{array}$ & $50 \%$ & Assumed \\
\hline$c_{i j}$ & $\begin{array}{l}\text { Number of age- } j \text { individuals } \\
\text { contacted by an age- } i \text { individual per }\end{array}$ & $\begin{array}{l}\text { Country-specific contact } \\
\text { matrix }\end{array}$ & 4 \\
\hline
\end{tabular}




\begin{tabular}{|l|l|l|l|}
\hline & day & \\
\hline$N_{i}$ & Number of age- individuals & Demographic data & 5 \\
\hline$\Delta t$ & $\begin{array}{l}\text { Time step for discrete-time } \\
\text { simulation }\end{array}$ & 0.25 days & \\
\hline $\begin{array}{l}\text { Delay from onset to hospitalisation } \\
\text { (days) }\end{array}$ & $\begin{array}{l}\text { Gamma distribution } \\
(\mu=7.0, \mathrm{k}=5.0)\end{array}$ & \\
\hline $\begin{array}{l}\text { Duration of hospitalisation in non-ICU } \\
\text { bed, severe case (days) }\end{array}$ & $\begin{array}{l}\text { Gamma distribution } \\
(\mu=14.6, \mathrm{k}=5.0)\end{array}$ & 6 \\
\hline $\begin{array}{l}\text { Duration of hospitalisation in non-ICU } \\
\text { bed, critical case (before ICU bed; } \\
\text { days) }\end{array}$ & $\begin{array}{l}\text { Gamma distribution } \\
(\mu=6.0, \mathrm{k}=5.0)\end{array}$ & $7-13$ \\
\hline $\begin{array}{l}\text { Duration of hospitalisation in ICU } \\
\text { bed, critical case (after non-ICU bed; } \\
\text { days) }\end{array}$ & $\begin{array}{l}\text { Gamma distribution } \\
(\mu=9.6, \mathrm{k}=5.0)\end{array}$ & $7-13$ \\
\hline $\begin{array}{l}\text { Delay from onset to death (days) } \\
\text { Gamma distribution } \\
(\mu=22, \mathrm{k}=10)\end{array}$ & 14,15 \\
\hline
\end{tabular}

Table S1.1.2 Age-specific hospitalisation and fatality risk. From ${ }^{16}$.

$\begin{array}{llll}\text { Age group } & \text { Case-fatality risk } & \% \text { of cases hospitalised } & \% \text { of hospital patients needing ICU } \\ 0-9 & 0.00 \% & 0.0 \% & 30 \% \\ 1-10 & 0.09 \% & 0.8 \% & 30 \% \\ 20-29 & 0.10 \% & 0.8 \% & 30 \% \\ 30-39 & 0.12 \% & 1.0 \% & 30 \% \\ 40-49 & 0.23 \% & 1.9 \% & 30 \% \\ 50-59 & 0.68 \% & 5.4 \% & 30 \% \\ 60-69 & 1.87 \% & 15.1 \% & 30 \% \\ 70-79 & 4.14 \% & 33.3 \% & 30 \% \\ 80+ & 7.68 \% & 61.8 \% & 30 \%\end{array}$

\section{S1.2 Scenarios}

The epidemiological model uses data from 92 low- and middle-income countries. For each country, the model produces estimates on the number of cases, hospitalisations, number of days in hospital for severe cases (general ward) and critical cases (intensive care unit), and deaths for 57 distinct epidemiological scenarios [16].

For this study, four epidemiological scenarios were chosen out of the set of 57 possible scenarios. Scenario 1 represents an unmitigated epidemic. The other three scenarios were chosen because they represent a variety of plausible policy options (scenarios 4,14 and 49). Descriptions of the scenarios are presented below. 
Table S1.2.1 Scenario Descriptions

\begin{tabular}{|c|c|}
\hline Scenario 1 & Unmitigated epidemic \\
\hline Scenario 4 & $\begin{array}{l}\text { The whole population is covered in this intervention scenario. The intervention is } \\
\text { triggered by daily incidence reaching } 1 \text { per } 10,000 \text {. The intervention includes self- } \\
\text { isolation of symptomatic persons for duration of symptoms, modelled as an } \\
\text { additional reduction in contacts among symptomatic people of } 75 \% \text {. The } \\
\text { intervention includes distancing measures that reduce contacts at school by } 20 \\
\% \text {.The intervention includes distancing measures that reduce contacts at work by } \\
20 \% \text {.The intervention includes distancing measures that reduce contacts in other } \\
\text { settings by } 20 \% \text {.The intervention includes distancing measures that reduce } \\
\text { contacts in the home setting by } 0 \% \text {. There is no difference in intervention by age. }\end{array}$ \\
\hline Scenario 14 & $\begin{array}{l}\text { The whole population is covered in this intervention scenario. The intervention is } \\
\text { triggered by daily incidence reaching } 1 \text { per } 10,000 \text {. The intervention includes self- } \\
\text { isolation of symptomatic persons for duration of symptoms, modelled as an } \\
\text { additional reduction in contacts among symptomatic people of } 25 \% \text {. The } \\
\text { intervention includes distancing measures that reduce contacts at school by } 60 \\
\% \text {.The intervention includes distancing measures that reduce contacts at work by } \\
60 \% \text {.The intervention includes distancing measures that reduce contacts in other } \\
\text { settings by } 60 \% \text {.The intervention includes distancing measures that reduce } \\
\text { contacts in the home setting by } 0 \% \text {. There is no difference in intervention by age. }\end{array}$ \\
\hline Scenario 49 & $\begin{array}{l}\text { 49.The intervention is temporary lockdown which leads to } 100 \% \text { of the } \\
\text { population reducing their contacts through school, home, work and other settings } \\
\text { by } 100 \%, 0 \%, 37.5 \% \text { and } 37.5 \% \text { respectively for whilst in lockdown. Lockdown } \\
\text { occurs for the first } 30 \text { days. After lockdown is lifted } 100 \% \text { of the population } \\
\text { reducing their contacts through school, home, work and other settings by } 20 \%, 0 \\
\%, 20 \% \text { and } 20 \% \text { respectively for whilst not in lockdown }\end{array}$ \\
\hline
\end{tabular}

\section{S1.3 The number of cases}

The total number of expected cases, deaths, and days of hospitalisation in ICU and general ward per country per scenario used in this study are in the table below [16]. 
medRxiv preprint doi: https://doi.org/10.1101/2020.08.23.20180299; this version posted August 25, 2020. The copyright holder for this preprint (which was not certified by peer review) is the author/funder, who has granted medRxiv a license to display the preprint in perpetuity.

It is made available under a CC-BY-NC-ND 4.0 International license.

Table S1.3.1 Number of cases, deaths, and days of hospitalisation per country per scenario

\begin{tabular}{|c|c|c|c|c|c|}
\hline Country & Scenario & $\begin{array}{l}\text { Total Number of } \\
\text { Cases }\end{array}$ & $\begin{array}{l}\text { Total Number of } \\
\text { ICU bed days }\end{array}$ & $\begin{array}{l}\text { Total Number of } \\
\text { Non-ICU Bed Days }\end{array}$ & $\begin{array}{l}\text { Total Number of } \\
\text { Deaths }\end{array}$ \\
\hline Afghanistan & 1 & $12,218,383.50$ & $1,644,757.00$ & $3,068,969.00$ & $102,556.50$ \\
\hline Afghanistan & 4 & $9,128,640.00$ & $1,124,521.50$ & $2,096,065.00$ & $69,979.50$ \\
\hline Afghanistan & 14 & $5,057,916.50$ & $659,168.00$ & $1,228,499.50$ & $40,910.50$ \\
\hline Afghanistan & 49 & $10,687,758.50$ & $1,379,971.50$ & $2,571,195.50$ & $85,767.00$ \\
\hline Angola & 1 & $10,568,601.50$ & $1,337,285.50$ & $2,495,053.00$ & $82,996.50$ \\
\hline Angola & 4 & $7,951,658.50$ & $958,470.50$ & $1,790,227.00$ & $59,579.00$ \\
\hline Angola & 14 & $3,787,000.00$ & $480,525.00$ & $904,767.50$ & $29,918.00$ \\
\hline Angola & 49 & $9,243,635.50$ & $1,153,108.50$ & $2,151,983.50$ & $71,542.50$ \\
\hline United A rab Emirates & 1 & $3,070,769.50$ & $350,542.50$ & $651,357.50$ & $21,779.50$ \\
\hline United A rab Emirates & 4 & $2,177,762.50$ & $214,356.00$ & $402,281.50$ & $13,375.50$ \\
\hline United Arab Emirates & 14 & $1,024,344.50$ & $89,813.50$ & $167,419.00$ & $5,562.50$ \\
\hline United A rab Emirates & 49 & $2,602,624.00$ & $273,597.50$ & $513,621.00$ & $17,056.00$ \\
\hline Argentina & 1 & $16,773,844.50$ & $7,129,191.50$ & $13,331,842.50$ & $443,779.00$ \\
\hline Argentina & 4 & $12,167,825.00$ & $4,923,903.50$ & $9,183,093.00$ & $306,424.50$ \\
\hline Argentina & 14 & $7,206,744.00$ & $3,179,286.50$ & $5,930,434.50$ & $197,212.00$ \\
\hline Argentina & 49 & $14,559,346.00$ & $6,161,078.00$ & $11,508,389.50$ & $383,318.00$ \\
\hline Burundi & 1 & $3,825,576.00$ & $487,746.00$ & $912,820.50$ & $30,294.00$ \\
\hline Burundi & 4 & $2,875,191.50$ & $343,198.50$ & $643,374.00$ & $21,457.50$ \\
\hline Burundi & 14 & $1,563,193.50$ & $192,616.50$ & $359,761.00$ & $11,941.00$ \\
\hline Burundi & 49 & $3,360,357.50$ & $416,410.00$ & $783,449.00$ & $25,958.50$ \\
\hline Benin & 1 & $3,987,007.50$ & $610,334.00$ & $1,140,852.50$ & $37,966.50$ \\
\hline Benin & 4 & $3,027,030.50$ & $436,070.50$ & $813,543.50$ & $27,098.50$ \\
\hline Benin & 14 & $1,617,554.00$ & $243,858.00$ & $455,765.00$ & $15,180.50$ \\
\hline Benin & 49 & $3,517,493.00$ & $523,439.50$ & $980,737.50$ & $32,600.50$ \\
\hline Burkina Faso & 1 & $6,855,948.00$ & $954,394.50$ & $1,781,199.00$ & $59,384.50$ \\
\hline Burkina Faso & 4 & $5,231,105.00$ & $692,106.50$ & $1,290,564.50$ & $42,964.50$ \\
\hline Burkina Faso & 14 & $2,954,321.00$ & $421,062.50$ & $787,440.50$ & $26,089.50$ \\
\hline Burkina Faso & 49 & $6,035,205.50$ & $826,512.00$ & $1,546,412.50$ & $51,439.50$ \\
\hline Bangladesh & 1 & $57,227,126.50$ & $14,236,787.50$ & $26,575,364.00$ & $885,268.50$ \\
\hline Bangladesh & 4 & $43,270,144.00$ & $10,038,443.00$ & $18,753,926.00$ & $623,994.50$ \\
\hline Bangladesh & 14 & $23,696,468.50$ & $5,910,527.00$ & $11,038,186.50$ & $366,350.50$ \\
\hline Bangladesh & 49 & $50,407,176.00$ & $12,324,088.50$ & $23,001,526.50$ & $766,395.00$ \\
\hline Bahrain & 1 & $547,191.50$ & $80,485.50$ & $150,131.00$ & $5,024.50$ \\
\hline
\end{tabular}


medRxiv preprint doi: https://doi.org/10.1101/2020.08.23.20180299; this version posted August 25, 2020. The copyright holder for this preprint (which was not certified by peer review) is the author/funder, who has granted medRxiv a license to display the preprint in perpetuity.

It is made available under a CC-BY-NC-ND 4.0 International license.

\begin{tabular}{|c|c|c|c|c|c|}
\hline Bahrain & 4 & $391,589.50$ & $50,957.50$ & $94,643.50$ & $3,172.50$ \\
\hline Bahrain & 14 & $197,184.50$ & $23,694.50$ & $43,975.00$ & $1,460.00$ \\
\hline Bahrain & 49 & $468,646.00$ & $64,898.00$ & $121,082.00$ & $3,992.00$ \\
\hline Bolivia & 1 & $3,881,885.00$ & $929,770.50$ & $1,734,888.50$ & $57,656.00$ \\
\hline Bolivia & 4 & $2,855,102.00$ & $614,416.00$ & $1,146,287.00$ & $38,054.00$ \\
\hline Bolivia & 14 & $1,246,487.00$ & $279,998.50$ & $519,996.50$ & $17,368.00$ \\
\hline Bolivia & 49 & $3,364,052.00$ & $778,136.00$ & $1,449,732.50$ & $48,330.00$ \\
\hline Brazil & 1 & $76,815,441.00$ & $27,418,042.50$ & $51,183,618.50$ & $1,705,997.00$ \\
\hline Brazil & 4 & $56,285,085.00$ & $18,283,101.50$ & $34,119,544.00$ & $1,139,191.50$ \\
\hline Brazil & 14 & $30,752,013.00$ & $10,628,491.00$ & $19,851,592.50$ & $658,619.00$ \\
\hline Brazil & 49 & $66,729,481.50$ & $23,242,138.50$ & $43,395,705.00$ & $1,445,675.00$ \\
\hline Botswana & 1 & $765,573.00$ & $159,460.50$ & $296,628.50$ & $9,918.00$ \\
\hline Botswana & 4 & $565,035.50$ & $107,208.00$ & $199,542.00$ & $6,659.50$ \\
\hline Botswana & 14 & $305,081.50$ & $61,841.00$ & $114,201.00$ & $3,788.50$ \\
\hline Botswana & 49 & $662,701.50$ & $134,417.50$ & $250,989.00$ & $8,333.50$ \\
\hline Central African Republic & 1 & $1,542,576.00$ & $227,256.00$ & $422,653.00$ & $14,107.50$ \\
\hline Central African Republic & 4 & $1,168,850.50$ & $158,951.50$ & $297,690.50$ & $9,899.50$ \\
\hline Central African Republic & 14 & $696,606.50$ & $102,635.00$ & $192,340.00$ & $6,435.00$ \\
\hline Central African Republic & 49 & $1,353,904.00$ & $194,598.50$ & $363,300.50$ & $12,130.00$ \\
\hline Chile & 1 & $7,194,690.00$ & $3,077,838.50$ & $5,737,576.50$ & $191,424.00$ \\
\hline Chile & 4 & $5,194,730.50$ & $2,043,884.50$ & $3,820,076.00$ & $126,898.00$ \\
\hline Chile & 14 & $3,037,288.00$ & $1,252,044.50$ & $2,330,419.00$ & $77,706.50$ \\
\hline Chile & 49 & $6,244,492.50$ & $2,596,331.50$ & $4,850,755.50$ & $161,525.00$ \\
\hline Cote d'Ivoire & 1 & $8,535,011.50$ & $1,316,578.00$ & $2,457,105.50$ & $82,052.50$ \\
\hline Cote d'Ivoire & 4 & $6,298,091.50$ & $916,128.00$ & $1,708,562.00$ & $57,155.50$ \\
\hline Cote d'Ivoire & 14 & $2,781,975.00$ & $429,891.00$ & $803,017.00$ & $26,661.00$ \\
\hline Cote d'Ivoire & 49 & $7,403,108.50$ & $1,119,330.00$ & $2,085,969.00$ & $69,716.00$ \\
\hline Cameroon & 1 & $8,729,175.00$ & $1,249,335.00$ & $2,335,084.00$ & $77,781.00$ \\
\hline Cameroon & 4 & $6,604,562.00$ & $889,631.00$ & $1,661,757.00$ & $55,432.00$ \\
\hline Cameroon & 14 & $3,552,194.00$ & $503,335.50$ & $939,579.50$ & $31,264.50$ \\
\hline Cameroon & 49 & $7,690,769.50$ & $1,078,672.50$ & $2,014,896.00$ & $67,062.00$ \\
\hline Congo, Dem. Rep. & 1 & $28,914,534.50$ & $4,131,602.50$ & $7,713,653.50$ & $257,149.00$ \\
\hline Congo, Dem. Rep. & 4 & $21,729,089.00$ & $2,925,815.50$ & $5,459,743.00$ & $181,724.50$ \\
\hline Congo, Dem. Rep. & 14 & $10,339,604.50$ & $1,480,493.00$ & $2,755,736.00$ & $91,222.50$ \\
\hline Congo, Dem. Rep. & 49 & $25,274,207.00$ & $3,537,679.50$ & $6,601,660.50$ & $219,628.00$ \\
\hline Congo, Rep. & 1 & $1,819,209.50$ & $284,222.00$ & $531,531.00$ & $17,714.00$ \\
\hline Congo, Rep. & 4 & $1,380,686.00$ & $201,095.00$ & $376,298.50$ & $12,564.00$ \\
\hline Congo, Rep. & 14 & $828,849.00$ & $128,923.50$ & $241,162.00$ & $8,084.50$ \\
\hline
\end{tabular}


medRxiv preprint doi: https://doi.org/10.1101/2020.08.23.20180299; this version posted August 25, 2020. The copyright holder for this preprint (which was not certified by peer review) is the author/funder, who has granted medRxiv a license to display the preprint in perpetuity.

It is made available under a CC-BY-NC-ND 4.0 International license.

\begin{tabular}{|c|c|c|c|c|c|}
\hline Congo, Rep. & 49 & $1,603,300.00$ & $245,240.00$ & $457,933.50$ & $15,290.00$ \\
\hline Colombia & 1 & $18,341,207.00$ & $6,156,013.50$ & $11,493,003.50$ & $382,744.50$ \\
\hline Colombia & 4 & $13,426,478.00$ & $4,130,466.00$ & $7,696,118.00$ & $256,667.50$ \\
\hline Colombia & 14 & $7,192,837.50$ & $2,342,406.50$ & $4,372,127.00$ & $144,932.50$ \\
\hline Colombia & 49 & $15,964,752.50$ & $5,231,315.00$ & $9,754,859.00$ & $325,054.00$ \\
\hline Comoros & 1 & $290,557.00$ & $47,011.00$ & $87,852.50$ & $2,911.50$ \\
\hline Comoros & 4 & $215,334.50$ & $32,916.00$ & $61,230.50$ & $2,047.00$ \\
\hline Comoros & 14 & $114,051.50$ & $18,550.50$ & $34,354.00$ & $1,129.00$ \\
\hline Comoros & 49 & $252,887.00$ & $40,321.50$ & $75,628.00$ & $2,512.00$ \\
\hline Cabo Verde & 1 & $181,545.00$ & $41,378.50$ & $77,437.00$ & $2,565.50$ \\
\hline Cabo Verde & 4 & $128,006.00$ & $26,548.00$ & $49,137.00$ & $1,638.00$ \\
\hline Cabo Verde & 14 & $67,260.50$ & $14,657.00$ & $27,550.00$ & 904.50 \\
\hline Cabo Verde & 49 & $153,456.00$ & $33,984.50$ & $63,202.50$ & $2,102.00$ \\
\hline Costa Rica & 1 & $1,822,572.50$ & $652,845.00$ & $1,218,193.50$ & $40,619.00$ \\
\hline Costa Rica & 4 & $1,326,970.50$ & $428,626.50$ & $799,723.50$ & $26,658.50$ \\
\hline Costa Rica & 14 & $745,696.00$ & $252,692.50$ & $470,210.00$ & $15,640.50$ \\
\hline Costa Rica & 49 & $1,578,679.00$ & $549,464.00$ & $1,023,618.50$ & $34,173.50$ \\
\hline Cuba & 1 & $4,423,054.50$ & $2,120,624.00$ & $3,955,835.50$ & $131,915.50$ \\
\hline Cuba & 4 & $3,216,773.00$ & $1,414,337.00$ & $2,640,812.00$ & $87,972.00$ \\
\hline Cuba & 14 & $1,810,327.00$ & $857,054.50$ & $1,601,201.00$ & $53,185.50$ \\
\hline Cuba & 49 & $3,831,103.00$ & $1,804,660.00$ & $3,363,770.00$ & $111,899.50$ \\
\hline Djibouti & 1 & $343,723.00$ & $73,381.50$ & $136,983.50$ & $4,544.00$ \\
\hline Djibouti & 4 & $261,053.50$ & $51,856.50$ & $97,124.00$ & $3,227.50$ \\
\hline Djibouti & 14 & $142,168.00$ & $30,426.50$ & $55,879.50$ & $1,876.50$ \\
\hline Djibouti & 49 & $302,937.00$ & $63,302.00$ & $118,347.50$ & $3,926.00$ \\
\hline Dominican Republic & 1 & $3,807,776.50$ & $1,127,809.00$ & $2,105,556.50$ & $70,140.00$ \\
\hline Dominican Republic & 4 & $2,808,434.50$ & $765,172.00$ & $1,422,811.50$ & $47,526.00$ \\
\hline Dominican Republic & 14 & $1,562,725.00$ & $456,252.50$ & $850,089.00$ & $28,342.50$ \\
\hline Dominican Republic & 49 & $3,315,475.00$ & $964,871.00$ & $1,796,265.00$ & $59,882.00$ \\
\hline Algeria & 1 & $14,034,752.50$ & $3,466,814.50$ & $6,462,517.50$ & $215,452.50$ \\
\hline Algeria & 4 & $9,901,969.50$ & $2,171,943.50$ & $4,050,558.50$ & $135,157.00$ \\
\hline Algeria & 14 & $5,054,134.00$ & $1,144,758.00$ & $2,139,220.50$ & $70,839.50$ \\
\hline Algeria & 49 & $11,937,307.00$ & $2,835,057.50$ & $5,295,541.50$ & $175,988.50$ \\
\hline Ecuador & 1 & $6,238,253.50$ & $1,828,486.50$ & $3,416,054.50$ & $113,769.50$ \\
\hline Ecuador & 4 & $4,681,265.50$ & $1,256,086.50$ & $2,337,135.50$ & $77,960.00$ \\
\hline Ecuador & 14 & $2,586,022.00$ & $739,506.00$ & $1,376,652.00$ & $45,789.00$ \\
\hline Ecuador & 49 & $5,480,200.00$ & $1,567,059.00$ & $2,923,489.50$ & $97,213.50$ \\
\hline Egypt, Arab Rep. & 1 & $32,689,306.00$ & $7,149,997.50$ & $13,352,307.50$ & $444,818.00$ \\
\hline
\end{tabular}


medRxiv preprint doi: https://doi.org/10.1101/2020.08.23.20180299; this version posted August 25, 2020. The copyright holder for this preprint (which was not certified by peer review) is the author/funder, who has granted medRxiv a license to display the preprint in perpetuity.

It is made available under a CC-BY-NC-ND 4.0 International license.

\begin{tabular}{|c|c|c|c|c|c|}
\hline Egypt, Arab Rep. & 4 & $23,251,249.50$ & $4,594,336.00$ & $8,576,020.00$ & $285,393.00$ \\
\hline Egypt, Arab Rep. & 14 & $11,840,378.50$ & $2,461,238.50$ & $4,607,142.50$ & $152,674.00$ \\
\hline Egypt, Arab Rep. & 49 & $27,975,740.50$ & $5,946,558.50$ & $11,123,014.50$ & $370,198.00$ \\
\hline Eritrea & 1 & $1,155,784.50$ & $209,554.50$ & $391,035.00$ & $13,020.50$ \\
\hline Eritrea & 4 & $871,719.50$ & $146,793.00$ & $273,048.00$ & $9,126.00$ \\
\hline Eritrea & 14 & $463,730.00$ & $85,068.00$ & $156,997.50$ & $5,297.00$ \\
\hline Eritrea & 49 & $1,011,765.50$ & $180,018.50$ & $336,576.50$ & $11,223.50$ \\
\hline Ethiopia & 1 & $37,424,354.50$ & $6,123,829.50$ & $11,438,760.00$ & $380,821.00$ \\
\hline Ethiopia & 4 & $28,272,226.50$ & $4,318,604.50$ & $8,066,304.00$ & $268,718.00$ \\
\hline Ethiopia & 14 & $14,996,879.50$ & $2,457,011.50$ & $4,593,451.50$ & $152,670.50$ \\
\hline Ethiopia & 49 & $32,804,209.50$ & $5,277,772.50$ & $9,861,570.50$ & $328,539.50$ \\
\hline Gabon & 1 & $752,439.50$ & $127,913.00$ & $239,230.50$ & $7,959.50$ \\
\hline Gabon & 4 & $572,425.50$ & $89,835.00$ & $168,146.00$ & $5,614.00$ \\
\hline Gabon & 14 & $347,308.00$ & $58,094.50$ & $109,709.00$ & $3,636.00$ \\
\hline Gabon & 49 & $664,127.50$ & $110,378.00$ & $206,161.50$ & $6,867.50$ \\
\hline Ghana & 1 & $10,378,959.00$ & $1,826,469.00$ & $3,406,631.50$ & $113,607.50$ \\
\hline Ghana & 4 & $7,713,893.50$ & $1,273,479.00$ & $2,379,059.00$ & $79,389.50$ \\
\hline Ghana & 14 & $3,423,372.00$ & $602,269.50$ & $1,120,060.00$ & $37,243.50$ \\
\hline Ghana & 49 & $9,039,576.00$ & $1,557,543.50$ & $2,901,100.00$ & $96,752.50$ \\
\hline Guinea & 1 & $4,258,486.00$ & $700,272.50$ & $1,311,263.50$ & $43,626.50$ \\
\hline Guinea & 4 & $3,197,000.00$ & $509,156.50$ & $950,868.50$ & $31,664.00$ \\
\hline Guinea & 14 & $1,702,114.50$ & $304,197.50$ & $565,934.00$ & $18,715.50$ \\
\hline Guinea & 49 & $3,718,928.00$ & $614,330.50$ & $1,144,140.00$ & $38,187.50$ \\
\hline Gambia, The & 1 & $787,667.00$ & $120,449.50$ & $224,341.50$ & $7,490.50$ \\
\hline Gambia, The & 4 & $592,870.50$ & $88,337.00$ & $163,947.00$ & $5,442.50$ \\
\hline Gambia, The & 14 & $324,941.50$ & $53,736.50$ & $101,076.00$ & $3,349.50$ \\
\hline Gambia, The & 49 & $686,599.00$ & $104,927.00$ & $196,707.00$ & $6,559.00$ \\
\hline Guinea-Bissau & 1 & $646,616.00$ & $100,305.50$ & $186,821.00$ & $6,207.50$ \\
\hline Guinea-Bissau & 4 & $490,671.00$ & $72,272.00$ & $135,491.00$ & $4,505.50$ \\
\hline Guinea-Bissau & 14 & $284,320.50$ & $45,433.50$ & $83,768.50$ & $2,782.00$ \\
\hline Guinea-Bissau & 49 & $569,959.50$ & $87,200.50$ & $163,160.00$ & $5,405.50$ \\
\hline Equatorial Guinea & 1 & $471,118.00$ & $62,021.00$ & $116,073.50$ & $3,858.00$ \\
\hline Equatorial Guinea & 4 & $359,112.50$ & $44,812.00$ & $83,461.50$ & $2,771.50$ \\
\hline Equatorial Guinea & 14 & $194,873.50$ & $25,338.00$ & $47,642.00$ & $1,593.50$ \\
\hline Equatorial Guinea & 49 & $416,196.50$ & $53,836.00$ & $100,579.00$ & $3,348.50$ \\
\hline Guatemala & 1 & $5,931,816.00$ & $1,188,377.50$ & $2,215,138.50$ & $73,895.00$ \\
\hline Guatemala & 4 & $4,445,955.00$ & $809,410.50$ & $1,509,951.00$ & $50,397.50$ \\
\hline Guatemala & 14 & $2,471,211.50$ & $477,647.50$ & $888,300.00$ & $29,575.50$ \\
\hline
\end{tabular}


medRxiv preprint doi: https://doi.org/10.1101/2020.08.23.20180299; this version posted August 25, 2020. The copyright holder for this preprint (which was not certified by peer review) is the author/funder, who has granted medRxiv a license to display the preprint in perpetuity.

It is made available under a CC-BY-NC-ND 4.0 International license.

\begin{tabular}{|c|c|c|c|c|c|}
\hline Guatemala & 49 & $5,200,461.00$ & $1,004,988.50$ & $1,873,662.50$ & $62,445.50$ \\
\hline Honduras & 1 & $3,368,666.00$ & $711,831.50$ & $1,328,438.50$ & $44,068.00$ \\
\hline Honduras & 4 & $2,554,279.00$ & $498,194.50$ & $928,828.50$ & $30,884.00$ \\
\hline Honduras & 14 & $1,508,914.50$ & $311,765.50$ & $579,275.50$ & $19,367.50$ \\
\hline Honduras & 49 & $2,966,095.00$ & $611,292.00$ & $1,145,329.00$ & $38,068.50$ \\
\hline Haiti & 1 & $3,844,733.00$ & $864,007.50$ & $1,611,689.00$ & $53,684.00$ \\
\hline Haiti & 4 & $2,891,883.50$ & $602,645.50$ & $1,127,804.50$ & $37,538.50$ \\
\hline Haiti & 14 & $1,628,745.00$ & $363,202.00$ & $678,225.00$ & $22,680.00$ \\
\hline Haiti & 49 & $3,378,437.50$ & $746,954.00$ & $1,392,144.50$ & $46,506.50$ \\
\hline India & 1 & $485,771,554.00$ & $134,139,960.00$ & $250,304,012.50$ & $8,338,437.50$ \\
\hline India & 4 & $365,933,321.50$ & $93,048,830.00$ & $173,656,181.50$ & $5,785,606.50$ \\
\hline India & 14 & $217,279,232.00$ & $58,353,362.00$ & $108,949,367.00$ & $3,620,179.00$ \\
\hline India & 49 & $427,295,820.50$ & $115,025,840.50$ & $214,808,061.50$ & $7,154,678.00$ \\
\hline Iran, Islamic Rep. & 1 & $27,824,797.00$ & $7,261,103.00$ & $13,561,427.50$ & $451,739.50$ \\
\hline Iran, Islamic Rep. & 4 & $19,593,066.00$ & $4,553,642.50$ & $8,499,919.50$ & $282,846.00$ \\
\hline Iran, Islamic Rep. & 14 & $11,174,742.50$ & $2,592,113.50$ & $4,837,163.50$ & $161,457.50$ \\
\hline Iran, Islamic Rep. & 49 & $23,673,028.50$ & $5,957,133.50$ & $11,119,019.00$ & $370,270.00$ \\
\hline Iraq & 1 & $13,378,016.00$ & $2,160,705.50$ & $4,036,576.50$ & $134,703.50$ \\
\hline Iraq & 4 & $10,055,987.00$ & $1,513,885.00$ & $2,832,294.00$ & $94,396.50$ \\
\hline Iraq & 14 & $5,444,675.50$ & $850,809.00$ & $1,586,860.50$ & $52,768.00$ \\
\hline Iraq & 49 & $11,745,266.50$ & $1,852,021.00$ & $3,459,984.50$ & $115,381.50$ \\
\hline Israel & 1 & $3,220,231.50$ & $1,398,523.50$ & $2,612,412.50$ & $86,885.00$ \\
\hline Israel & 4 & $2,318,309.00$ & $960,045.00$ & $1,794,150.50$ & $59,726.50$ \\
\hline Israel & 14 & $1,417,351.00$ & $644,261.00$ & $1,206,315.50$ & $39,883.00$ \\
\hline Israel & 49 & $2,790,877.00$ & $1,206,535.00$ & $2,251,874.50$ & $74,860.00$ \\
\hline Jordan & 1 & $3,230,120.50$ & $545,182.00$ & $1,014,537.00$ & $33,793.00$ \\
\hline Jordan & 4 & $2,403,684.50$ & $360,587.00$ & $671,528.00$ & $22,395.50$ \\
\hline Jordan & 14 & $1,441,001.50$ & $226,240.50$ & $421,434.00$ & $14,019.50$ \\
\hline Jordan & 49 & $2,802,081.50$ & $455,065.50$ & $851,418.00$ & $28,348.50$ \\
\hline Kenya & 1 & $17,932,717.50$ & $3,334,486.00$ & $6,220,435.00$ & $207,231.00$ \\
\hline Kenya & 4 & $13,972,380.00$ & $2,685,062.50$ & $5,015,623.00$ & $166,727.00$ \\
\hline Kenya & 14 & $8,920,886.50$ & $1,777,160.50$ & $3,304,638.00$ & $110,213.50$ \\
\hline Kenya & 49 & $15,940,230.00$ & $3,027,794.00$ & $5,654,038.00$ & $188,428.00$ \\
\hline Cambodia & 1 & $5,249,630.00$ & $1,075,358.00$ & $2,009,206.50$ & $67,003.00$ \\
\hline Cambodia & 4 & $3,735,696.50$ & $699,366.00$ & $1,307,951.50$ & $43,432.50$ \\
\hline Cambodia & 14 & $1,491,662.00$ & $285,706.00$ & $532,484.00$ & $17,649.50$ \\
\hline Cambodia & 49 & $4,455,701.50$ & $883,329.50$ & $1,656,497.50$ & $55,016.50$ \\
\hline Kuwait & 1 & $1,365,100.50$ & $250,497.00$ & $468,191.50$ & $15,520.50$ \\
\hline
\end{tabular}


medRxiv preprint doi: https://doi.org/10.1101/2020.08.23.20180299; this version posted August 25, 2020. The copyright holder for this preprint (which was not certified by peer review) is the author/funder, who has granted medRxiv a license to display the preprint in perpetuity.

It is made available under a CC-BY-NC-ND 4.0 International license.

\begin{tabular}{|c|c|c|c|c|c|}
\hline Kuwait & 4 & $927,501.50$ & $153,854.00$ & $289,037.50$ & $9,618.00$ \\
\hline Kuwait & 14 & $463,836.00$ & $72,077.00$ & $134,599.00$ & $4,499.00$ \\
\hline Kuwait & 49 & $1,135,226.00$ & $198,574.50$ & $372,262.50$ & $12,352.50$ \\
\hline Lebanon & 1 & $2,473,141.50$ & $849,633.00$ & $1,578,978.00$ & $52,778.00$ \\
\hline Lebanon & 4 & $1,838,520.50$ & $596,389.00$ & $1,111,151.00$ & $36,992.00$ \\
\hline Lebanon & 14 & $1,166,531.50$ & $410,943.00$ & $767,150.00$ & $25,627.50$ \\
\hline Lebanon & 49 & $2,165,967.50$ & $736,594.00$ & $1,374,880.00$ & $45,888.00$ \\
\hline Liberia & 1 & $1,679,614.00$ & $288,953.00$ & $537,754.50$ & $17,919.00$ \\
\hline Liberia & 4 & $1,277,408.00$ & $204,826.00$ & $383,901.50$ & $12,778.00$ \\
\hline Liberia & 14 & $740,784.00$ & $127,259.00$ & $237,192.00$ & $7,905.00$ \\
\hline Liberia & 49 & $1,478,591.00$ & $249,999.00$ & $464,731.00$ & $15,427.50$ \\
\hline Libya & 1 & $2,278,126.50$ & $480,466.00$ & $897,001.00$ & $29,872.50$ \\
\hline Libya & 4 & $1,632,674.50$ & $312,685.50$ & $585,736.00$ & $19,495.50$ \\
\hline Libya & 14 & $836,827.00$ & $167,080.50$ & $312,036.00$ & $10,330.00$ \\
\hline Libya & 49 & $1,947,756.50$ & $401,240.50$ & $748,905.50$ & $24,938.00$ \\
\hline Sri Lanka & 1 & $8,020,343.00$ & $3,408,469.50$ & $6,355,545.00$ & $211,681.00$ \\
\hline Sri Lanka & 4 & $5,916,153.00$ & $2,373,553.00$ & $4,436,366.50$ & $147,651.50$ \\
\hline Sri Lanka & 14 & $3,594,781.50$ & $1,558,313.00$ & $2,903,474.50$ & $96,594.00$ \\
\hline Sri Lanka & 49 & $7,016,699.00$ & $2,945,770.00$ & $5,508,073.00$ & $183,971.50$ \\
\hline Lesotho & 1 & $696,741.00$ & $145,159.00$ & $271,320.50$ & $9,016.50$ \\
\hline Lesotho & 4 & $508,495.00$ & $95,194.00$ & $178,041.00$ & $5,963.00$ \\
\hline Lesotho & 14 & $229,199.00$ & $44,271.50$ & $82,771.50$ & $2,796.00$ \\
\hline Lesotho & 49 & $600,007.00$ & $121,390.50$ & $224,865.00$ & $7,490.00$ \\
\hline Morocco & 1 & $13,242,616.50$ & $4,386,423.50$ & $8,186,000.00$ & $272,409.00$ \\
\hline Morocco & 4 & $9,819,052.50$ & $3,059,153.50$ & $5,703,002.00$ & $190,014.00$ \\
\hline Morocco & 14 & $5,899,932.00$ & $1,973,055.50$ & $3,680,791.50$ & $122,030.50$ \\
\hline Morocco & 49 & $11,587,125.00$ & $3,784,741.00$ & $7,071,203.00$ & $235,508.50$ \\
\hline Madagascar & 1 & $9,150,387.00$ & $1,448,349.50$ & $2,707,202.50$ & $90,125.50$ \\
\hline Madagascar & 4 & $6,782,766.00$ & $1,015,311.50$ & $1,897,325.50$ & $63,300.50$ \\
\hline Madagascar & 14 & $3,564,206.50$ & $563,427.50$ & $1,049,184.00$ & $35,009.50$ \\
\hline Madagascar & 49 & $7,966,524.50$ & $1,244,623.50$ & $2,321,257.00$ & $77,440.00$ \\
\hline Mexico & 1 & $46,024,528.50$ & $13,801,739.50$ & $25,788,652.50$ & $858,167.00$ \\
\hline Mexico & 4 & $34,417,215.50$ & $9,459,831.00$ & $17,652,017,50$ & $588,526.00$ \\
\hline Mexico & 14 & $21,097,465.50$ & $6,179,136.00$ & $11,554,982.50$ & $384,123.00$ \\
\hline Mexico & 49 & $40,449,264.50$ & $11,850,318.50$ & $22,128,971.50$ & $737,192.50$ \\
\hline Mali & 1 & $6,261,538.50$ & $750,361.50$ & $1,399,912.50$ & $46,770.00$ \\
\hline Mali & 4 & $4,479,076.50$ & $497,581.50$ & $928,693.00$ & $30,937.00$ \\
\hline Mali & 14 & $2,225,009.00$ & $265,778.50$ & $494,221.50$ & $16,416.00$ \\
\hline
\end{tabular}


medRxiv preprint doi: https://doi.org/10.1101/2020.08.23.20180299; this version posted August 25, 2020. The copyright holder for this preprint (which was not certified by peer review) is the author/funder, who has granted medRxiv a license to display the preprint in perpetuity.

It is made available under a CC-BY-NC-ND 4.0 International license.

\begin{tabular}{|c|c|c|c|c|c|}
\hline Mali & 49 & $5,359,818.00$ & $623,713.50$ & $1,164,621.00$ & $38,817.00$ \\
\hline Mozambique & 1 & $10,132,075.50$ & $1,434,436.00$ & $2,675,563.50$ & $89,192.00$ \\
\hline Mozambique & 4 & $7,529,591.00$ & $1,000,297.00$ & $1,869,916.50$ & $62,413.00$ \\
\hline Mozambique & 14 & $3,939,631.00$ & $555,317.50$ & $1,035,085.50$ & $34,251.00$ \\
\hline Mozambique & 49 & $8,820,156.50$ & $1,230,586.50$ & $2,293,159.50$ & $76,324.00$ \\
\hline Mauritania & 1 & $1,535,219.00$ & $254,585.50$ & $475,932.50$ & $15,814.50$ \\
\hline Mauritania & 4 & $1,165,275.50$ & $182,691.50$ & $339,829.50$ & $11,333.00$ \\
\hline Mauritania & 14 & $707,749.50$ & $121,283.50$ & $227,135.00$ & $7,523.50$ \\
\hline Mauritania & 49 & $1,353,942.00$ & $221,412.00$ & $412,271.50$ & $13,722.50$ \\
\hline Mauritius & 1 & $486,885.50$ & $209,370.00$ & $390,331.50$ & $13,047.50$ \\
\hline Mauritius & 4 & $351,304.50$ & $138,424.00$ & $258,993.50$ & $8,630.00$ \\
\hline Mauritius & 14 & $214,209.50$ & $88,073.00$ & $165,625.00$ & $5,463.00$ \\
\hline Mauritius & 49 & $422,540.50$ & $176,489.00$ & $329,162.50$ & $11,015.50$ \\
\hline Malawi & 1 & $6,206,020.50$ & $853,281.00$ & $1,590,558.50$ & $52,895.50$ \\
\hline Malawi & 4 & $4,609,105.50$ & $597,183.50$ & $1,114,078.50$ & $37,180.00$ \\
\hline Malawi & 14 & $2,414,871.50$ & $329,973.00$ & $616,215.00$ & $20,405.50$ \\
\hline Malawi & 49 & $5,394,534.00$ & $731,214.50$ & $1,362,680.00$ & $45,337.50$ \\
\hline Namibia & 1 & $805,968.50$ & $113,124.50$ & $211,467.00$ & $7,031.50$ \\
\hline Namibia & 4 & $594,974.50$ & $78,583.00$ & $146,712.50$ & $4,861.00$ \\
\hline Namibia & 14 & $271,679.50$ & $36,560.50$ & $68,778.50$ & $2,296.50$ \\
\hline Namibia & 49 & $696,764.50$ & $95,833.50$ & $179,065.50$ & $5,974.50$ \\
\hline Niger & 1 & $7,479,840.50$ & $911,995.00$ & $1,704,345.00$ & $56,835.50$ \\
\hline Niger & 4 & $5,361,960.00$ & $604,501.50$ & $1,131,297.50$ & $37,570.50$ \\
\hline Niger & 14 & $2,656,777.50$ & $323,438.50$ & $603,880.50$ & $20,051.50$ \\
\hline Niger & 49 & $6,407,713.00$ & $758,280.50$ & $1,417,828.00$ & $47,137.00$ \\
\hline Nigeria & 1 & $67,998,172.00$ & $10,931,386.50$ & $20,404,287.00$ & $679,437.50$ \\
\hline Nigeria & 4 & $51,622,542.50$ & $7,976,219.00$ & $14,878,213.00$ & $495,446.50$ \\
\hline Nigeria & 14 & $28,071,652.50$ & $4,656,256.00$ & $8,692,204.50$ & $289,283.50$ \\
\hline Nigeria & 49 & $59,789,002.00$ & $9,541,919.50$ & $17,807,958.50$ & $593,371.50$ \\
\hline Nicaragua & 1 & $2,235,573.00$ & $500,811.00$ & $937,662.50$ & $31,193.00$ \\
\hline Nicaragua & 4 & $1,671,927.50$ & $340,380.50$ & $632,680.50$ & $21,123.50$ \\
\hline Nicaragua & 14 & $917,568.00$ & $190,082.50$ & $355,496.00$ & $11,913.00$ \\
\hline Nicaragua & 49 & $1,954,965.00$ & $422,935.50$ & $790,201.00$ & $26,307.50$ \\
\hline Nepal & 1 & $9,915,594.50$ & $2,499,733.50$ & $4,665,603.00$ & $155,338.50$ \\
\hline Nepal & 4 & $7,426,283.00$ & $1,746,579.00$ & $3,269,303.00$ & $108,873.50$ \\
\hline Nepal & 14 & $3,596,042.00$ & $931,577.50$ & $1,736,856.00$ & $57,692.50$ \\
\hline Nepal & 49 & $8,680,618.50$ & $2,156,185.00$ & $4,028,305.50$ & $134,340.50$ \\
\hline Oman & 1 & $1,535,261.00$ & $176,798.50$ & $329,421.00$ & $10,993.00$ \\
\hline
\end{tabular}


medRxiv preprint doi: https://doi.org/10.1101/2020.08.23.20180299; this version posted August 25, 2020. The copyright holder for this preprint (which was not certified by peer review) is the author/funder, who has granted medRxiv a license to display the preprint in perpetuity.

It is made available under a CC-BY-NC-ND 4.0 International license.

\begin{tabular}{|c|c|c|c|c|c|}
\hline Oman & 4 & $1,072,384.00$ & $107,046.00$ & $199,923.00$ & $6,631.00$ \\
\hline Oman & 14 & $551,427.00$ & $50,614.00$ & $93,722.50$ & $3,132.50$ \\
\hline Oman & 49 & $1,289,638.50$ & $138,700.50$ & $258,894.00$ & $8,678.00$ \\
\hline Pakistan & 1 & $71,833,290.50$ & $13,106,615.00$ & $24,490,690.50$ & $816,437.00$ \\
\hline Pakistan & 4 & $53,017,172.50$ & $8,893,216.00$ & $16,571,105.50$ & $551,824.00$ \\
\hline Pakistan & 14 & $29,435,591.50$ & $5,229,562.50$ & $9,784,165.00$ & $324,959.50$ \\
\hline Pakistan & 49 & $62,541,662.00$ & $10,974,825.00$ & $20,499,024.50$ & $682,425.00$ \\
\hline Panama & 1 & $1,517,972.50$ & $471,387.00$ & $879,487.50$ & $29,338.50$ \\
\hline Panama & 4 & $1,105,635.50$ & $311,636.50$ & $581,917.50$ & $19,376.00$ \\
\hline Panama & 14 & $599,862.50$ & $178,411.50$ & $335,181.00$ & $11,120.50$ \\
\hline Panama & 49 & $1,313,913.50$ & $396,995.50$ & $740,170.50$ & $24,635.00$ \\
\hline Peru & 1 & $11,655,014.00$ & $3,620,110.00$ & $6,750,234.00$ & $224,803.00$ \\
\hline Peru & 4 & $8,460,494.50$ & $2,403,019.00$ & $4,479,917.00$ & $148,873.00$ \\
\hline Peru & 14 & $4,169,860.50$ & $1,246,737.00$ & $2,324,155.00$ & $77,271.50$ \\
\hline Peru & 49 & $10,081,293.50$ & $3,039,427.50$ & $5,677,001.50$ & $188,946.00$ \\
\hline Paraguay & 1 & $2,401,204.50$ & $602,571.50$ & $1,122,937.50$ & $37,455.50$ \\
\hline Paraguay & 4 & $1,771,743.00$ & $398,632.00$ & $744,466.50$ & $24,878.00$ \\
\hline Paraguay & 14 & $880,973.50$ & $208,728.50$ & $389,898.50$ & $12,896.50$ \\
\hline Paraguay & 49 & $2,085,255.00$ & $508,236.50$ & $946,491.00$ & $31,507.00$ \\
\hline West Bank and Gaza & 1 & $1,594,003.50$ & $226,570.50$ & $422,739.00$ & $14,105.00$ \\
\hline West Bank and Gaza & 4 & $1,189,222.50$ & $151,665.50$ & $281,435.00$ & $9,406.50$ \\
\hline West Bank and Gaza & 14 & $712,802.50$ & $95,452.50$ & $176,325.50$ & $5,948.50$ \\
\hline West Bank and Gaza & 49 & $1,387,924.50$ & $190,382.00$ & $355,232.00$ & $11,799.00$ \\
\hline Qatar & 1 & $899,032.50$ & $113,926.50$ & $212,464.50$ & $7,071.00$ \\
\hline Qatar & 4 & $641,023.00$ & $70,589.50$ & $132,073.50$ & $4,377.50$ \\
\hline Qatar & 14 & $266,723.50$ & $26,536.00$ & $49,248.00$ & $1,647.50$ \\
\hline Qatar & 49 & $766,587.00$ & $89,823.50$ & $168,594.50$ & $5,584.50$ \\
\hline Rwanda & 1 & $4,260,092.00$ & $653,278.50$ & $1,221,703.50$ & $40,608.00$ \\
\hline Rwanda & 4 & $3,212,134.50$ & $457,874.00$ & $855,829.50$ & $28,478.00$ \\
\hline Rwanda & 14 & $1,749,170.00$ & $255,902.00$ & $479,833.50$ & $16,021.50$ \\
\hline Rwanda & 49 & $3,746,403.00$ & $557,316.50$ & $1,041,050.00$ & $34,717.00$ \\
\hline Saudi Arabia & 1 & $11,941,083.50$ & $2,049,485.00$ & $3,823,720.50$ & $127,556.50$ \\
\hline Saudi Arabia & 4 & $8,911,360.50$ & $1,384,909.50$ & $2,587,572.50$ & $86,282.50$ \\
\hline Saudi Arabia & 14 & $5,395,467.50$ & $824,158.50$ & $1,538,146.50$ & $51,054.00$ \\
\hline Saudi Arabia & 49 & $10,445,557.00$ & $1,719,131.00$ & $3,203,677.50$ & $106,843.00$ \\
\hline Sudan & 1 & $14,419,983.00$ & $2,514,130.50$ & $4,680,808.50$ & $155,985.00$ \\
\hline Sudan & 4 & $10,877,482.50$ & $1,772,405.50$ & $3,314,864.00$ & $110,203.50$ \\
\hline Sudan & 14 & $5,777,423.00$ & $1,008,371.50$ & $1,878,300.50$ & $62,797.00$ \\
\hline
\end{tabular}


medRxiv preprint doi: https://doi.org/10.1101/2020.08.23.20180299; this version posted August 25, 2020. The copyright holder for this preprint (which was not certified by peer review) is the author/funder, who has granted medRxiv a license to display the preprint in perpetuity.

It is made available under a CC-BY-NC-ND 4.0 International license.

\begin{tabular}{|c|c|c|c|c|c|}
\hline Sudan & 49 & $12,629,479.00$ & $2,164,740.50$ & $4,038,214.50$ & $134,408.50$ \\
\hline Senegal & 1 & $5,506,929.50$ & $943,941.00$ & $1,765,632.50$ & $58,633.50$ \\
\hline Senegal & 4 & $4,152,655.50$ & $688,535.00$ & $1,284,545.00$ & $42,863.00$ \\
\hline Senegal & 14 & $2,285,377.00$ & $427,813.00$ & $800,324.00$ & $26,502.50$ \\
\hline Senegal & 49 & $4,809,000.50$ & $828,513.50$ & $1,548,749.50$ & $51,440.50$ \\
\hline Sierra Leone & 1 & $2,630,770.00$ & $424,739.50$ & $793,709.50$ & $26,428.50$ \\
\hline Sierra Leone & 4 & $1,994,333.00$ & $307,273.00$ & $574,350.00$ & $19,119.50$ \\
\hline Sierra Leone & 14 & $1,158,003.00$ & $191,212.00$ & $360,302.00$ & $12,060.50$ \\
\hline Sierra Leone & 49 & $2,319,759.00$ & $370,961.50$ & $690,722.50$ & $22,966.50$ \\
\hline El Salvador & 1 & $2,258,281.50$ & $726,963.50$ & $1,354,593.00$ & $45,160.50$ \\
\hline El Salvador & 4 & $1,664,921.50$ & $490,165.50$ & $916,503.50$ & $30,630.00$ \\
\hline El Salvador & 14 & $908,668.00$ & $289,893.50$ & $538,904.50$ & $17,905.50$ \\
\hline El Salvador & 49 & $1,965,853.00$ & $619,690.50$ & $1,156,953.50$ & $38,540.00$ \\
\hline Somalia & 1 & $5,054,908.00$ & $722,092.50$ & $1,346,267.50$ & $44,904.50$ \\
\hline Somalia & 4 & $3,796,053.50$ & $508,883.00$ & $947,770.50$ & $31,691.00$ \\
\hline Somalia & 14 & $1,969,685.00$ & $281,476.00$ & $522,971.00$ & $17,450.00$ \\
\hline Somalia & 49 & $4,408,720.50$ & $619,059.00$ & $1,154,447.00$ & $38,523.50$ \\
\hline South Sudan & 1 & $3,696,526.00$ & $575,956.50$ & $1,078,140.00$ & $35,884.00$ \\
\hline South Sudan & 4 & $2,762,595.00$ & $406,328.00$ & $758,511.00$ & $25,346.00$ \\
\hline South Sudan & 14 & $1,282,595.00$ & $203,062.00$ & $381,821.00$ & $12,566.00$ \\
\hline South Sudan & 49 & $3,220,523.50$ & $493,716.50$ & $922,740.50$ & $30,649.50$ \\
\hline Sao Tome and Principe & 1 & $72,283.50$ & $11,231.50$ & $20,945.00$ & 696.00 \\
\hline Sao Tome and Principe & 4 & $54,913.00$ & $8,013.50$ & $15,109.50$ & 500.00 \\
\hline Sao Tome and Principe & 14 & $29,481.50$ & $4,448.00$ & $8,484.00$ & 283.50 \\
\hline Sao Tome and Principe & 49 & $63,572.00$ & $9,662.00$ & $17,970.50$ & 606.50 \\
\hline Eswatini & 1 & $363,364.00$ & $62,143.50$ & $115,928.00$ & $3,869.00$ \\
\hline Eswatini & 4 & $266,346.00$ & $40,923.50$ & $76,890.50$ & $2,558.00$ \\
\hline Eswatini & 14 & $119,567.50$ & $18,886.00$ & $35,996.50$ & $1,187.00$ \\
\hline Eswatini & 49 & $313,533.00$ & $51,987.50$ & $96,273.50$ & $3,204.00$ \\
\hline Seychelles & 1 & $35,263.00$ & $12,022.50$ & $22,569.50$ & 746.00 \\
\hline Seychelles & 4 & $25,064.00$ & $7,926.50$ & $14,905.00$ & 495.50 \\
\hline Seychelles & 14 & $14,434.00$ & $4,800.50$ & $8,982.50$ & 294.50 \\
\hline Seychelles & 49 & $30,440.50$ & $10,024.50$ & $18,709.50$ & 625.00 \\
\hline Syrian Arab Republic & 1 & $5,792,480.00$ & $989,564.50$ & $1,847,430.00$ & $61,580.00$ \\
\hline Syrian Arab Republic & 4 & $4,370,151.50$ & $718,417.00$ & $1,339,837.50$ & $44,443.00$ \\
\hline Syrian Arab Republic & 14 & $2,590,708.00$ & $461,948.50$ & $861,860.00$ & $28,637.00$ \\
\hline Syrian Arab Republic & 49 & $5,101,506.50$ & $858,728.00$ & $1,601,689.50$ & $53,348.50$ \\
\hline Chad & 1 & $5,065,942.50$ & $601,091.00$ & $1,124,428.00$ & $37,284.50$ \\
\hline
\end{tabular}


medRxiv preprint doi: https://doi.org/10.1101/2020.08.23.20180299; this version posted August 25, 2020. The copyright holder for this preprint (which was not certified by peer review) is the author/funder, who has granted medRxiv a license to display the preprint in perpetuity.

It is made available under a CC-BY-NC-ND 4.0 International license.

\begin{tabular}{|c|c|c|c|c|c|}
\hline Chad & 4 & $3,620,549.50$ & $399,236.00$ & $745,414.50$ & $24,835.00$ \\
\hline Chad & 14 & $1,795,297.50$ & $213,052.50$ & $398,405.50$ & $13,124.00$ \\
\hline Chad & 49 & $4,330,613.50$ & $500,212.50$ & $933,773.00$ & $31,055.00$ \\
\hline Togo & 1 & $2,738,321.50$ & $409,696.00$ & $766,658.50$ & $25,515.50$ \\
\hline Togo & 4 & $2,084,481.50$ & $294,394.50$ & $549,644.00$ & $18,293.50$ \\
\hline Togo & 14 & $1,117,765.50$ & $165,863.50$ & $310,016.00$ & $10,260.50$ \\
\hline Togo & 49 & $2,416,837.50$ & $353,371.50$ & $660,994.00$ & $21,999.00$ \\
\hline Tajikistan & 1 & $2,980,380.50$ & $506,624.00$ & $946,025.50$ & $31,461.00$ \\
\hline Tajikistan & 4 & $2,132,996.50$ & $334,988.50$ & $624,270.50$ & $20,882.50$ \\
\hline Tajikistan & 14 & $1,073,616.50$ & $173,478.00$ & $325,632.50$ & $10,814.50$ \\
\hline Tajikistan & 49 & $2,558,536.50$ & $423,792.00$ & $791,527.50$ & $26,444.00$ \\
\hline Tunisia & 1 & $4,156,065.00$ & $1,421,020.00$ & $2,652,235.00$ & $88,351.50$ \\
\hline Tunisia & 4 & $2,993,242.50$ & $934,481.50$ & $1,744,116.00$ & $58,013.50$ \\
\hline Tunisia & 14 & $1,711,642.00$ & $556,560.50$ & $1,039,231.00$ & $34,717.50$ \\
\hline Tunisia & 49 & $3,578,598.00$ & $1,197,555.50$ & $2,241,228.00$ & $74,470.50$ \\
\hline Turkey & 1 & $31,029,721.00$ & $11,408,352.00$ & $21,265,807.50$ & $708,020.00$ \\
\hline Turkey & 4 & $23,141,897.50$ & $7,967,971.50$ & $14,847,503.50$ & $495,399.50$ \\
\hline Turkey & 14 & $14,342,055.00$ & $5,342,495.00$ & $9,982,584.00$ & $331,482.50$ \\
\hline Turkey & 49 & $27,262,980.00$ & $9,879,607.50$ & $18,427,321.50$ & $614,391.00$ \\
\hline Tanzania & 1 & $19,439,044.50$ & $2,891,250.00$ & $5,398,275.50$ & $180,022.50$ \\
\hline Tanzania & 4 & $14,683,606.50$ & $2,076,715.50$ & $3,872,581.00$ & $129,155.00$ \\
\hline Tanzania & 14 & $7,943,080.00$ & $1,196,374.50$ & $2,231,611.50$ & $74,239.50$ \\
\hline Tanzania & 49 & $17,106,351.50$ & $2,514,752.00$ & $4,689,457.00$ & $156,667.50$ \\
\hline Uganda & 1 & $14,035,584.50$ & $2,241,331.50$ & $4,179,978.50$ & $139,292.00$ \\
\hline Uganda & 4 & $10,795,699.00$ & $1,767,713.00$ & $3,302,956.50$ & $109,887.00$ \\
\hline Uganda & 14 & $9,634,472.00$ & $1,547,767.00$ & $2,892,963.50$ & $96,319.50$ \\
\hline Uganda & 49 & $12,625,196.50$ & $2,044,288.00$ & $3,816,799.50$ & $126,941.00$ \\
\hline Uruguay & 1 & $1,326,201.50$ & $679,333.50$ & $1,267,820.00$ & $42,256.00$ \\
\hline Uruguay & 4 & $938,231.00$ & $445,262.00$ & $828,683.00$ & $27,689.50$ \\
\hline Uruguay & 14 & $516,148.00$ & $261,647.50$ & $487,979.00$ & $16,297.00$ \\
\hline Uruguay & 49 & $1,141,786.50$ & $570,625.00$ & $1,062,322.00$ & $35,429.00$ \\
\hline Venezuela, RB & 1 & $10,135,488.00$ & $3,272,693.50$ & $6,112,276.00$ & $203,631.50$ \\
\hline Venezuela, RB & 4 & $7,385,224.00$ & $2,226,216.00$ & $4,152,468.50$ & $138,283.50$ \\
\hline Venezuela, RB & 14 & $4,478,616.50$ & $1,444,018.50$ & $2,687,084.50$ & $89,718.00$ \\
\hline Venezuela, RB & 49 & $8,782,756.00$ & $2,799,109.50$ & $5,232,723.50$ & $173,968.50$ \\
\hline Yemen, Rep. & 1 & $8,978,939.50$ & $1,189,341.00$ & $2,223,058.50$ & $74,107.50$ \\
\hline Yemen, Rep. & 4 & $6,443,445.50$ & $752,402.00$ & $1,407,658.50$ & $46,808.50$ \\
\hline Yemen, Rep. & 14 & $3,134,621.00$ & $389,461.00$ & $723,166.50$ & $23,984.50$ \\
\hline
\end{tabular}


medRxiv preprint doi: https://doi.org/10.1101/2020.08.23.20180299; this version posted August 25, 2020. The copyright holder for this preprint (which was not certified by peer review) is the author/funder, who has granted medRxiv a license to display the preprint in perpetuity.

It is made available under a CC-BY-NC-ND 4.0 International license.

\begin{tabular}{|l|r|r|r|r|r|} 
Yemen, Rep. & 49 & $7,619,708.50$ & $969,122.50$ & $1,816,360.50$ & $60,463.00$ \\
\hline South Africa & 1 & $20,484,691.50$ & $4,996,026.50$ & $9,320,556.00$ & $310,571.50$ \\
\hline South Africa & 4 & $15,232,036.50$ & $3,438,007.00$ & $6,410,413.00$ & $213,792.00$ \\
\hline South Africa & 14 & $8,795,217.00$ & $2,079,588.50$ & $3,890,084.50$ & $129,416.00$ \\
\hline South Africa & 49 & $17,929,452.50$ & $4,284,328.50$ & $7,988,411.00$ & $266,130.00$ \\
\hline Zambia & 1 & $5,908,589.50$ & $719,278.00$ & $1,341,163.00$ & $44,602.50$ \\
\hline Zambia & 4 & $4,464,221.00$ & $514,499.50$ & $966,758.00$ & $32,042.50$ \\
\hline Zambia & 14 & $2,130,687.00$ & $259,718.00$ & $486,323.50$ & $16,191.50$ \\
\hline Zambia & 49 & $5,177,648.00$ & $620,034.50$ & $1,154,007.00$ & $38,561.00$ \\
\hline Zimbabwe & 1 & $4,572,303.50$ & $991,498.50$ & $1,852,341.50$ & $61,683.50$ \\
\hline Zimbabwe & 4 & $3,484,742.00$ & $817,788.00$ & $1,524,034.50$ & $50,874.50$ \\
\hline Zimbabwe & 14 & $3,275,359.00$ & $779,772.50$ & $1,450,085.50$ & $48,299.00$ \\
\hline Zimbabwe & 49 & $4,110,615.00$ & $926,563.00$ & $1,728,563.00$ & $57,739.50$ \\
\hline
\end{tabular}


medRxiv preprint doi: https://doi.org/10.1101/2020.08.23.20180299; this version posted August 25, 2020. The copyright holder for this preprint (which was not certified by peer review) is the author/funder, who has granted medRxiv a license to display the preprint in perpetuity.

It is made available under a CC-BY-NC-ND 4.0 International license .

Supplemental S2. Parameters and process used in health resource and costing model

\section{S2.1. Summary}

We summarise here the main parameters used in the estimates of health resources and costing. Further details and references are then provided in the following sections.

In summary, there are four steps in our calculations:

1. Calculation of unit costs per activity for three base countries: Ethiopia (low-income country or 'LIC'), Pakistan (lower-middle income country or 'LMIC') and South Africa (upper-middle income country or 'UMIC')

2. Extrapolation of unit costs in base countries to calculate unit costs across 84 LICS, LMICs and UMICS

3. Calculation of total costs per country using country-specific unit costs, modelled data on the number of cases, hospitalisations and deaths, as well as other epidemiological and economic assumptions

4. Calculation of country-specific per capita costs, as well as per capita costs as a proportion of gross domestic product (GDP) per capita and various measures of health expenditure per capita

S2.2 Calculation of unit costs per activity for three base countries

\section{S2.2.1 General Approach}

A full economic costing was carried out over a one-year time horizon. Costs were constructed using a bottom-up ingredients-based technique. The costing was carried out from a health systems perspective and included both direct (e.g. medicines) and indirect costs (e.g. facility overheads). No above-service delivery costs were included.

The eighty-four countries chosen met three inclusion criteria: 1) be classified as low-income, lowermiddle income or upper-middle income by the World Bank [17], 2) be included in the list of 92 countries for which epidemiological modelling data was available from Pearson et al (2020) [16], and 3 ) have recent available GDP per capita data in order to carry out cost extrapolation between countries [17].

\section{S2.2.2 Intervention costs}

We used official WHO guidance to identify areas related to critical preparedness, readiness and response actions for COVID-19 to define a set of interventions involved in a national response to the pandemic. This guidance identifies 6 priority areas of work and is further sub-divided into 13 activities. 
- Emergency response mechanisms at the national level

- Risk communication and community engagement

- Case finding, contact tracing and management

- Surveillance

- Public health measures

- Case management

For the first five areas of work we considered only WHO guidance to define the resource use. For case management costs we assumed less resource-intensive activities that we felt were more plausible in low- and middle-income settings ('real-world'). Assumptions on 'real world' resource use were based on the clinical expertise of members of the research team and are detailed below.

Following this guidance, we generated a list of unit costs to be brought together with the COVID epidemiological model to estimate resource needs, as detailed in Table S2.2.

Table S2.2.2.1 Intervention description and unit costs

\begin{tabular}{|l|l|}
\hline Activity & Activity unit costs \\
& \\
\hline 1.a. Emergency Response Mechanisms: National level & Per country per day \\
\hline 1.b. Emergency Response Mechanisms: Training of health staff & One-off per site \\
\hline 2. Risk communication \& community engagement & Per country per day \\
\hline 3.a. Case finding, contact tracing and management: Contact tracing & Per person contacted \\
\hline $\begin{array}{l}\text { 3.b. Case finding, contact tracing and management: Quarantine of } \\
\text { contacts }\end{array}$ & Per person quarantined \\
\hline 4.a. Surveillance: Case notification & Per positive case \\
\hline 4.b. Surveillance: Reporting (national level) & Per country per week \\
\hline 5. Public health measures: Hygiene education & Per education campaign per month \\
\hline 6. Screening and diagnosis & Per person screened and tested \\
\hline 7.a. Case Management: Home-based care & Per person requiring home-based care \\
\hline 7.b. Case Management: Hospital-based (severe case) & Per day of hospitalisation (severe case) \\
\hline 7.c. Case Management: Hospital-based (critical case) & Per day of hospitalisation (critical case) \\
\hline 7.d. Case Management: Death & Per COVID-related death \\
\hline
\end{tabular}

\section{S.2.2.3. Defining Inputs, Costs per Input and Quantities of Inputs}

For each of the abovementioned activities we used an ingredients based costing to identify a series of inputs (e.g. junior-level government worker day). For each input we estimated a number of units (e.g. three days of work) and a country-specific price. The costs of each input were identified using a 
medRxiv preprint doi: https://doi.org/10.1101/2020.08.23.20180299; this version posted August 25, 2020. The copyright holder for this preprint (which was not certified by peer review) is the author/funder, who has granted medRxiv a license to display the preprint in perpetuity.

It is made available under a CC-BY-NC-ND 4.0 International license.

range of sources, according to availability of recent primary cost data and appropriateness of cost estimates to the COVID pattern of care. See the table below.

Table S2.2.3.1 Quantities and Unit Costs per Input per Activity per Country

\begin{tabular}{|c|c|c|c|c|c|c|c|}
\hline \multirow[t]{2}{*}{ Component } & \multirow{2}{*}{$\begin{array}{l}\text { Number of } \\
\text { Units per } \\
\text { Input }\end{array}$} & \multicolumn{6}{|c|}{ Unit cost per Inputs } \\
\hline & & & Ethiopia & & Pakistan & & South Africa \\
\hline \multicolumn{8}{|c|}{ 1.a. Emergency Response Mechanisms: National level } \\
\hline Working day (junior level govt) & 10 & $\$$ & 12.27 & $\$$ & 13.07 & $\$$ & 194.66 \\
\hline Working day (senior level govt) & 10 & $\$$ & 17.29 & $\$$ & 23.94 & $\$$ & 256.72 \\
\hline Meeting/training costs per person per day & 20 & $\$$ & 13.18 & $\$$ & 20.44 & $\$$ & 159.17 \\
\hline Total: & & $\$$ & 559.26 & $\$$ & 778.90 & $\$$ & $7,697.16$ \\
\hline \multicolumn{8}{|c|}{ 1.b. Emergency Response Mechanisms: Training of health staff } \\
\hline Working day (health care workers) & 250 & $\$$ & 4.93 & $\$$ & 10.43 & $\$$ & 97.58 \\
\hline Working day (junior level govt) & 10 & $\$$ & 12.27 & $\$$ & 13.07 & $\$$ & 194.66 \\
\hline Working day (senior level govt) & 1 & $\$$ & 17.29 & $\$$ & 23.94 & $\$$ & 256.72 \\
\hline Meeting/ training costs per person per $d a y$ & 261 & $\$$ & 13.18 & $\$$ & 20.44 & $\$$ & 159.17 \\
\hline Total: & & $\$$ & $4,813.58$ & $\$$ & $8,096.53$ & $\$$ & $68,141.36$ \\
\hline \multicolumn{8}{|l|}{ 2. Risk communication \& community en gagement } \\
\hline Working day (junior level govt) & 3 & $\$$ & 12.27 & $\$$ & 13.07 & $\$$ & 194.66 \\
\hline Working day (senior level govt) & 2 & $\$$ & 17.29 & $\$$ & 23.94 & $\$$ & 256.72 \\
\hline Media costs per day & 1 & $\$$ & 2.74 & $\$$ & 4.58 & $\$$ & 36.00 \\
\hline Total: & & $\$$ & 74.14 & $\$$ & 91.67 & $\$$ & $1,133.44$ \\
\hline \multicolumn{8}{|c|}{ 3.a. Case find ing, contact tracing and management: Contact tracing } \\
\hline Working day (junior level govt) & 0.1 & $\$$ & 12.27 & $\$$ & 13.07 & $\$$ & 194.66 \\
\hline Cont act tracing household visit & 0.33 & $\$$ & 2.08 & $\$$ & 3.02 & $\$$ & 13.68 \\
\hline Cont act tracing phone call & 0.67 & $\$$ & 2.34 & $\$$ & 0.34 & $\$$ & 3.31 \\
\hline Total: & & $\$$ & 3.48 & $\$$ & 2.54 & $\$$ & 26.23 \\
\hline \multicolumn{8}{|c|}{ 3.b. Case finding, contact tracing and management: Quarantine of contacts } \\
\hline Working day (health care workers) & 0.1 & $\$$ & 4.93 & $\$$ & 10.43 & $\$$ & 97.58 \\
\hline Working day (junior level govt) & 0.1 & $\$$ & 12.27 & $\$$ & 13.07 & $\$$ & 194.66 \\
\hline Total: & & $\$$ & 1.72 & $\$$ & 2.35 & $\$$ & 29.22 \\
\hline \multicolumn{8}{|l|}{ 4.a. Surveillance: Case notification } \\
\hline Working day (health care workers) & 0.1 & $\$$ & 4.93 & $\$$ & 10.43 & $\$$ & 97.58 \\
\hline Working day (junior level govt) & 0.1 & $\$$ & 12.27 & $\$$ & 13.07 & $\$$ & 194.66 \\
\hline Total: & & $\$$ & 1.72 & $\$$ & 2.35 & $\$$ & 29.22 \\
\hline \multicolumn{8}{|l|}{ 4.b. Surveillance: Reporting (national level) } \\
\hline Working day (health care workers) & 0.5 & $\$$ & 4.93 & $\$$ & 10.43 & $\$$ & 97.58 \\
\hline Working day (junior level govt) & 0.1 & $\$$ & 12.27 & $\$$ & 13.07 & $\$$ & 194.66 \\
\hline Total: & & $\$$ & 3.69 & $\$$ & 6.52 & $\$$ & 68.26 \\
\hline \multicolumn{8}{|l|}{ 5. Public health measures: Hygiene education } \\
\hline Working day (junior level govt) & 2 & $\$$ & 12.27 & $\$$ & 10.43 & $\$$ & 97.58 \\
\hline Working day (senior level govt) & 1 & $\$$ & 17.29 & $\$$ & 13.07 & $\$$ & 194.66 \\
\hline Media costs per day & 1 & $\$$ & 2.74 & $\$$ & 4.58 & $\$$ & 36.00 \\
\hline Total: & & $\$$ & 44.58 & $\$$ & 38.51 & $\$$ & 425.83 \\
\hline \multicolumn{8}{|l|}{ 6. Screening and diagn osis } \\
\hline Ambulancetrip & 0.0001 & $\$$ & 4.80 & $\$$ & 9.51 & $\$$ & 60.41 \\
\hline Isolation pod/ diagnostic visit & 2 & $\$$ & 0.49 & $\$$ & 0.49 & $\$$ & 7.97 \\
\hline Outp atient visit oral history & 1 & $\$$ & 3.57 & $\$$ & 0.47 & $\$$ & 8.02 \\
\hline Outp atient visit physical exam & 1 & $\$$ & 3.57 & $\$$ & 0.47 & $\$$ & 8.02 \\
\hline
\end{tabular}


medRxiv preprint doi: https://doi.org/10.1101/2020.08.23.20180299; this version posted August 25, 2020. The copyright holder for this preprint (which was not certified by peer review) is the author/funder, who has granted medRxiv a license to display the preprint in perpetuity.

It is made available under a CC-BY-NC-ND 4.0 International license.

\begin{tabular}{|c|c|c|c|c|c|c|c|}
\hline Outp atient visit specimen collect ion & 1 & $\$$ & 4.88 & $\$$ & 1.09 & $\$$ & 17.15 \\
\hline CoVD19 test (PCR) & 1 & $\$$ & 23.98 & $\$$ & 23.98 & $\$$ & 23.98 \\
\hline Total: & & $\$$ & 36.97 & $\$$ & 26.98 & $\$$ & 73.12 \\
\hline \multicolumn{8}{|l|}{ 7.a. Case Management: Home-based care } \\
\hline Home-based care bed-day & 5 & $\$$ & 0.94 & $\$$ & 0.61 & $\$$ & 11.65 \\
\hline Community-based care via GP & 2 & $\$$ & 9.11 & $\$$ & 4.71 & $\$$ & 44.16 \\
\hline Total: & & $\$$ & 22.90 & $\$$ & 12.45 & $\$$ & 146.57 \\
\hline \multicolumn{8}{|c|}{ 7.b. Case Management: Hospittal-based (severe case) } \\
\hline Inpatient ward bed-day (severe) & 1 & $\$$ & 29.90 & $\$$ & 31.54 & $\$$ & 96.66 \\
\hline \multicolumn{8}{|l|}{ Diagnostics } \\
\hline Pulse oximetry & 0.125 & $\$$ & 0.00 & $\$$ & 0.00 & $\$$ & 0.00 \\
\hline Chest $\mathrm{X}$-ray & 0.125 & $\$$ & 27.35 & $\$$ & 2.79 & $\$$ & 21.86 \\
\hline Full blood count & 0.125 & $\$$ & 2.37 & $\$$ & 2.29 & $\$$ & 24.28 \\
\hline Blood urea and electrolyte test & 0.125 & $\$$ & 4.20 & $\$$ & 2.53 & $\$$ & 2.87 \\
\hline c-reactive protein test & 0.125 & $\$$ & 2.34 & $\$$ & 0.32 & $\$$ & 5.15 \\
\hline HIVt est & 0.125 & $\$$ & 4.38 & $\$$ & 3.87 & $\$$ & 17.13 \\
\hline CoVD19 test (PCR) & 0 & $\$$ & 23.98 & $\$$ & 23.98 & $\$$ & 23.98 \\
\hline Mala ria test & 0.125 & $\$$ & 0.19 & $\$$ & 0.19 & $\$$ & 0.19 \\
\hline Haemoglobin test & 0.125 & $\$$ & 2.29 & $\$$ & 2.29 & $\$$ & 2.29 \\
\hline Total: & & $\$$ & 35.29 & $\$$ & 33.32 & $\$$ & 105.88 \\
\hline \multicolumn{8}{|c|}{ 7.c. Case Management: Hospital-based (critical case) } \\
\hline Inpatient ward bed-day (critical) & 0.33 & 30.65 & & 32.2 & & 97.4 & \\
\hline ITU bed-day & 0.67 & $\$$ & 104.48 & $\$$ & 101.99 & $\$$ & 662.71 \\
\hline \multicolumn{8}{|c|}{ Additional resourcing per COVID-related complication } \\
\hline Acut e respiratory distress syndrome (ARDS) & 0.47 & $\$$ & 22.46 & $\$$ & 22.46 & $\$$ & 22.46 \\
\hline Acut e kidney injury days & 0.04 & $\$$ & 10.60 & $\$$ & 10.60 & $\$$ & 10.60 \\
\hline Acut e cardiac injury days & 0.06 & $\$$ & 46.25 & $\$$ & 46.25 & $\$$ & 46.25 \\
\hline Liver dysfunction days & 0.06 & $\$$ & 89.32 & $\$$ & 89.32 & $\$$ & 89.32 \\
\hline Pneumothorax days & 0.01 & $\$$ & 6.66 & $\$$ & 6.77 & $\$$ & 7.02 \\
\hline Hospital-acquired pneumonia days & 0.05 & $\$$ & 18.85 & $\$$ & 18.85 & $\$$ & 18.85 \\
\hline Bacteraemia days & 0.01 & $\$$ & 32.55 & $\$$ & 32.55 & $\$$ & 32.55 \\
\hline Urinary tract infection days & 0.01 & $\$$ & 9.03 & $\$$ & 9.03 & $\$$ & 9.03 \\
\hline Septic shock days & 0.05 & $\$$ & 0.64 & $\$$ & 0.67 & $\$$ & 0.75 \\
\hline \multicolumn{8}{|l|}{ Diagnostics } \\
\hline Pulse oximetry & 10 & $\$$ & 0.00 & $\$$ & 0.00 & $\$$ & 0.00 \\
\hline Chest X-ray & 10 & $\$$ & 27.35 & $\$$ & 2.79 & $\$$ & 21.86 \\
\hline Full blood count & 10 & $\$$ & 2.37 & $\$$ & 2.29 & $\$$ & 24.28 \\
\hline Blood urea and electrolyte test & 10 & $\$$ & 4.20 & $\$$ & 2.53 & $\$$ & 2.87 \\
\hline C-reactive protein test & 10 & $\$$ & 2.34 & $\$$ & 0.32 & $\$$ & 5.15 \\
\hline Venous blood gas test & 10 & $\$$ & 4.23 & $\$$ & 4.23 & $\$$ & 4.23 \\
\hline HIVtest & 0.1 & $\$$ & 4.38 & $\$$ & 3.87 & $\$$ & 17.13 \\
\hline COVD19 test (PCR) & 0 & $\$$ & 23.98 & $\$$ & 23.98 & $\$$ & 23.98 \\
\hline Mala ria test & 0.1 & $\$$ & 0.19 & $\$$ & 0.19 & $\$$ & 0.19 \\
\hline Haemoglobin test & 0.1 & $\$$ & 2.29 & $\$$ & 2.29 & $\$$ & 2.29 \\
\hline Total: & & $\$$ & 505.56 & $\$$ & 221.18 & $\$$ & $1,081.94$ \\
\hline \multicolumn{8}{|l|}{ 7.d. Case Management: Death } \\
\hline Body Bag & 1 & $\$$ & 64.52 & $\$$ & 64.52 & $\$$ & 64.52 \\
\hline Total: & & $\$$ & 64.52 & $\$$ & 64.52 & $\$$ & 64.52 \\
\hline
\end{tabular}




\section{S.2.2.4 Quantities}

Planning and Management Activities (Activities 1-5)

Quantities of working days required for planning and management activities were estimated from expert consultation as part of the Diseases Control Priorities project. For other activities, quantities were estimated based on requirements for similar activities for tuberculosis (TB) such as contact tracing from the VALUE TB study and previous studies in South Africa (more below).

\section{Diagnosis and Clinical Management (Activities 6-7)}

The number of days per patient in general ward and in ICU was set at 8 and 10 respectively, and was set to match the assumptions in the epidemiological model $[16,19,20]$. Following expert clinician advise we assumed that one-third of critical patient bed days would be treated the general ward and two-thirds in the ICU.

The likelihood of additional COVID-related complications (per day) were estimated using evidence on the clinical course of COVID from patients in Wuhan, China ${ }^{17}$, as were assumptions on the duration of symptoms ${ }^{18}$ [21]. The number of diagnostic tests per hospitalisation was carried out in consultation with expert clinicians in essential critical care.

\section{S.2.2.5 Input unit costs}

\section{S2.2.5.1 Estimation of non-bed-day costs (Pakistan)}

An ingredients-based approach was used to calculate most of the service costs and prices for Pakistan. The data used was collected as part of the Disease Control Priorities 3-Universal Health Coverage (DCP3-UHC) project. For other countries primary data from the TB studies was used (see below).

For Pakistan, staff-related costs were constructed using federal-level pay scales. For most outputs, the number of minutes of staff required per activity were estimated via expert opinion obtained from clinicians working in the Health Planning, System Strengthening \& Information Analysis Unit (HPSIU) in the Ministry of National Health Services Regulations and Coordination of Pakistan. For outputs where this was unavailable, health economists agreed a plausible assumed value.

Drug regimens were costed using resource use data obtained through expert opinion (HPSIU) and a number of price sources. An assessment of strengths and weaknesses of different price sources was conducted and hierarchy of sources was established. The primary source of price data was the Sindh Health Department Procurement Price list. If a price was unavailable, the Federal Wholesale Price List for Generic Medicines was used as a second option. As a last resort, private sector market prices were used. 
Cost on supplies and equipment were similarly constructed. Resource use was determined through expert opinion (HPSIU) and price source hierarchy established. The primary source was the Medical Emergency Resilience Fund 2019-2020, and a secondary source was private sector market prices.

For all countries, for additional diagnostic and radiology costs (beyond those available from the TB data) were estimated using available literature and market prices. We assessed strengths and weaknesses of different price sources. For example, we used the 'Costing and Pricing of Services in Private Hospitals of Lahore: Summary Report' as our primary source as it contained a methodological appendix that suggested that an ingredients-based approach consistent with ours was followed. If some prices were unavailable we used user fees from the Pakistan Institute of Medical Sciences, procurement prices from the Medical Emergency Resilience Fund procurement prices and user fees from the Aga Khan University Hospital. Space costs were estimated using data from budget documents from the Federal government (Islamabad Capital Territory Health Infrastructure PC-1).

Oxygen therapy costs per bed-day were calculated by estimating the number of cylinders consumed in 24 hours at different flow rates, assumed to be $10 \mathrm{~L}$ per minute in the general ward and $30 \mathrm{~L} \mathrm{per}$ minute in the ICU. Cylinder duration (hours) was estimated by dividing pressure by the number of litres per minute, assuming a standard cylinder size of $4.6 \mathrm{~kg}$, filled at 1,900 psi pressure [22]. Cost per cylinder was obtained from the South African online catalogue of a manufacturer that is active in both South Africa and Pakistan [23].

\section{S2.2.5.2 Estimation of non-bed-day costs (Ethiopia and South Africa)}

For Ethiopia the main source of cost data was the VALUE TB study. Cost data collected from a health provider perspective to estimate the economic costs of TB-related health services. Full costs of health services were estimated, reflecting their implementation in the 'real world' rather than using a 'per protocol' costing approach. Cost data collection was retrospective, over a one-year period to minimize the risk of bias due to seasonality. Resource use was measured in the VALUE TB study using both top-down and bottom-up methods wherever possible, to allow for comparison. The costs included in the current cost model reflected an average of top-down and bottom-up costs by site.

For South Africa, we used primary data from the XTEND trial (nurses and lay health workers) ${ }^{19}$. This was updated using more recent data on contact tracing and update prices collected on an on-going trial of TB preventative therapy (WHIP $\mathrm{WB}_{3}$ ) (ClinicalTrials.gov, 2020).

Some of the COVID-19 interventions were outside the scope of the VALUE TB, XTEND, and WHIP ${ }_{3} T B$ studies. Values for which a primary unit cost was partially or entirely unavailable from Value TB are listed in the Table below. For these interventions, resource use from Pakistan (DCP) was then used with local prices (see list below).

Table S.2.2.5.3.1Inputs for which unit costs were estimated for South Africa and Ethiopia using resource use from $D C P$

\begin{tabular}{|l|}
\hline Planning \& coordination activities \\
\hline Working day (mid-level facility) \\
\hline Working day (junior level govt) \\
\hline Working day (senior level govt) \\
\hline Meeting/training costs per day \\
\hline Media costs per day \\
\hline
\end{tabular}


medRxiv preprint doi: https://doi.org/10.1101/2020.08.23.20180299; this version posted August 25, 2020. The copyright holder for this preprint (which was not certified by peer review) is the author/funder, who has granted medRxiv a license to display the preprint in perpetuity.

It is made available under a CC-BY-NC-ND 4.0 International license .

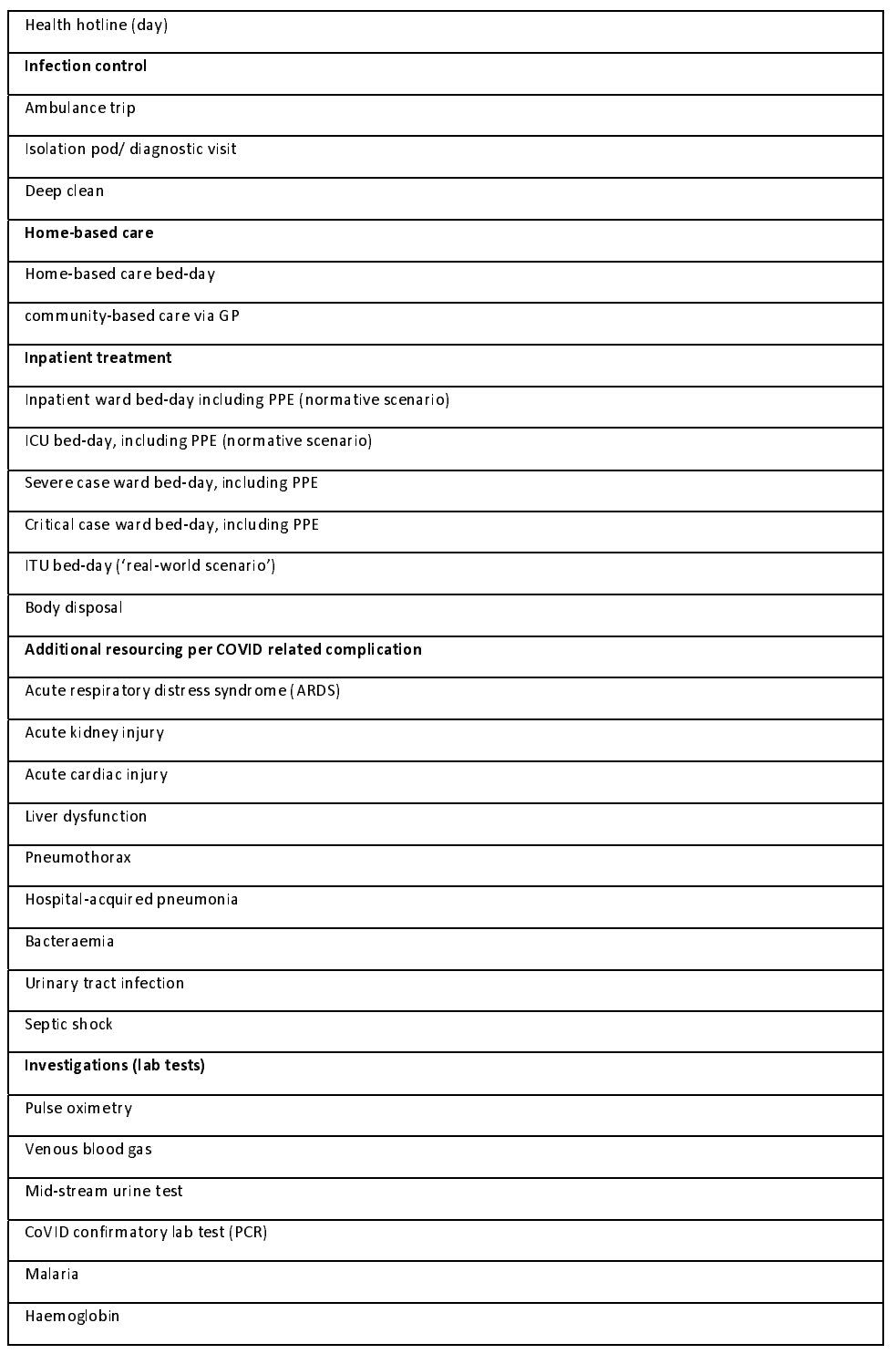

Where costs were applied from Pakistan health care inputs were classified as either tradeable, as in the case of medical equipment and supplies for diagnostic tests and procedures, and non-tradeable, including non-medical supplies, capital and overhead costs. For tradable inputs, where countryspecific price estimates were not available from primary data or from the published literature, the estimate from Pakistan was applied to other countries. For non-tradable inputs, the estimate from Pakistan was adjusted by an amount reflecting the difference in the two countries' GDP (PPP). The rationale behind this approach is that, while exchange rate may be influenced by government policy, PPP seeks to equalise the purchasing power of different currencies and, as such, may better reflect differentials in salaries and other non-tradable prices across countries. 
medRxiv preprint doi: https://doi.org/10.1101/2020.08.23.20180299; this version posted August 25, 2020. The copyright holder for this preprint (which was not certified by peer review) is the author/funder, who has granted medRxiv a license to display the preprint in perpetuity.

It is made available under a CC-BY-NC-ND 4.0 International license .

Table S2.2.5.4 Relative GDP adjustment factors

\begin{tabular}{|c|c|c|c|c|c|c|c|}
\hline & & & Relative GDP & PPP) & & & \\
\hline & $\begin{array}{l}\text { Exchange } \\
\text { rate (US\$) }\end{array}$ & $\begin{array}{l}\text { GDP per } \\
\text { capita by } \\
\text { country (US\$ } \\
\text { PPP) }\end{array}$ & Pakistan & $\begin{array}{l}\text { South } \\
\text { Africa }\end{array}$ & Kenya & India & Ethiopia \\
\hline Pakistan & 155.00 & $5,567.06$ & 1.00 & 0.41 & 0.87 & 0.72 & 2.75 \\
\hline South Africa & 32.26 & $2,022.14$ & 2.46 & 1.00 & 3.73 & 1.76 & 6.77 \\
\hline Kenya & 76.92 & $7,762.88$ & 0.62 & 0.25 & 1.00 & 0.45 & 1.71 \\
\hline India & 104.17 & $3,467.56$ & 1.39 & 0.57 & 1.18 & 1.00 & 3.84 \\
\hline Ethiopia & 16.95 & $13,686.88$ & 0.36 & 0.15 & 0.45 & 0.26 & 1.00 \\
\hline
\end{tabular}

\section{S2.2.5.5 Estimation of bed-day costs (all countries)}

We took an ingredients approach to estimating the costs of general ward and ICU ward bed days, as these were major cost drivers in our cost model. We estimated the plausible number of nursing hours per bed day in an LMIC setting through consultation with members of the research team who have expertise in critical care in LMICs. In ICU the assumption of nurse to patient ratio would be 1:1; in the general ward the ratio would be 1:6 during the day time and 1:20 in the night.

To understand the full range of inputs required we obtained the underlying costing data set provided by the authors of a recent primary costing of critical carer ${ }^{20}$. The paper reports the results of a detailed activity-based costing in a hospital in Karachi, disaggregated by phase of care. We used the cost data for the ward stay phase, removing any supplies or equipment specific to the surgery, to estimate the average generic costs of a bed-day.

All bed-day costs were compared to and validated against available country-specific estimates from the published literature and from ongoing research and WHO CHOICE (see Table S2.4). Rapid literature searches were conducted on the Medline, Embase and EconLit databases on $8^{\text {th }}$ and $9^{\text {th }}$ April 2020 to identify records reporting on the costs of ICU care in each of the study countries (see table below).

Table S2.2.5.5.1 Comparison of bed-day costs by setting

\begin{tabular}{|l|l|l|l|}
\hline & \multicolumn{2}{|l|}{ Unit cost (2019 US\$) } & \\
\hline & Extrapolated & From literature & Source \\
\hline Kenya & 362.49 & 349.25 & (Lalani et al., 2018) \\
\hline India & 512.44 & 585.80 & (Mathai et al., 2015) \\
\hline South Africa & 1240.96 & 1644.27 & (Mahomed and Mahomed, 2019) \\
\hline
\end{tabular}


medRxiv preprint doi: https://doi.org/10.1101/2020.08.23.20180299; this version posted August 25, 2020. The copyright holder for this preprint (which was not certified by peer review) is the author/funder, who has granted medRxiv a license to display the preprint in perpetuity.

It is made available under a CC-BY-NC-ND 4.0 International license .

We estimated the additional costs of ICU beds compared to standard hospital beds using an ingredients-based approach to cost the equipment and supplies not present in standard hospital wards. We used the procurement price of equipment and assumed depreciation over ten (ventilators and suction pumps) or five years (all other equipment). Supply costs included central and arterial lines, ventilator tubing, and sedatives.

\section{S2.2.5.6 COVID specific costs}

Finally, we calculated the costs of personal protective equipment (PPE) per health worker per day (see Table below) and allocated a cost per PPE per minute to clinical staff. We also calculated costs of hygiene per bed day. We estimated the costs of PPE and hygiene supplies using a list of necessary supplies from a COVID-related budget from the Ministry of Health of Pakistan, which included local prices sourced by the Aga Kahn University. This was complemented for other countries using the WHO's Essential Supplies Forecasting Tool (ESFT) [7]. We divided supplies into single-use and disposable. We determined plausible quantities and useful life for supplies following clinical guidelines and expert opinion.

Table S2.2.5.6.1 PPE costs per general ward bed day and per ICU bed day

\begin{tabular}{|c|c|c|c|c|c|}
\hline Supply & Price US\$ & $\begin{array}{l}\text { Useful life } \\
\text { (days) }\end{array}$ & $\begin{array}{c}\text { Quantity per } \\
\text { day }\end{array}$ & $\begin{array}{c}\text { Total per } \\
\text { member of } \\
\text { staff per day } \\
\text { US\$ }\end{array}$ & Assumptions \\
\hline \multicolumn{6}{|l|}{$\begin{array}{l}\text { PPE for General } \\
\text { Ward }\end{array}$} \\
\hline \multicolumn{6}{|l|}{ Single Use } \\
\hline Surgical Gowns & 0.20 & 1 & 1 & 0.20 & \\
\hline Nitrile Gloves & 0.05 & 1 & 10 & 0.45 & \\
\hline Latex Gloves & 0.04 & 1 & 10 & 0.39 & \\
\hline Disposable Head & 0.03 & 1 & 4 & 0.10 & \\
\hline Shoe Covers & 0.02 & 1 & 4 & 0.06 & \\
\hline Surgical Masks & 0.08 & 1 & 10 & 0.77 & \\
\hline \multicolumn{6}{|l|}{ Reusable } \\
\hline Goggles & 11.61 & 90 & 1.5 & 0.19 & Assuming half a day for washing \\
\hline Gum Boots & 19.35 & 90 & 1.5 & 0.32 & Assuming half a day for washing \\
\hline TOTAL & & & & 250 & \\
\hline \multicolumn{6}{|l|}{ PPE for ICU } \\
\hline \multicolumn{6}{|l|}{ Single Use } \\
\hline N-95 Masks & 0.84 & 1 & 4 & 3.35 & \\
\hline Disposable apron & 0.20 & 1 & 1 & 0.20 & \\
\hline Nitrile Gloves & 0.05 & 1 & 10 & 0.45 & \\
\hline Latex Gloves & 0.04 & 1 & 10 & 0.39 & \\
\hline Disposable Head & 0.03 & 1 & 4 & 0.10 & \\
\hline Shoe Covers & 0.02 & 1 & 4 & 0.06 & \\
\hline Surgical Masks & 0.08 & 1 & 10 & 0.77 & \\
\hline \multicolumn{6}{|l|}{ Reusable } \\
\hline Face Shields & 27.81 & 5 & 1.5 & 8.34 & Assuming half a day for washing \\
\hline
\end{tabular}


medRxiv preprint doi: https://doi.org/10.1101/2020.08.23.20180299; this version posted August 25, 2020. The copyright holder for this preprint (which was not certified by peer review) is the author/funder, who has granted medRxiv a license to display the preprint in perpetuity.

It is made available under a CC-BY-NC-ND 4.0 International license .

\begin{tabular}{|l|l|l|l|l|l|}
\hline Goggles & 11.61 & 90 & 1.5 & 0.19 & Assuming half a day for washing \\
\hline Gum Boots & 19.35 & 90 & 1.5 & 0.32 & Assuming half a day for washing \\
\hline TOTAL & & & & $\mathbf{1 4 . 1 9}$ & \\
\hline
\end{tabular}

Table S2.2.5.6.2 Hygiene costs per General Ward and ICU bed day

\begin{tabular}{|c|c|c|c|c|c|}
\hline Supply & $\begin{array}{l}\text { Price USD } \\
\end{array}$ & $\begin{array}{l}\text { Useful life } \\
\text { (days) }\end{array}$ & $\begin{array}{l}\text { Quantityper } \\
\text { day }\end{array}$ & $\begin{array}{l}\text { Total per ICU bed } \\
\text { per day USD }\end{array}$ & Assumptions \\
\hline \multicolumn{6}{|l|}{ Single Use } \\
\hline Hand Sanitizers & 47.97 & 1 & 0.05 & 2.40 & $\begin{array}{l}100 \mathrm{ml} \text { use per day, price assumed to refer } \\
\text { to bottle of } 2000 \mathrm{ml}\end{array}$ \\
\hline Biohazard Bags & 0.23 & 1 & 1 & 0.23 & \\
\hline Disposable bed sheets & 1.94 & 1 & 1 & 1.94 & \\
\hline $\begin{array}{l}\text { Disposable Tissue } \\
\text { Boxes }\end{array}$ & 0.65 & 1 & 1 & 0.65 & $\begin{array}{l}1 \text { box per day, price assumed to refer to } 1 \\
\text { box }\end{array}$ \\
\hline Disposable Tissue rolls & 0.35 & 1 & 1 & 0.35 & $\begin{array}{l}1 \text { roll per day, price assumed to refer to } 1 \\
\text { roll }\end{array}$ \\
\hline $\begin{array}{l}\text { Disinfectants (1L } \\
\text { Dettol) }\end{array}$ & 3.23 & 1 & 0.25 & 0.81 & $\begin{array}{l}250 \mathrm{ml} \text { used per day, price refers to bottle } \\
\text { of } 1000 \mathrm{ml}\end{array}$ \\
\hline $\begin{array}{l}\text { Liquid Soaps }(250 \mathrm{ml} \\
\text { Dettol bottles) }\end{array}$ & 1.74 & 1 & 0.2 & 0.35 & $\begin{array}{l}50 \mathrm{ml} \text { used per day, price refers to bottle } \\
\text { of } 250 \mathrm{ml}\end{array}$ \\
\hline Ethanol (1L bottles) & 16.13 & 1 & 0.1 & 1.61 & $\begin{array}{l}100 \mathrm{ml} \text { used per day, price refers to bottle } \\
\text { of } 1000 \mathrm{ml}\end{array}$ \\
\hline Liquid Bleach & 2.58 & 1 & 0.25 & 0.65 & $\begin{array}{l}250 \mathrm{ml} \text { used per day, price assumed to } \\
\text { refer to bottle of } 1000 \mathrm{ml}\end{array}$ \\
\hline \multicolumn{6}{|l|}{ Reusable } \\
\hline Waste Bins & 15.03 & 90 & 1 & 0.17 & \\
\hline $\begin{array}{l}\text { Mackintosh bed } \\
\text { sheets }\end{array}$ & 9.68 & 90 & 1 & 0.11 & \\
\hline Mops & 2.58 & 90 & 1 & 0.03 & \\
\hline Dusters & 0.32 & 90 & 1 & 0.00 & \\
\hline TOTAL & & & & 9.28 & \\
\hline
\end{tabular}

Oxygen supplementation therapy is the main form of treatment for COVID 19. There are different methods of oxygen delivery which utilise different types of supplies, equipment and require different average levels of oxygen flow. We calculated costs for 6 types of oxygen delivery techniques and assumed a distribution across severe and critical patients according to members of our research team with clinical expertise in critical care in LMICs. See Table below.

Table S2.2.5.6.3

\begin{tabular}{|c|c|c|c|c|}
\hline \multicolumn{2}{|c|}{ Normative recommendations } & \multicolumn{3}{|c|}{ Real-world' scenario } \\
\hline Severe case & Critical case & Severe case & Criti & \\
\hline $\begin{array}{l}\text { Severe } \\
\text { pneumonia } \\
\text { (15\% of } \\
\text { COVID cases) }\end{array}$ & $\begin{array}{l}\text { Acute } \\
\text { respiratory } \\
\text { distress } \\
\text { synd rome } \\
\text { (5\% of } \\
\text { COVID } \\
\text { cases) }\end{array}$ & $\begin{array}{l}\text { Severe } \\
\text { pneumonia } \\
\text { ( } 15 \% \text { of } \\
\text { COVID cases) }\end{array}$ & $\begin{array}{l}\text { Acute respiratory dis } \\
\text { COVID cases) }\end{array}$ & ome $15 \%$ of \\
\hline General ward & ICU & General ward & General ward only & ICU \\
\hline
\end{tabular}


medRxiv preprint doi: https://doi.org/10.1101/2020.08.23.20180299; this version posted August 25, 2020. The copyright holder for this preprint (which was not certified by peer review) is the author/funder, who has granted medRxiv a license to display the preprint in perpetuity.

It is made available under a CC-BY-NC-ND 4.0 International license .

\begin{tabular}{|l|c|c|c|c|c|} 
Supplemental oxygen management type & & & & \\
\hline$\%$ ventilator & $0 \%$ & $100 \%$ & $0 \%$ & $0 \%$ & $50 \%$ \\
\hline$\%$ CPAP & $0 \%$ & $0 \%$ & $0 \%$ & $0 \%$ & $25 \%$ \\
\hline$\%$ high-flow nasal cannula & $0 \%$ & $0 \%$ & $0 \%$ & $0 \%$ & $0 \%$ \\
\hline$\%$ non-rebreather mask & $25 \%$ & $0 \%$ & $25 \%$ & $100 \%$ & $0 \%$ \\
\hline$\%$ nasal cannula & $50 \%$ & $0 \%$ & $50 \%$ & $0 \%$ & $0 \%$ \\
\hline$\%$ high-concentration mask & $25 \%$ & $0 \%$ & $25 \%$ & $0 \%$ & $67 \%$ \\
\hline$\%$ Patients in pathway & $100 \%$ & $100 \%$ & $100 \%$ & $33 \%$ & \\
\hline
\end{tabular}

S2.3 Extrapolation of unit costs in base countries to calculate unit costs across 84 LICS, LMICS and UMICS

We used the unit costs obtained in our three base countries to extrapolate unit costs to other LICS, LMICs and UMICs. We grouped countries according to income group. Costs for LICs were extrapolated using unit costs from Ethiopia, costs for LMICs were extrapolated from the unit costs from Pakistan, and those for UMICs from the unit costs from South Africa.

In order to carry out the extrapolation, each cost ingredient for each of the unit costs was classified as a tradeable good, non-tradeable good, or staff cost.

Tradeable goods are generally defined as those that can easily be traded in the international market and include goods such as medical or other supplies and medications. The unit costs for our three base countries were initially converted from each local currency into 2019 US\$ using market exchange rates. To convert the tradeable good from the base country (e.g. Ethiopia) to a 'second' country (e.g. Afghanistan) we apportioned the percentage of the unit cost that was composed of tradeable goods in 2019 US\$ from the base country to the second country.

Non-tradeable goods include buildings, heavy machinery, and other equipment. To convert these costs from a base country to a second country we used purchasing power parity (PPP) conversion rates. We multiplied the proportion of the unit cost that was defined as non-tradeable (in 2019 US\$) by the ratio of the 2019 GDP per capita (adjusted for PPP) of the second county, divided by the 2019 GDP per capita (adjusted for PPP) of the base country. Data on GDP per capita (adjusted for PPP) can be found in the World Bank database [17].

To convert staff costs from a base country to a second country we used conversion rates from Serje et al (2018) [18]. Serje et al (2018) use regression analysis on a dataset containing wages from health workers of different skill levels for 193 countries in order to predict wages by country income level relative to GDP per capita. We used the multiples per GDP per capita presented in the paper in order to convert the staff wages from the base country to the second country. See the table from Serje et al (2018) below. 
medRxiv preprint doi: https://doi.org/10.1101/2020.08.23.20180299; this version posted August 25, 2020. The copyright holder for this preprint (which was not certified by peer review) is the author/funder, who has granted medRxiv a license to display the preprint in perpetuity.

It is made available under a CC-BY-NC-ND 4.0 International license.

Table S2.3.1 Health worker earnings as a multiple of GDP per capita (Serje et al 2018)

\begin{tabular}{|l|l|l|}
\hline World bank income categories & Health worker cadre & $\begin{array}{l}\text { Average earnings index } \\
\text { (multiple of GDP per capita) }\end{array}$ \\
\hline High-income countries & Physicians & 1.9 \\
\hline & Nurses and midwives & 1.5 \\
\hline Upper-middle income countries & Other health workers & 0.9 \\
\hline & Nurses and midwives & 2.7 \\
\hline & Other health workers & 1.3 \\
\hline Lower-middle income countries & Physicians & 5.1 \\
\hline & Nurses and midwives & 4.2 \\
\hline & Other health workers & 2.4 \\
\hline Lower-income countries & Physicians & 7.8 \\
\hline & Nurses and midwives & 6.4 \\
\hline & Other health workers & 3.7 \\
\hline Global & Physicians & 4.4 \\
\hline & Nurses and midwives & 3.6 \\
\hline & Other health workers & 2.1 \\
\hline & & \\
\hline & & 2.2 \\
\hline
\end{tabular}


S2.4 Calculation of total costs per country using country-specific unit costs, modelled data on the number of cases, hospitalisations and deaths, as well as other epidemiological and economic assumptions

The unit cost in each of the 84 countries was used to calculate the total costs per activity per country. The table below explains the quantities that those unit costs were multiplied by in order to calculate the total costs per country.

Table S2.4.1

\begin{tabular}{|c|c|c|c|c|}
\hline Activity & Unit Type & Quantities per country & Value & Source \\
\hline $\begin{array}{l}\text { 1.a. Emergency Response } \\
\text { Mechanisms: National level }\end{array}$ & $\begin{array}{l}\text { Per country } \\
\text { per day }\end{array}$ & Number of working days per year & 260 & Assumption \\
\hline $\begin{array}{l}\text { 1.b. Emergency Response } \\
\text { Mechanisms: Training of health } \\
\text { staff }\end{array}$ & $\begin{array}{l}\text { One-off per } \\
\text { site }\end{array}$ & Total number of clinical sites * & $\begin{array}{l}\text { Variable per } \\
\text { Country }\end{array}$ & $\begin{array}{l}\text { Calculated by assuming } \\
\text { one site for every } 200 \\
\text { hospital beds available in } \\
\text { the country [24] }\end{array}$ \\
\hline $\begin{array}{l}\text { 2. Risk communication \& } \\
\text { comm unity engagement }\end{array}$ & $\begin{array}{l}\text { Per country } \\
\text { per day }\end{array}$ & Number of calendar days per year & 365 & $\mathrm{~N} / \mathrm{A}$ \\
\hline \multirow{4}{*}{$\begin{array}{l}\text { 3.a. Case finding, contact tracing } \\
\text { and management: Contact } \\
\text { tracing }\end{array}$} & $\begin{array}{l}\text { Per person } \\
\text { contacted }\end{array}$ & Total number of COVID 19 cases * & $\begin{array}{l}\text { Variable by } \\
\text { country }\end{array}$ & See Table S1.3.1 \\
\hline & & $\%$ cases that are symptomatic $*$ & $69 \%$ & {$[25]$} \\
\hline & & $\%$ of symptomatic cases tested $*$ & $10 \%$ & Assumption \\
\hline & & $\begin{array}{l}\text { Average number of contacts per } \\
\text { COVID19-positive case }\end{array}$ & 7 & {$[26]$} \\
\hline \multirow{4}{*}{$\begin{array}{l}\text { 3.b. Case finding, contact tracing } \\
\text { and management: Quarantine of } \\
\text { contacts }\end{array}$} & $\begin{array}{l}\text { Per person } \\
\text { quarantined }\end{array}$ & Total number of COVID 19 cases * & $\begin{array}{l}\text { Variable by } \\
\text { country }\end{array}$ & See Table S1.3.1 \\
\hline & & $\%$ cases that are symptomatic $*$ & $69 \%$ & {$[25]$} \\
\hline & & $\%$ of symptomatic cases tested $*$ & $10 \%$ & Assumption \\
\hline & & $\begin{array}{l}\text { Average number of contacts per } \\
\text { COVID19-positive case }\end{array}$ & 7 & {$[26]$} \\
\hline \multirow[t]{3}{*}{$\begin{array}{l}\text { 4.a. Surveillance: Case } \\
\text { notification }\end{array}$} & $\begin{array}{l}\text { Per positive } \\
\text { case }\end{array}$ & Total number of COVID 19 cases * & $\begin{array}{l}\text { Variable per } \\
\text { Country }\end{array}$ & See Table S1.3.1 \\
\hline & & $\%$ cases that are symptomatic $*$ & $69 \%$ & {$[25]$} \\
\hline & & $\%$ of symptomatic cases tested $*$ & $10 \%$ & Assumption \\
\hline \multirow[t]{2}{*}{$\begin{array}{l}\text { 4.b. Surveillance: Reporting } \\
\text { (national level) }\end{array}$} & $\begin{array}{l}\text { Per country } \\
\text { per day }\end{array}$ & Total number of clinical sites * & $\begin{array}{l}\text { Variable per } \\
\text { Country }\end{array}$ & $\begin{array}{l}\text { Calculated by assuming } \\
\text { one site for every } 200 \\
\text { hospital beds available in } \\
\text { the country [24] }\end{array}$ \\
\hline & & Weeks per year & 52 & $\mathrm{~N} / \mathrm{A}$ \\
\hline
\end{tabular}


medRxiv preprint doi: https://doi.org/10.1101/2020.08.23.20180299; this version posted August 25, 2020. The copyright holder for this preprint (which was not certified by peer review) is the author/funder, who has granted medRxiv a license to display the preprint in perpetuity.

It is made available under a CC-BY-NC-ND 4.0 International license.

\begin{tabular}{|c|c|c|c|c|}
\hline $\begin{array}{l}\text { 5. Public health measures: } \\
\text { Hygiene education }\end{array}$ & $\begin{array}{l}\text { Per education } \\
\text { campaign }\end{array}$ & Months per year & 12 & N/A \\
\hline \multirow[t]{7}{*}{ 6. Screening and diagnosis } & $\begin{array}{l}\text { Per person } \\
\text { screened and } \\
\text { tested }\end{array}$ & (Total number of COVID19 cases * & $\begin{array}{l}\text { Variable per } \\
\text { Country }\end{array}$ & See Table S1.3.1 \\
\hline & & $\begin{array}{l}\% \text { of cases requiring } \\
\text { hospitalisation * }\end{array}$ & $18.50 \%$ & {$[16,19]$} \\
\hline & & $\begin{array}{l}\text { Number of people tested per } \\
\text { positive case) + }\end{array}$ & 11.31 & See Table 2.4 .2 \\
\hline & & (Total number of COVID19 cases * & $\begin{array}{l}\text { Variable per } \\
\text { Country }\end{array}$ & See Table S1.3.1 \\
\hline & & $\%$ cases that are symptomatic $*$ & $69 \%$ & [25] \\
\hline & & $\%$ of symptomatic cases tested $*$ & $10 \%$ & Assumption \\
\hline & & $\begin{array}{l}\text { Number of people tested per } \\
\text { positive case) }\end{array}$ & 11.31 & See Table 2.4 .2 \\
\hline $\begin{array}{l}\text { 7.a. Case Management: Home- } \\
\text { based care }\end{array}$ & $\begin{array}{l}\text { Per person } \\
\text { requiring } \\
\text { home-based } \\
\text { care }\end{array}$ & $\begin{array}{l}\text { Proportion of borderline mild-to- } \\
\text { severe cases }\end{array}$ & $10 \%$ & Assumption \\
\hline $\begin{array}{l}\text { 7.b. Case Management: } \\
\text { Hospital-based (severe case) }\end{array}$ & $\begin{array}{l}\text { Per day of } \\
\text { hospitalisation } \\
\text { (severe case) }\end{array}$ & $\begin{array}{l}\text { Average number of days of } \\
\text { hospitalisation for severe cases }\end{array}$ & 8 & {$[16,20]$} \\
\hline $\begin{array}{l}\text { 7.c. Case Management: } \\
\text { Hospital-based (critical case) }\end{array}$ & $\begin{array}{l}\text { Per day of } \\
\text { hospitalisation } \\
\text { (critical case) }\end{array}$ & $\begin{array}{l}\text { Average number of days of } \\
\text { hospitalisation for critical cases }\end{array}$ & 10 & {$[16,19]$} \\
\hline 7.d. Case Management: Death & $\begin{array}{l}\text { Per COVID- } \\
\text { related death }\end{array}$ & $\begin{array}{l}\text { Total number of deaths from } \\
\text { COVID19 }\end{array}$ & $\begin{array}{l}\text { Variable per } \\
\text { Country }\end{array}$ & See Table S1.3.1 \\
\hline
\end{tabular}

Table 2.4.2. Test positivity rate by country and average

\begin{tabular}{|l|r|r|}
\hline \multicolumn{1}{|c|}{ Country } & \% of positive tests & Source \\
\hline South Africa & 0.169 & {$[27]$} \\
\hline Kenya & 0.103 & {$[26]$} \\
\hline Ethiopia & 0.0739 & {$[28]$} \\
\hline India & 0.0612 & {$[30]$} \\
\hline Pakistan & 0.0351 & \\
\hline Average & $\mathbf{0 . 0 8 8 4 4}$ & \\
\hline
\end{tabular}

S2.5 Calculation of country-specific per capita costs, as well as per capita costs as a proportion of gross domestic product (GDP) per capita and various measures of health expenditure per capita. 
medRxiv preprint doi: https://doi.org/10.1101/2020.08.23.20180299; this version posted August 25, 2020. The copyright holder for this preprint (which was not certified by peer review) is the author/funder, who has granted medRxiv a license to display the preprint in perpetuity.

It is made available under a CC-BY-NC-ND 4.0 International license.

Total costs per country were used to calculate the COVID-19-related costs per capita per country per scenario by dividing the total costs by the population of the country [17]. The cost per capita was then calculated as a proportion of GDP per capita [17] and three measures of health expenditure per capita [31]: 1) total health expenditure including out-of-pocket payments, 2) total health expenditure excluding out-of-pocket payments, and 3) government health spending per capita. Data on GDP per capita and health expenditure per capita per country can be found in the table below.

\section{S.2.5.1 GDP, Health Spending and Government Health Spending}

\begin{tabular}{|c|c|c|c|c|c|c|c|c|c|c|}
\hline \multirow[b]{2}{*}{ Afghanistan } & \multirow{2}{*}{$\begin{array}{l}\begin{array}{c}\text { Country } \\
\text { income } \\
\text { classification }\end{array} \\
\text { LIC }\end{array}$} & \multirow{2}{*}{$\begin{array}{c}\begin{array}{c}\text { Total } \\
\text { population } \\
\text { per country }\end{array} \\
\\
37,172,386\end{array}$} & \multicolumn{2}{|c|}{$\begin{array}{l}\text { Gross Domestic } \\
\text { Product per } \\
\text { Capita (Nominal) } \\
\text { (US\$) }\end{array}$} & \multicolumn{2}{|c|}{$\begin{array}{l}\text { Total Health } \\
\text { Spending per } \\
\text { Capita (including } \\
\text { out-of-pocket } \\
\text { spending) (US\$) }\end{array}$} & \multicolumn{2}{|c|}{$\begin{array}{l}\text { Total Health } \\
\text { Spending per } \\
\text { Capita (excluding } \\
\text { out-of-pocket } \\
\text { spending) (US\$) }\end{array}$} & \multicolumn{2}{|c|}{$\begin{array}{l}\text { Government } \\
\text { Health Spending } \\
\text { per Capita (US\$) }\end{array}$} \\
\hline & & & $\$$ & 520.90 & $\$$ & 57.25 & $\$$ & 101.56 & $\$$ & 2.94 \\
\hline Albania & UMIC & $2,866,376$ & $\$$ & $5,268.85$ & $\$$ & 271.54 & $\$$ & 428.99 & $\$$ & 112.30 \\
\hline Algeria & UMIC & $42,228,429$ & $\$$ & $4,114.72$ & $\$$ & 260.41 & $\$$ & 340.84 & $\$$ & 176.27 \\
\hline American Samoa & UMIC & 55,465 & $\$$ & $11,466.69$ & $\$$ & - & $\$$ & - & $\$$ & - \\
\hline Andorra & $\mathrm{HIC}$ & 77,006 & $\$$ & $42,029.76$ & $\$$ & $3,834.73$ & $\$$ & $5,433.69$ & $\$$ & $1,884.00$ \\
\hline Angola & LMIC & $30,809,762$ & $\$$ & $3,432.39$ & $\$$ & 95.22 & $\$$ & 128.75 & $\$$ & 42.03 \\
\hline Antigua and Barbuda & $\mathrm{HIC}$ & 96,286 & $\$$ & $16,726.98$ & $\$$ & 623.11 & $\$$ & 823.69 & $\$$ & 377.32 \\
\hline Argentina & UMIC & $44,494,502$ & $\$$ & $11,683.95$ & $\$$ & 955.20 & $\$$ & $1,106.11$ & $\$$ & 710.94 \\
\hline Armenia & UMIC & $2,951,776$ & $\$$ & $4,212.07$ & $\$$ & 358.84 & $\$$ & 648.24 & $\$$ & 59.04 \\
\hline Aruba & $\mathrm{HIC}$ & 105,845 & $\$$ & $25,630.27$ & $\$$ & - & $\$$ & - & $\$$ & - \\
\hline Australia & $\mathrm{HIC}$ & $24,992,369$ & $\$$ & $57,373.69$ & $\$$ & $5,002.36$ & $\$$ & $5,949.99$ & $\$$ & $3,416.98$ \\
\hline Austria & $\mathrm{HIC}$ & $8,847,037$ & $\$$ & $51,461.95$ & $\$$ & $4,688.28$ & $\$$ & $5,575.29$ & $\$$ & $3,399.49$ \\
\hline Azerbaijan & UMIC & $9,942,334$ & $\$$ & $4,721.18$ & $\$$ & 268.16 & $\$$ & 479.78 & $\$$ & 53.53 \\
\hline Bahamas, The & $\mathrm{HIC}$ & 385,640 & $\$$ & $32,217.87$ & $\$$ & $1,835.23$ & $\$$ & $2,343.88$ & $\$$ & 915.06 \\
\hline Bahrain & $\mathrm{HIC}$ & $1,569,439$ & $\$$ & $24,050.76$ & $\$$ & $1,099.36$ & $\$$ & $1,407.05$ & $\$$ & 674.61 \\
\hline Bangladesh & LMIC & $\begin{array}{r}161,356,03 \\
9\end{array}$ & $\$$ & $1,698.26$ & $\$$ & 34.22 & $\$$ & 58.82 & $\$$ & 6.14 \\
\hline Barbados & $\mathrm{HIC}$ & 286,641 & $\$$ & $17,949.28$ & $\$$ & $1,163.84$ & $\$$ & $1,689.63$ & $\$$ & 534.05 \\
\hline Belarus & UMIC & $9,485,386$ & $\$$ & $6,289.94$ & $\$$ & 317.99 & $\$$ & 431.83 & $\$$ & 195.40 \\
\hline Belgium & $\mathrm{HIC}$ & $11,422,068$ & $\$$ & $47,518.64$ & $\$$ & $4,149.39$ & $\$$ & $4,807.43$ & $\$$ & $3,489.87$ \\
\hline Belize & UMIC & 383,071 & $\$$ & $4,884.74$ & $\$$ & 303.60 & $\$$ & 373.04 & $\$$ & 201.37 \\
\hline Benin & LIC & $11,485,048$ & $\$$ & 901.54 & $\$$ & 30.40 & $\$$ & 43.62 & $\$$ & 6.24 \\
\hline Bermuda & $\mathrm{HIC}$ & 63,968 & $\$$ & $85,748.07$ & $\$$ & & $\$$ & - & $\$$ & - \\
\hline Bhutan & LMIC & 754,394 & $\$$ & $3,243.23$ & $\$$ & 91.34 & $\$$ & 109.72 & $\$$ & 67.55 \\
\hline Bolivia & LMIC & $11,353,142$ & $\$$ & $3,548.59$ & $\$$ & 213.02 & $\$$ & 272.71 & $\$$ & 139.95 \\
\hline Bosnia and Herzegovina & UMIC & $3,323,929$ & $\$$ & $6,065.67$ & $\$$ & 443.78 & $\$$ & 571.07 & $\$$ & 314.14 \\
\hline Botswana & UMIC & $2,254,126$ & $\$$ & $8,258.64$ & $\$$ & 379.92 & $\$$ & 399.86 & $\$$ & 212.50 \\
\hline Brazil & UMIC & $209,469,33$ & $\$$ & $8,920.76$ & $\$$ & $1,015.93$ & $\$$ & $1,458.48$ & $\$$ & 337.53 \\
\hline
\end{tabular}


medRxiv preprint doi: https://doi.org/10.1101/2020.08.23.20180299; this version posted August 25, 2020. The copyright holder for this preprint (which was not certified by peer review) is the author/funder, who has granted medRxiv a license to display the preprint in perpetuity.

It is made available under a CC-BY-NC-ND 4.0 International license.

\begin{tabular}{|c|c|c|c|c|c|c|c|c|c|c|}
\hline British Virgin Islands & HIC & 29,802 & $\$$ & - & $\$$ & - & $\$$ & - & $\$$ & - \\
\hline Brunei Darussalam & HIC & 428,962 & $\$$ & $31,628.33$ & $\$$ & 630.63 & $\$$ & 662.61 & $\$$ & 598.65 \\
\hline Bulgaria & UMIC & $7,024,216$ & $\$$ & $9,272.63$ & $\$$ & 612.48 & $\$$ & 906.19 & $\$$ & 309.69 \\
\hline Burkina Faso & LIC & $19,751,535$ & $\$$ & 715.12 & $\$$ & 40.94 & $\$$ & 53.79 & $\$$ & 16.42 \\
\hline Burundi & LIC & $11,175,378$ & $\$$ & 271.75 & $\$$ & 18.46 & $\$$ & 24.10 & $\$$ & 5.38 \\
\hline Cabo Verde & LMIC & 543,767 & $\$$ & $3,635.41$ & $\$$ & 159.07 & $\$$ & 200.46 & $\$$ & 90.33 \\
\hline Cambodia & LMIC & $16,249,798$ & $\$$ & $1,510.32$ & $\$$ & 77.67 & $\$$ & 123.16 & $\$$ & 16.94 \\
\hline Cameroon & LMIC & $25,216,237$ & $\$$ & $1,533.74$ & $\$$ & 64.47 & $\$$ & 109.27 & $\$$ & 8.60 \\
\hline Canada & HIC & $37,058,856$ & $\$$ & $46,232.99$ & $\$$ & $4,458.21$ & $\$$ & $5,109.89$ & $\$$ & $3,274.29$ \\
\hline Cayman Islands & HIC & 64,174 & $\$$ & $81,124.51$ & $\$$ & - & $\$$ & - & $\$$ & - \\
\hline Central African Republic & LIC & $4,666,377$ & $\$$ & 475.72 & $\$$ & 16.36 & $\$$ & 23.41 & $\$$ & 2.43 \\
\hline Chad & LIC & $15,477,751$ & $\$$ & 728.34 & $\$$ & 31.69 & $\$$ & 51.06 & $\$$ & 5.98 \\
\hline Channel Islands & HIC & 170,499 & $\$$ & $74,462.65$ & $\$$ & & $\$$ & & $\$$ & \\
\hline Chile & HIC & $18,729,160$ & $\$$ & $15,923.36$ & $\$$ & $1,190.55$ & $\$$ & $1,604.50$ & $\$$ & 696.10 \\
\hline China & UMIC & $\begin{array}{r}1,392,730,0 \\
\infty\end{array}$ & $\$$ & $9,770.85$ & $\$$ & 398.33 & $\$$ & 541.36 & $\$$ & 231.09 \\
\hline Colombia & UMIC & $49,648,685$ & $\$$ & $6,667.79$ & $\$$ & 340.37 & $\$$ & 408.97 & $\$$ & 215.85 \\
\hline Comoros & LMIC & 832,322 & $\$$ & $1,415.26$ & $\$$ & 59.00 & $\$$ & 102.15 & $\$$ & 8.59 \\
\hline Congo, Dem. Rep. & LIC & $84,068,091$ & $\$$ & 561.78 & $\$$ & 20.52 & $\$$ & 28.20 & $\$$ & 2.51 \\
\hline Congo, Rep. & LMIC & $5,244,363$ & $\$$ & $2,147.77$ & $\$$ & 70.38 & $\$$ & 105.37 & $\$$ & 29.75 \\
\hline Costa Rica & UMIC & $4,999,441$ & $\$$ & $12,027.37$ & $\$$ & 888.85 & $\$$ & $1,085.68$ & $\$$ & 664.48 \\
\hline Cote d'Ivoire & LMIC & $25,069,229$ & $\$$ & $1,715.53$ & $\$$ & 67.57 & $\$$ & 94.70 & $\$$ & 17.41 \\
\hline Croatia & HIC & $4,089,400$ & $\$$ & $14,909.69$ & $\$$ & 884.49 & $\$$ & $1,020.36$ & $\$$ & 692.08 \\
\hline Cuba & UMIC & $11,338,138$ & $\$$ & $8,821.82$ & $\$$ & 970.65 & $\$$ & $1,070.70$ & $\$$ & 869.70 \\
\hline Curacao & HIC & 159,849 & $\$$ & $19,567.89$ & $\$$ & - & $\$$ & - & $\$$ & - \\
\hline Cyprus & HIC & $1,189,265$ & $\$$ & $28,159.30$ & $\$$ & $1,634.43$ & $\$$ & $2,368.67$ & $\$$ & 689.75 \\
\hline Czech Republic & HIC & $10,625,695$ & $\$$ & $23,078.57$ & $\$$ & $1,321.62$ & $\$$ & $1,520.18$ & $\$$ & $1,082.12$ \\
\hline Denmark & HIC & $5,797,446$ & $\$$ & $61,350.35$ & $\$$ & $5,565.59$ & $\$$ & $6,328.77$ & $\$$ & $4,681.91$ \\
\hline Djibouti & LMIC & 958,920 & $\$$ & $3,082.54$ & $\$$ & 70.19 & $\$$ & 88.28 & $\$$ & 32.13 \\
\hline Dominica & UMIC & 71,625 & $\$$ & $7,691.35$ & $\$$ & 419.37 & $\$$ & 541.60 & $\$$ & 269.47 \\
\hline Dominican Republic & UMIC & $10,627,165$ & $\$$ & $8,050.63$ & $\$$ & 414.18 & $\$$ & 598.97 & $\$$ & 189.38 \\
\hline Ecuador & UMIC & $17,084,357$ & $\$$ & $6,344.87$ & $\$$ & 504.78 & $\$$ & 709.13 & $\$$ & 258.41 \\
\hline Egypt, Arab Rep. & LMIC & $98,423,595$ & $\$$ & $2,549.13$ & $\$$ & 130.99 & $\$$ & 212.18 & $\$$ & 38.38 \\
\hline El Salvador & LMIC & $6,420,744$ & $\$$ & $4,058.25$ & $\$$ & 293.87 & $\$$ & 373.69 & $\$$ & 189.45 \\
\hline Equatorial Guinea & UMIC & $1,308,974$ & $\$$ & $10,261.76$ & $\$$ & 281.37 & $\$$ & 486.31 & $\$$ & 66.16 \\
\hline Eritrea & LIC & .. & $\$$ & 811.38 & $\$$ & 29.89 & $\$$ & 47.54 & $\$$ & 8.73 \\
\hline Estonia & $\mathrm{HIC}$ & $1,320,884$ & $\$$ & $23,266.35$ & $\$$ & $1,185.33$ & $\$$ & $1,454.24$ & $\$$ & 893.89 \\
\hline Eswatini & LMIC & $1,136,191$ & $\$$ & $4,145.97$ & $\$$ & 220.59 & $\$$ & 242.42 & $\$$ & 152.88 \\
\hline Ethiopia & LIC & $\begin{array}{r}109,224,55 \\
9\end{array}$ & $\$$ & 772.31 & $\$$ & 27.52 & $\$$ & 37.82 & $\$$ & 7.60 \\
\hline
\end{tabular}


medRxiv preprint doi: https://doi.org/10.1101/2020.08.23.20180299; this version posted August 25, 2020. The copyright holder for this preprint (which was not certified by peer review) is the author/funder, who has granted medRxiv a license to display the preprint in perpetuity.

It is made available under a CC-BY-NC-ND 4.0 International license.

\begin{tabular}{|c|c|c|c|c|c|c|c|c|c|c|}
\hline Faroe Islands & HIC & 48,497 & $\$$ & $55,822.91$ & $\$$ & - & $\$$ & - & $\$$ & - \\
\hline Fiji & UMIC & 883,483 & $\$$ & $6,266.97$ & $\$$ & 179.91 & $\$$ & 217.04 & $\$$ & 114.97 \\
\hline Finland & $\mathrm{HIC}$ & $5,518,050$ & $\$$ & $50,152.34$ & $\$$ & $4,117.26$ & $\$$ & $4,955.28$ & $\$$ & $3,185.11$ \\
\hline France & $\mathrm{HIC}$ & $66,987,244$ & $\$$ & $41,463.64$ & $\$$ & $4,263.36$ & $\$$ & $4,679.31$ & $\$$ & $3,534.15$ \\
\hline French Polynesia & $\mathrm{HIC}$ & 277,679 & $\$$ & $14,323.82$ & $\$$ & & $\$$ & - & $\$$ & \\
\hline Gabon & UMIC & $2,119,275$ & $\$$ & $7,952.53$ & $\$$ & 220.35 & $\$$ & 269.96 & $\$$ & 142.32 \\
\hline Gambia, The & LIC & $2,280,102$ & $\$$ & 716.12 & $\$$ & 20.93 & $\$$ & 25.87 & $\$$ & 3.89 \\
\hline Georgia & UMIC & $3,731,000$ & $\$$ & $4,717.14$ & $\$$ & 308.03 & $\$$ & 479.29 & $\$$ & 112.59 \\
\hline Germany & $\mathrm{HIC}$ & $82,927,922$ & $\$$ & $47,603.03$ & $\$$ & $4,714.26$ & $\$$ & $5,299.28$ & $\$$ & $3,991.60$ \\
\hline Ghana & LMIC & $29,767,108$ & $\$$ & $2,202.31$ & $\$$ & 67.51 & $\$$ & 93.04 & $\$$ & 25.89 \\
\hline Gibraltar & $\mathrm{HIC}$ & 33,718 & $\$$ & - & $\$$ & - & $\$$ & - & $\$$ & - \\
\hline Greece & $\mathrm{HIC}$ & $10,727,668$ & $\$$ & $20,324.25$ & $\$$ & $1,510.67$ & $\$$ & $2,029.49$ & $\$$ & 917.64 \\
\hline Greenland & $\mathrm{HIC}$ & 56,025 & $\$$ & $48,181.87$ & $\$$ & & $\$$ & - & $\$$ & \\
\hline Grenada & UMIC & 111,454 & $\$$ & $10,640.50$ & $\$$ & 516.42 & $\$$ & 814.79 & $\$$ & 213.49 \\
\hline Guam & $\mathrm{HIC}$ & 165,768 & $\$$ & $35,712.56$ & $\$$ & & $\$$ & - & $\$$ & - \\
\hline Guatemala & UMIC & $17,247,807$ & $\$$ & $4,549.01$ & $\$$ & 241.35 & $\$$ & 370.09 & $\$$ & 89.82 \\
\hline Guinea & LIC & $12,414,318$ & $\$$ & 878.60 & $\$$ & 37.46 & $\$$ & 56.10 & $\$$ & 4.61 \\
\hline Guinea-Bissau & LIC & $1,874,309$ & $\$$ & 777.97 & $\$$ & 39.05 & $\$$ & 52.88 & $\$$ & 17.28 \\
\hline Guyana & UMIC & 779,004 & $\$$ & $4,979.00$ & $\$$ & 192.27 & $\$$ & 259.68 & $\$$ & 113.32 \\
\hline Haiti & LIC & $11,123,176$ & $\$$ & 868.34 & $\$$ & 37.72 & $\$$ & 53.47 & $\$$ & 5.79 \\
\hline Honduras & LMIC & $9,587,522$ & $\$$ & $2,500.11$ & $\$$ & 199.55 & $\$$ & 289.38 & $\$$ & 91.61 \\
\hline Hong Kong SAR, China & $\mathrm{HIC}$ & $7,451,000$ & $\$$ & $48,675.62$ & $\$$ & 312.58 & $\$$ & 312.58 & $\$$ & 197.84 \\
\hline Hungary & $\mathrm{HIC}$ & $9,768,785$ & $\$$ & $16,161.98$ & $\$$ & 942.59 & $\$$ & $1,222.53$ & $\$$ & 620.82 \\
\hline Iceland & $\mathrm{HIC}$ & 353,574 & $\$$ & $73,191.12$ & $\$$ & $5,063.61$ & $\$$ & $5,917.79$ & $\$$ & $4,127.35$ \\
\hline India & LMIC & $\begin{array}{r}1,352,617,3 \\
28\end{array}$ & $\$$ & $2,009.98$ & $\$$ & 62.72 & $\$$ & 103.22 & $\$$ & 15.95 \\
\hline Indonesia & LMIC & $\begin{array}{r}267,663,43 \\
5\end{array}$ & $\$$ & $3,893.60$ & $\$$ & 111.55 & $\$$ & 153.20 & $\$$ & 49.90 \\
\hline Iran, Islamic Rep. & UMIC & $81,800,269$ & $\$$ & $5,627.75$ & $\$$ & 415.39 & $\$$ & 576.54 & $\$$ & 226.49 \\
\hline Iraq & UMIC & $38,433,600$ & $\$$ & $5,834.17$ & $\$$ & 152.64 & $\$$ & 272.44 & $\$$ & 32.24 \\
\hline Ireland & $\mathrm{HIC}$ & $4,853,506$ & $\$$ & $78,806.43$ & $\$$ & $4,758.59$ & $\$$ & $5,376.76$ & $\$$ & $3,429.35$ \\
\hline Isle of Man & $\mathrm{HIC}$ & 84,077 & $\$$ & $80,989.17$ & $\$$ & & $\$$ & - & $\$$ & - \\
\hline Israel & $\mathrm{HIC}$ & $8,883,800$ & $\$$ & $41,715.03$ & $\$$ & $2,837.14$ & $\$$ & $3,488.89$ & $\$$ & $1,773.23$ \\
\hline Italy & $\mathrm{HIC}$ & $60,431,283$ & $\$$ & $34,483.20$ & $\$$ & $2,738.71$ & $\$$ & $3,371.50$ & $\$$ & $2,039.36$ \\
\hline Jamaica & UMIC & $2,934,855$ & $\$$ & $5,354.24$ & $\$$ & 296.25 & $\$$ & 362.59 & $\$$ & 179.41 \\
\hline Japan & $\mathrm{HIC}$ & $\begin{array}{r}126,529,10 \\
0\end{array}$ & $\$$ & $39,289.96$ & $\$$ & $4,233.03$ & $\$$ & $4,802.47$ & $\$$ & $3,538.21$ \\
\hline Jordan & UMIC & $9,956,011$ & $\$$ & $4,241.79$ & $\$$ & 223.54 & $\$$ & 286.09 & $\$$ & 141.34 \\
\hline Kazakhstan & UMIC & $18,276,499$ & $\$$ & $9,812.60$ & $\$$ & 262.01 & $\$$ & 355.18 & $\$$ & 154.04 \\
\hline Kenya & LMIC & $51,393,010$ & $\$$ & $1,710.51$ & $\$$ & 66.21 & $\$$ & 84.56 & $\$$ & 23.95 \\
\hline Kiribati & LMIC & 115,847 & $\$$ & $1,625.29$ & $\$$ & 188.05 & $\$$ & 188.22 & $\$$ & 115.62 \\
\hline Korea, Dem. People's Rep. & LIC & $25,549,819$ & $\$$ & - & $\$$ & - & $\$$ & - & $\$$ & - \\
\hline
\end{tabular}


medRxiv preprint doi: https://doi.org/10.1101/2020.08.23.20180299; this version posted August 25, 2020. The copyright holder for this preprint (which was not certified by peer review) is the author/funder, who has granted medRxiv a license to display the preprint in perpetuity.

It is made available under a CC-BY-NC-ND 4.0 International license.

\begin{tabular}{|c|c|c|c|c|c|c|c|c|c|c|}
\hline Korea, Rep. & $\mathrm{HIC}$ & $51,635,256$ & $\$$ & $31,362.75$ & $\$$ & $2,043.86$ & $\$$ & $2,724.68$ & $\$$ & $1,209.04$ \\
\hline Kosovo & UMIC & $1,845,300$ & $\$$ & $4,302.28$ & $\$$ & - & $\$$ & - & $\$$ & - \\
\hline Kuwait & $\mathrm{HIC}$ & $4,137,309$ & $\$$ & $33,994.41$ & $\$$ & $1,068.32$ & $\$$ & $1,240.44$ & $\$$ & 896.20 \\
\hline Kyrgyz Republic & LMIC & $6,315,800$ & $\$$ & $1,281.36$ & $\$$ & 72.88 & $\$$ & 114.85 & $\$$ & 28.50 \\
\hline Lao PDR & LMIC & $7,061,507$ & $\$$ & $2,542.49$ & $\$$ & 55.21 & $\$$ & 80.86 & $\$$ & 17.89 \\
\hline Latvia & $\mathrm{HIC}$ & $1,926,542$ & $\$$ & $17,860.62$ & $\$$ & 874.20 & $\$$ & $1,263.72$ & $\$$ & 477.60 \\
\hline Lebanon & UMIC & $6,848,925$ & $\$$ & $8,269.79$ & $\$$ & 662.13 & $\$$ & 874.91 & $\$$ & 345.18 \\
\hline Lesotho & LMIC & $2,108,132$ & $\$$ & $1,299.15$ & $\$$ & 85.52 & $\$$ & 101.67 & $\$$ & 54.57 \\
\hline Liberia & LIC & $4,818,977$ & $\$$ & 677.32 & $\$$ & 68.31 & $\$$ & 100.60 & $\$$ & 9.73 \\
\hline Libya & UMIC & $6,678,567$ & $\$$ & $7,241.70$ & $\$$ & - & $\$$ & 114.62 & $\$$ & - \\
\hline Liechtenstein & $\mathrm{HIC}$ & 37,910 & $\$$ & $165,028.25$ & $\$$ & - & $\$$ & - & $\$$ & - \\
\hline Lithuania & $\mathrm{HIC}$ & $2,789,533$ & $\$$ & $19,153.41$ & $\$$ & 987.95 & $\$$ & $1,307.45$ & $\$$ & 648.12 \\
\hline Luxembourg & $\mathrm{HIC}$ & 607,728 & $\$$ & $116,639.89$ & $\$$ & $6,271.44$ & $\$$ & $6,975.98$ & $\$$ & $5,092.72$ \\
\hline Macao SAR, China & $\mathrm{HIC}$ & 631,636 & $\$$ & $87,208.54$ & $\$$ & - & $\$$ & - & $\$$ & - \\
\hline Madagascar & LIC & $26,262,368$ & $\$$ & 527.50 & $\$$ & 24.12 & $\$$ & 29.51 & $\$$ & 11.49 \\
\hline Malawi & LIC & $18,143,315$ & $\$$ & 389.40 & $\$$ & 29.59 & $\$$ & 32.96 & $\$$ & 8.30 \\
\hline Malaysia & UMIC & $31,528,585$ & $\$$ & $11,373.23$ & $\$$ & 361.52 & $\$$ & 497.44 & $\$$ & 182.47 \\
\hline Maldives & UMIC & 515,696 & $\$$ & $10,330.62$ & $\$$ & $1,047.84$ & $\$$ & $1,247.99$ & $\$$ & 760.31 \\
\hline Mali & LIC & $19,077,690$ & $\$$ & 899.66 & $\$$ & 29.79 & $\$$ & 40.30 & $\$$ & 9.41 \\
\hline Malta & $\mathrm{HIC}$ & 483,530 & $\$$ & $30,098.28$ & $\$$ & $2,327.78$ & $\$$ & $3,137.27$ & $\$$ & $1,469.08$ \\
\hline Marshall Islands & UMIC & 58,413 & $\$$ & $3,788.16$ & $\$$ & 851.34 & $\$$ & 928.00 & $\$$ & 448.16 \\
\hline Mauritania & LMIC & $4,403,319$ & $\$$ & $1,188.83$ & $\$$ & 46.77 & $\$$ & 70.58 & $\$$ & 17.14 \\
\hline Mauritius & UMIC & $1,265,303$ & $\$$ & $11,238.69$ & $\$$ & 553.10 & $\$$ & 819.47 & $\$$ & 243.98 \\
\hline Mexico & UMIC & $\begin{array}{r}126,190,78 \\
8\end{array}$ & $\$$ & $9,673.44$ & $\$$ & 461.79 & $\$$ & 648.25 & $\$$ & 240.81 \\
\hline Micronesia, Fed. Sts. & LMIC & 112,640 & $\$$ & $3,568.29$ & $\$$ & 386.69 & $\$$ & 396.86 & $\$$ & 107.66 \\
\hline Moldova & LMIC & $3,545,883$ & $\$$ & $3,227.31$ & $\$$ & 171.19 & $\$$ & 250.43 & $\$$ & 83.53 \\
\hline Monaco & $\mathrm{HIC}$ & 38,682 & $\$$ & $185,741.28$ & $\$$ & $2,939.84$ & $\$$ & $3,147.43$ & $\$$ & $2,338.81$ \\
\hline Mongolia & LMIC & $3,170,208$ & $\$$ & $4,121.73$ & $\$$ & 140.71 & $\$$ & 191.18 & $\$$ & 79.75 \\
\hline Montenegro & UMIC & 622,345 & $\$$ & $8,844.24$ & $\$$ & 531.73 & $\$$ & 659.80 & $\$$ & 398.90 \\
\hline Morocco & LMIC & $36,029,138$ & $\$$ & $3,237.88$ & $\$$ & 171.45 & $\$$ & 254.80 & $\$$ & 80.33 \\
\hline Mozambique & LIC & $29,495,962$ & $\$$ & 498.96 & $\$$ & 19.21 & $\$$ & 20.68 & $\$$ & 10.25 \\
\hline Myanmar & LMIC & $53,708,395$ & $\$$ & $1,325.95$ & $\$$ & 62.11 & $\$$ & 108.06 & $\$$ & 12.48 \\
\hline Namibia & UMIC & $2,448,255$ & $\$$ & $5,931.45$ & $\$$ & 402.76 & $\$$ & 433.84 & $\$$ & 249.29 \\
\hline Nauru & UMIC & 12,704 & $\$$ & $9,888.89$ & $\$$ & $1,012.35$ & $\$$ & $1,024.03$ & $\$$ & 614.59 \\
\hline Nepal & LIC & $28,087,871$ & $\$$ & $1,033.91$ & $\$$ & 45.45 & $\$$ & 70.65 & $\$$ & 8.44 \\
\hline Netherlands & $\mathrm{HIC}$ & $17,231,017$ & $\$$ & $53,024.06$ & $\$$ & $4,742.03$ & $\$$ & $5,285.06$ & $\$$ & $3,839.93$ \\
\hline New Caledonia & $\mathrm{HIC}$ & 284,060 & $\$$ & $12,579.60$ & $\$$ & - & $\$$ & - & $\$$ & - \\
\hline New Zealand & $\mathrm{HIC}$ & $4,885,500$ & $\$$ & $41,945.33$ & $\$$ & $3,745.22$ & $\$$ & $4,253.65$ & $\$$ & $2,945.76$ \\
\hline Nicaragua & LMIC & $6,465,513$ & $\$$ & $2,028.89$ & $\$$ & 188.16 & $\$$ & 248.79 & $\$$ & 115.43 \\
\hline Niger & LIC & $22,442,948$ & $\$$ & 413.98 & $\$$ & 22.68 & $\$$ & 35.96 & $\$$ & 5.51 \\
\hline
\end{tabular}


medRxiv preprint doi: https://doi.org/10.1101/2020.08.23.20180299; this version posted August 25, 2020. The copyright holder for this preprint (which was not certified by peer review) is the author/funder, who has granted medRxiv a license to display the preprint in perpetuity.

It is made available under a CC-BY-NC-ND 4.0 International license.

\begin{tabular}{|c|c|c|c|c|c|c|c|c|c|c|}
\hline Nigeria & LMIC & $\begin{array}{r}195,874,74 \\
0\end{array}$ & $\$$ & $2,028.18$ & $\$$ & 79.34 & $\$$ & 139.01 & $\$$ & 10.33 \\
\hline North Macedonia & UMIC & $2,082,958$ & $\$$ & $6,083.72$ & $\$$ & 327.84 & $\$$ & 443.93 & $\$$ & 208.40 \\
\hline Northern Mariana Islands & HIC & 56,882 & $\$$ & $23,258.68$ & $\$$ & - & $\$$ & - & $\$$ & - \\
\hline Norway & HIC & $5,314,336$ & $\$$ & $81,697.25$ & $\$$ & $7,477.90$ & $\$$ & $8,563.66$ & $\$$ & $6,365.95$ \\
\hline Oman & HIC & $4,829,483$ & $\$$ & $16,415.16$ & $\$$ & 648.28 & $\$$ & 686.61 & $\$$ & 577.88 \\
\hline Pakistan & LMIC & $\begin{array}{r}212,215,03 \\
0\end{array}$ & $\$$ & $1,482.40$ & $\$$ & 39.58 & $\$$ & 65.40 & $\$$ & 11.04 \\
\hline Palau & HIC & 17,907 & $\$$ & $15,859.43$ & $\$$ & $1,674.09$ & $\$$ & $1,916.07$ & $\$$ & $1,000.51$ \\
\hline Panama & HIC & $4,176,873$ & $\$$ & $15,575.07$ & $\$$ & $1,040.67$ & $\$$ & $1,326.08$ & $\$$ & 682.70 \\
\hline Papua New Guinea & LMIC & $8,606,316$ & $\$$ & $2,730.27$ & $\$$ & 55.15 & $\$$ & 59.48 & $\$$ & 38.61 \\
\hline Paraguay & UMIC & $6,956,071$ & $\$$ & $5,821.81$ & $\$$ & 327.24 & $\$$ & 451.12 & $\$$ & 168.93 \\
\hline Peru & UMIC & $31,989,256$ & $\$$ & $6,941.24$ & $\$$ & 316.44 & $\$$ & 405.95 & $\$$ & 202.75 \\
\hline Philippines & LMIC & $\begin{array}{r}106,651,92 \\
2\end{array}$ & $\$$ & $3,102.71$ & $\$$ & 129.43 & $\$$ & 199.25 & $\$$ & 40.83 \\
\hline Poland & HIC & $37,978,548$ & $\$$ & $15,420.91$ & $\$$ & 809.01 & $\$$ & 994.64 & $\$$ & 563.89 \\
\hline Portugal & HIC & $10,281,762$ & $\$$ & $23,407.91$ & $\$$ & $1,800.86$ & $\$$ & $2,300.63$ & $\$$ & $1,194.94$ \\
\hline Puerto Rico & HIC & $3,195,153$ & $\$$ & $31,651.35$ & $\$$ & - & $\$$ & - & $\$$ & - \\
\hline Qatar & HIC & $2,781,677$ & $\$$ & $68,793.78$ & $\$$ & $1,827.06$ & $\$$ & $1,983.29$ & $\$$ & $1,491.39$ \\
\hline Romania & UMIC & $19,473,936$ & $\$$ & $12,301.19$ & $\$$ & 476.37 & $\$$ & 575.21 & $\$$ & 372.46 \\
\hline Russian Federation & UMIC & $\begin{array}{r}144,478,05 \\
0\end{array}$ & $\$$ & $11,288.87$ & $\$$ & 469.13 & $\$$ & 659.06 & $\$$ & 267.17 \\
\hline Rwanda & LIC & $12,301,939$ & $\$$ & 772.94 & $\$$ & 48.08 & $\$$ & 51.14 & $\$$ & 16.29 \\
\hline Samoa & UMIC & 196,130 & $\$$ & $4,183.41$ & $\$$ & 227.15 & $\$$ & 254.14 & $\$$ & 173.50 \\
\hline San Marino & HIC & 33,785 & $\$$ & $48,494.55$ & $\$$ & $3,012.79$ & $\$$ & $3,578.91$ & $\$$ & $2,421.99$ \\
\hline Sao Tome and Principe & LMIC & 211,028 & $\$$ & $2,001.14$ & $\$$ & 105.13 & $\$$ & 120.28 & $\$$ & 41.94 \\
\hline Saudi Arabia & HIC & $33,699,947$ & $\$$ & $23,338.96$ & $\$$ & $1,147.33$ & $\$$ & $1,311.91$ & $\$$ & 777.91 \\
\hline Senegal & LMIC & $15,854,360$ & $\$$ & $1,521.95$ & $\$$ & 52.61 & $\$$ & 79.84 & $\$$ & 18.19 \\
\hline Serbia & UMIC & $6,982,084$ & $\$$ & $7,246.73$ & $\$$ & 494.42 & $\$$ & 694.68 & $\$$ & 286.79 \\
\hline Seychelles & HIC & 96,762 & $\$$ & $16,433.94$ & $\$$ & 596.92 & $\$$ & 609.36 & $\$$ & 572.88 \\
\hline Sierra Leone & LIC & $7,650,154$ & $\$$ & 533.99 & $\$$ & 86.31 & $\$$ & 122.17 & $\$$ & 9.64 \\
\hline Singapore & HIC & $5,638,676$ & $\$$ & $64,581.94$ & $\$$ & $2,462.39$ & $\$$ & $3,230.00$ & $\$$ & $1,342.62$ \\
\hline Sint Maarten (Dutch part) & HIC & 40,654 & $\$$ & - & $\$$ & & $\$$ & - & $\$$ & \\
\hline Slovak Republic & HIC & $5,447,011$ & $\$$ & $19,442.71$ & $\$$ & $1,178.74$ & $\$$ & $1,388.90$ & $\$$ & 941.10 \\
\hline Slovenia & HIC & $2,067,372$ & $\$$ & $26,123.97$ & $\$$ & $1,834.16$ & $\$$ & $2,054.30$ & $\$$ & $1,325.87$ \\
\hline Solomon Islands & LMIC & 652,858 & $\$$ & $2,137.69$ & $\$$ & 106.34 & $\$$ & 111.23 & $\$$ & 73.62 \\
\hline Somalia & LIC & $15,008,154$ & $\$$ & 314.54 & $\$$ & 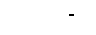 & $\$$ & - & $\$$ & - \\
\hline South Africa & UMIC & $57,779,622$ & $\$$ & $6,374.03$ & $\$$ & 428.18 & $\$$ & 461.36 & $\$$ & 230.06 \\
\hline South Sudan & LIC & $10,975,920$ & $\$$ & $1,119.65$ & $\$$ & & $\$$ & - & $\$$ & \\
\hline Spain & HIC & $46,723,749$ & $\$$ & $30,370.89$ & $\$$ & $2,389.89$ & $\$$ & $2,959.31$ & $\$$ & $1,702.51$ \\
\hline Sri Lanka & UMIC & $21,670,000$ & $\$$ & $4,102.48$ & $\$$ & 153.10 & $\$$ & 229.83 & $\$$ & 65.97 \\
\hline St. Kitts and Nevis & HIC & 52,441 & $\$$ & $19,275.42$ & $\$$ & 930.77 & $\$$ & $1,410.18$ & $\$$ & 401.81 \\
\hline
\end{tabular}


medRxiv preprint doi: https://doi.org/10.1101/2020.08.23.20180299; this version posted August 25, 2020. The copyright holder for this preprint (which was not certified by peer review) is the author/funder, who has granted medRxiv a license to display the preprint in perpetuity.

It is made available under a CC-BY-NC-ND 4.0 International license .

\begin{tabular}{|c|c|c|c|c|c|c|c|c|c|c|}
\hline St. Lucia & UMIC & 181,889 & $\$$ & $10,566.05$ & $\$$ & 489.76 & $\$$ & 728.28 & $\$$ & 205.70 \\
\hline St. Martin (French part) & HIC & 37,264 & $\$$ & - & $\$$ & 66.14 & $\$$ & 66.14 & $\$$ & 29.95 \\
\hline St. Vincent and the Grenadines & UMIC & 110,210 & $\$$ & $7,361.40$ & $\$$ & 250.22 & $\$$ & 301.51 & $\$$ & 192.06 \\
\hline Sudan & LMIC & $41,801,533$ & $\$$ & 977.27 & $\$$ & 152.02 & $\$$ & 264.36 & $\$$ & 29.62 \\
\hline Suriname & UMIC & 575,991 & $\$$ & $6,234.04$ & $\$$ & 356.05 & $\$$ & 433.74 & $\$$ & 246.99 \\
\hline Sweden & HIC & $10,183,175$ & $\$$ & $54,608.36$ & $\$$ & $5,710.59$ & $\$$ & $6,581.11$ & $\$$ & $4,768.65$ \\
\hline Switzerland & HIC & $8,516,543$ & $\$$ & $82,796.55$ & $\$$ & $9,835.96$ & $\$$ & $12,743.65$ & $\$$ & $6,174.88$ \\
\hline Syrian Arab Republic & LIC & $16,906,283$ & $\$$ & $2,032.62$ & $\$$ & - & $\$$ & 35.51 & $\$$ & - \\
\hline Tajikistan & LIC & $9,100,837$ & $\$$ & 826.62 & $\$$ & 55.70 & $\$$ & 92.49 & $\$$ & 15.93 \\
\hline Tanzania & LIC & $56,318,348$ & $\$$ & $1,050.68$ & $\$$ & 35.50 & $\$$ & 43.27 & $\$$ & 14.42 \\
\hline Thailand & UMIC & $69,428,524$ & $\$$ & $7,273.56$ & $\$$ & 221.92 & $\$$ & 248.80 & $\$$ & 173.40 \\
\hline Timor-Leste & LMIC & $1,267,972$ & $\$$ & $2,035.53$ & $\$$ & 79.89 & $\$$ & 86.99 & $\$$ & 44.55 \\
\hline Togo & LIC & $7,889,094$ & $\$$ & 679.26 & $\$$ & 38.77 & $\$$ & 58.32 & $\$$ & 7.77 \\
\hline Tonga & UMIC & 103,197 & $\$$ & $4,364.02$ & $\$$ & 203.15 & $\$$ & 225.45 & $\$$ & 133.87 \\
\hline Trinidad and Tobago & HIC & $1,389,858$ & $\$$ & $17,129.91$ & $\$$ & $1,063.83$ & $\$$ & $1,490.22$ & $\$$ & 561.62 \\
\hline Tunisia & LMIC & $11,565,204$ & $\$$ & $3,447.51$ & $\$$ & 256.50 & $\$$ & 358.85 & $\$$ & 145.27 \\
\hline Turkey & UMIC & $82,319,724$ & $\$$ & $9,370.18$ & $\$$ & 468.65 & $\$$ & 545.82 & $\$$ & 367.62 \\
\hline Turkmenistan & UMIC & $5,850,908$ & $\$$ & $6,966.64$ & $\$$ & 422.85 & $\$$ & 745.03 & $\$$ & 78.40 \\
\hline Turks and Caicos Islands & HIC & 37,665 & $\$$ & $27,142.23$ & $\$$ & $1,578.44$ & $\$$ & $1,578.44$ & $\$$ & 601.94 \\
\hline Tuvalu & UMIC & 11,508 & $\$$ & $3,700.71$ & $\$$ & 507.35 & $\$$ & 510.73 & $\$$ & 428.80 \\
\hline Uganda & LIC & $42,723,139$ & $\$$ & 642.78 & $\$$ & 37.61 & $\$$ & 52.77 & $\$$ & 6.23 \\
\hline Ukraine & LMIC & $44,622,516$ & $\$$ & $3,095.17$ & $\$$ & 141.19 & $\$$ & 217.91 & $\$$ & 59.88 \\
\hline United Arab Emirates & HIC & $9,630,959$ & $\$$ & $43,004.95$ & $\$$ & $1,323.12$ & $\$$ & $1,568.76$ & $\$$ & 958.96 \\
\hline United Kingdom & HIC & $66,488,991$ & $\$$ & $42,943.90$ & $\$$ & $3,958.02$ & $\$$ & $4,556.39$ & $\$$ & $3,175.33$ \\
\hline United States & HIC & $\begin{array}{r}327,167,43 \\
4\end{array}$ & $\$$ & $62,794.59$ & $\$$ & $9,869.74$ & $\$$ & $10,963.97$ & $\$$ & $8,077.93$ \\
\hline Uruguay & HIC & $3,449,299$ & $\$$ & $17,277.97$ & $\$$ & $1,379.10$ & $\$$ & $1,618.66$ & $\$$ & 988.73 \\
\hline Uzbekistan & LMIC & $32,955,400$ & $\$$ & $1,532.37$ & $\$$ & 135.11 & $\$$ & 205.70 & $\$$ & 62.32 \\
\hline Vanuatu & LMIC & 292,680 & $\$$ & $3,123.89$ & $\$$ & 109.81 & $\$$ & 119.06 & $\$$ & 59.16 \\
\hline Venezuela, RB & UMIC & $28,870,195$ & $\$$ & $16,054.49$ & $\$$ & & $\$$ & 445.83 & $\$$ & \\
\hline Vietnam & LMIC & $95,540,395$ & $\$$ & $2,566.60$ & $\$$ & 122.84 & $\$$ & 177.59 & $\$$ & 58.27 \\
\hline Virgin Islands (U.S.) & HIC & 106,977 & $\$$ & $35,938.02$ & $\$$ & - & $\$$ & - & $\$$ & \\
\hline West Bank and Gaza & LMIC & $4,569,087$ & $\$$ & $3,198.87$ & $\$$ & - & $\$$ & - & $\$$ & - \\
\hline Yemen, Rep. & LIC & $28,498,687$ & $\$$ & 944.41 & $\$$ & 72.04 & $\$$ & 130.36 & $\$$ & 7.34 \\
\hline Zambia & LMIC & $17,351,822$ & $\$$ & $1,539.90$ & $\$$ & 56.54 & $\$$ & 63.40 & $\$$ & 21.65 \\
\hline Zimbabwe & LMIC & $14,439,018$ & $\$$ & $2,147.00$ & $\$$ & 93.94 & $\$$ & 113.90 & $\$$ & 43.69 \\
\hline
\end{tabular}




\section{References}

1 Backer JA, Klinkenberg D, Wallinga J. Incubation period of 2019 novel coronavirus (2019$\mathrm{nCoV}$ ) infections among travellers from Wuhan, China, 20-28 January 2020. Eurosurveillance 2020; 25: 2000062. characteristics of an outbreak of 2019 novel coronavirus diseases (COVID-19) in China. Chinese J Epidemiol 2020; 41: 145-51.

3 Davies NG, Klepac $\mathrm{P}$, Liu Y, et al. Age-dependent effects in the transmission and control of COVID-19 epidemics. 2020. https://cmmid.github.io/topics/covid19/age_hypotheses.html (accessed May 4, 2020).

Prem K, Cook AR, Jit M. Projecting social contact matrices in 152 countries using contact surveys and demographic data. PLoS Comput Biol 2017; 13.

DOI:10.1371/journal.pcbi.1005697.

WorldPop, Center for International Earth Science Information Network (CIESIN). Global High Resolution Population Denominators Project. 2020.

https://www.portal.worldpop.org/demographics/ (accessed May 4, 2020).

Rees EM, Nightingale ES, Jafari Y, et al. COVID-19 length of hospital stay: a systematic review and data synthesis. medRxiv 2020; : 2020.04.30.20084780.

7 Walker P, Whittaker C, Watson O, et al. Report 12: The global impact of COVID-19 and strategies for mitigation and suppression. 2020.

https://spiral.imperial.ac.uk:8443/handle/10044/1/77735 (accessed May 4, 2020).

8 Cai Q, Huang D, Ou P, et al. COVID-19 in a designated infectious diseases hospital outside Hubei Province, China. Allergy; n/a. DOI:10.1111/all.14309.

9 Chen J, Qi T, Liu L, et al. Clinical progression of patients with COVID-19 in Shanghai, China. J Infect 2020; 80: e1-6.

10 Zhao W, Yu S, Zha X, et al. Clinical characteristics and durations of hospitalized patients with COVID-19 in Beijing: a retrospective cohort study. Infectious Diseases (except HIV/AIDS), 2020 http://medrxiv.org/lookup/doi/10.1101/2020.03.13.20035436.

11 Lei L, Jian-ya G. Clinical characteristics of 51 patients discharged from hospital with COVID-19 in Chongqing, China. medRxiv 2020; : 2020.02.20.20025536.

12 Xie H, Zhao J, Lian N, Lin S, Xie Q, Zhuo H. Clinical characteristics of non-ICU hospitalized patients with coronavirus disease 2019 and liver injury: A retrospective study. Liver Int; $\mathbf{n} / \mathbf{a}$. DOI:10.1111/liv.14449.

13 Bhatraju PK, Ghassemieh BJ, Nichols M, et al. Covid-19 in Critically III Patients in the Seattle Region - Case Series. N Engl J Med 2020; 0: null.

14 Cao B, Wang Y, Wen D, et al. A Trial of Lopinavir-Ritonavir in Adults Hospitalized with Severe Covid-19. N Engl J Med 2020; : NEJMoa2001282.

15 Linton NM, Kobayashi T, Yang Y, et al. Incubation Period and Other Epidemiological Characteristics of 2019 Novel Coronavirus Infections with Right Truncation: A Statistical Analysis of Publicly Available Case Data. J Clin Med 2020; 9. DOI:10.3390/jcm9020538. 

non-pharmaceutical interventions on COVID-19 cases, deaths and demand for hospital services in the UK: a modelling study. medRxiv 2020; : 2020.04.01.20049908.

Yang $\mathrm{X}, \mathrm{Yu} \mathrm{Y}, \mathrm{Xu}$ J, et al. Clinical course and outcomes of critically ill patients with SARS-CoV-2 pneumonia in Wuhan, China: a single-centered, retrospective, observational study. Lancet Respir Med 2020; 8: 475-81.

Wang D, Hu B, Hu C, et al. Clinical Characteristics of 138 Hospitalized Patients With 2019 Novel Coronavirus-Infected Pneumonia in Wuhan, China. JAMA 2020; 323: 1061. diagnosis in South Africa: a real-world cost analysis and economic evaluation. Lancet Glob Heal 2017; 5: e710-9.

Khan BA, Shakeel N, Siddiqui E uddin, et al. Impact of delay in admission on the outcome of critically ill patients presenting to the emergency department of a tertiary care hospital from low income country. J Pak Med Assoc 2016; 66: 509-16.

21 Sweeney S, Vassall A, Guinness L, et al. Examining Approaches to Estimate the Prevalence of Catastrophic Costs Due to Tuberculosis from Small-Scale Studies in South Africa.

Pharmacoeconomics 2020. DOI:10.1007/s40273-020-00898-3.

22 Organization IL. Labour force participation rate by sex and age. 2020.

https://www.ilo.org/shinyapps/bulkexplorer40/?lang=en\&segment=indicator\&id=EAP_2WAP _SEX_AGE_RT_A.

23 United Nations Population Division D of E and SA. World Population Prospects: the 2017 Revision. 2017. https://population.un.org/wpp/.

24 Mauch V, Bonsu F, Gyapong M, et al. Free tuberculosis diagnosis and treatment are not enough: patient cost evidence from three continents. Int J Tuberc Lung Dis 2013; 17: 381-7.

25 Ukwaja KN, Alobu I, Igwenyi C, Hopewell PC. The High Cost of Free Tuberculosis Services: Patient and Household Costs Associated with Tuberculosis Care in Ebonyi State, Nigeria. PLoS One 2013; 8: e73134.

26 Chimbindi N, Bor J, Newell M-L, et al. Time and Money: The True Costs of Health Care Utilization for Patients Receiving \&quot;Free\&quot; HIV/Tuberculosis Care and Treatment in Rural KwaZulu-Natal. J Acquir Immune Defic Syndr 2015; 70: e52-60.

Government of India, Ministry of Statistics \& Programme Implementation, National Statistics Office. Key Indicators of Social Consumption in India: Health. NSS 75th round (July 2017 - June 2018). 2019.

http://www.mospi.gov.in/sites/default/files/publication_reports/KI_Health_75th_Final.pdf (accessed May 4, 2020).

Dercon S, De Weerdt J, Bold T, Pankhurst A. Group-based funeral insurance in Ethiopia and Tanzania. World Dev 2006; 34: 685-703.

29 Council NR. Aging in Asia: Findings from New and Emerging Data Initiatives. 2012 https://www.nap.edu/catalog/13361/aging-in-asia-findings-from-new-and-emerging-datainitiatives.

30 Collins DL, Leibbrandt M. The financial impact of HIV/AIDS on poor households in South Africa. AIDS 2007; 21: S75.

31 Barasa E, Ouma PO, Okiro EA. Assessing the Hospital Surge Capacity of the Kenyan Health 
medRxiv preprint doi: https://doi.org/10.1101/2020.08.23.20180299; this version posted August 25, 2020. The copyright holder for this preprint (which was not certified by peer review) is the author/funder, who has granted medRxiv a license to display the preprint in perpetuity.

It is made available under a CC-BY-NC-ND 4.0 International license.

System in the Face of the COVID-19 Pandemic | medRxiv.

https://www.medrxiv.org/content/10.1101/2020.04.08.20057984v1.

32 Kapoor G, Sriram A, Joshi J, Nandi A, Laxminarayan R. COVID-19 in India?]: State-wise estimates of current hospital beds, intensive care unit (ICU) beds and ventilators. 2020; : 12.

33 Phua J, Faruq MO, Kulkarni AP, et al. Critical Care Bed Capacity in Asian Countries and Regions: Crit Care Med 2020; 48: 654-62.

34 Brand SPC, Aziza R, Kombe IK, et al. Forecasting the scale of the COVID-19 epidemic in Kenya. medRxiv 2020; : 2020.04.09.20059865.

35 Murthy S, Leligdowicz A, Adhikari NKJ. Intensive Care Unit Capacity in Low-Income Countries: A Systematic Review. PLoS One 2015; 10: e0116949.

36 Naidoo K, Singh J, Lalloo U. A critical analysis of ICU/HC beds in South Africa: 2008-2009. South African Med J 2013; 103: 751.

37 Bozzani FM, Mudzengi D, Sumner T, et al. Empirical estimation of resource constraints for use in model-based economic evaluation: an example of TB services in South Africa. Cost Eff Resour Alloc 2018; 16: 27.

38 Bane A, Bayisa T, Adamu F, Abdissa SG. Medical Admissions and Outcomes at Saint Paul's Hospital, Addis Ababa, Ethiopia: a retrospective study. Ethiop J Heal Dev 2016; 30: 50-6.

39 Sultan M, Mengistu G, Debebe F, Azazh A, Trehan I. The burden on emergency centres to provide care for critically ill patients in Addis Ababa, Ethiopia. African J Emerg Med Rev africaine la Med d'urgence 2018; 8: 150-4. 